\title{
Shape-controlled metal nanoparticles for electrocatalytic applications
}

\author{
${ }^{1}$ Institute of Electrochemistry, University of Alicante, Apdo. 99, Alicante E-03080, Spain, E-mail: jose.solla@ua.es
}

\begin{abstract}
:
The application of shape-controlled metal nanoparticles is profoundly impacting the field of electrocatalysis. On the one hand, their use has remarkably enhanced the electrocatalytic activity of many different reactions of interest. On the other hand, their usage is deeply contributing to a correct understanding of the correlations between shape/surface structure and electrochemical reactivity at the nanoscale. However, from the point of view of an electrochemist, there are a number of questions that must be fully satisfied before the evaluation of the shaped metal nanoparticles as electrocatalysts including (i) surface cleaning, (ii) surface structure characterization, and (iii) correlations between particle shape and surface structure. In this chapter, we will cover all these aspects. Initially, we will collect and discuss about the different practical protocols and procedures for obtaining clean shaped metal nanoparticles. This is an indispensable requirement for the establishment of correct correlations between shape/surface structure and electrochemical reactivity. Next, we will also report how some easy-to-do electrochemical experiments including their subsequent analyses can enormously contribute to a detailed characterization of the surface structure of the shaped metal nanoparticles. At this point, we will remark that the key point determining the resulting electrocatalytic activity is the surface structure of the nanoparticles (obviously, the atomic composition is also extremely relevant) but not the particle shape. Finally, we will summarize some of the most significant advances/results on the use of these shaped metal nanoparticles in electrocatalysis covering a wide range of electrocatalytic reactions including fuel cell-related reactions (electrooxidation of formic acid, methanol and ethanol and oxygen reduction) and also $\mathrm{CO}_{2}$ electroreduction.
\end{abstract}

Graphical Abstract:

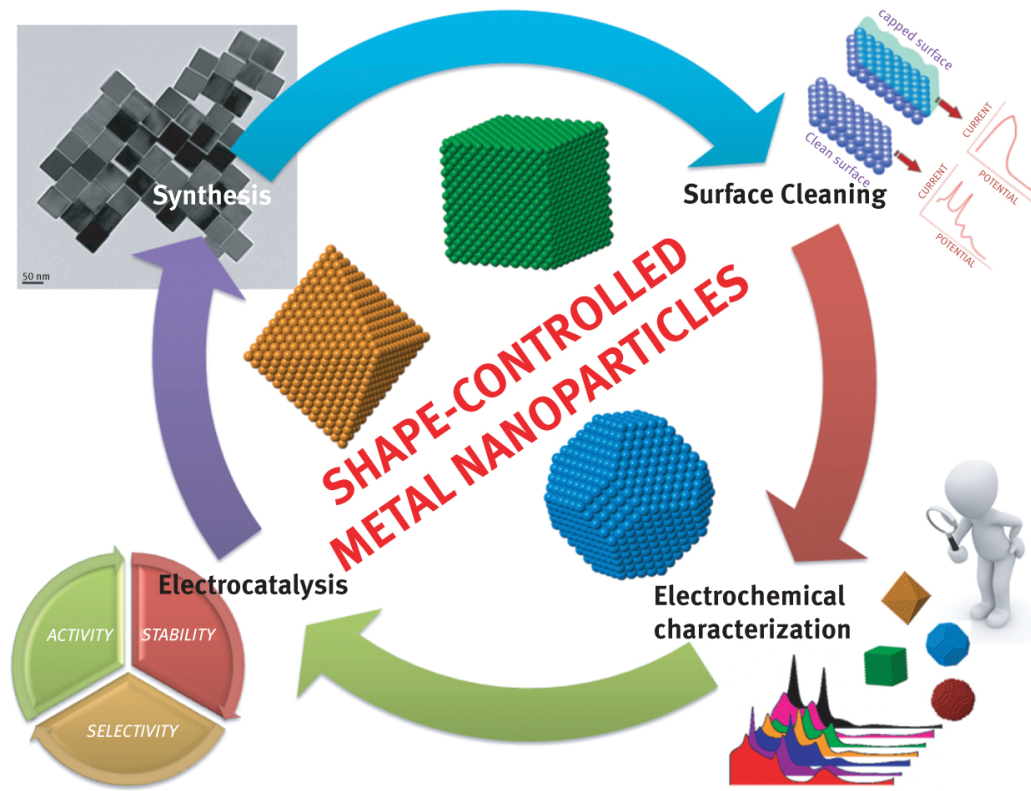

Keywords: silver, nanoparticle, biofouling

DOI: $10.1515 /$ psr-2017-0124 


\section{Introduction}

Based on the extensive and intensive knowledge gained with the use of metal single-crystal electrodes in electrocatalysis, it is now widely accepted that the surface structure, that is, the arrangement of the atoms at the surface, is one of the most relevant parameter determining the electrocatalytic properties of the material under study [1-3]. Consequently, all this knowledge must serve as a guide for the understanding and evaluation of the effect of the surface structure on the electrocatalytic properties of metal nanoparticles [4-7]. Particularly, this previous knowledge must be used to learn the methods, protocols, precautions, and requirements to be fulfilled to properly study the surface structure-reactivity correlations on the nanoscale materials.

With the aim of understanding the effect of the surface structure on metal nanoparticles, the availability of shape-controlled metal nanoparticles is extremely useful because by controlling the shape of a nanoparticle, it is possible to provide metal nanoparticles with a well-defined surface atomic arrangement and coordination, that is, nanoparticles having a well-defined surface structure. In this regard, Sun and co-workers [8,9], based on a previous contribution [10], illustrated the correlation between surface structure and particle shape by using the stereographic triangle of a face-centred cubic (fcc) metal (Figure 1). In this way, by simple analogy with the unit stereographic triangle, the low index or basal planes, located at the three vertices of the triangle, would be represented by polyhedral nanocrystals/nanoparticles bounded by the corresponding basal facets, i. e. a cube, an octahedron, and a rhombic dodecahedron corresponding with the $\{100\},\{111\}$, and $\{110\}$ basal planes, respectively. On the other hand, the polyhedra lying on the three sidelines (crystallographic zones) and inside of the triangle are polyhedra-containing stepped surfaces, that is, surfaces having a terrace-step structure. These polyhedra are also called high-index faceted nanoparticles, that is, nanoparticles containing surfaces having a set of Miller indices $\{\mathrm{hkl}\}$ with at least one index being larger than 1 ( $\{\mathrm{hk} 0\},\{\mathrm{hkk}\},\{\mathrm{hhl}\}$, and $\{\mathrm{hkl}\}(\mathrm{h}>\mathrm{k}>1 \geq$ 1)) and with a surface coordination number being generally equal to or smaller than 7 (the low-index planes have a coordination number of 9,8 , and 7 corresponding with the \{111), \{100), and (110) surfaces, respectively). These high-index-faceted nanoparticles are also known as nanoparticles with high-energy surfaces since the surface energy $(\gamma)$ associated with the different crystallographic planes increases in the order $\gamma\{111\}<\gamma\{100\}$ $<\gamma\{110\}<\gamma\{$ hkl $\}$. Finally, the nanoparticles inside the triangle are characterized by surfaces having kink sites, which induces intrinsic chirality properties.

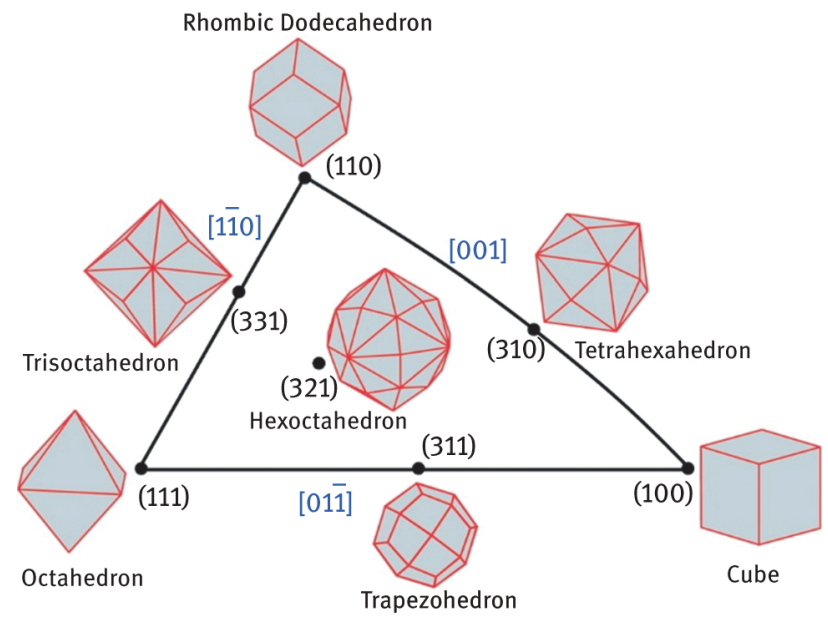

Figure 1: Unit stereographic triangle containing polyhedral nanocrystals with different surface planes. Reproduced from Ref. [9] with permission from The Royal Society of Chemistry.

It is worth noting that all these polyhedra are convex ones. In contrast, concave polyhedra are also possible by "pushing in" the centres of the corresponding facets/surfaces. However, it should be mention that the surfaces of these polyhedra are exclusively composed of similar high-index facets regardless of the curvature of polyhedra (convex or concave). To complete this collection of morphologies, it is important to include the so-called excavated and also twinned polyhedral structures.

Obviously, the stereographic triangle of polyhedral shapes shown in Figure 1 is exclusively based on geometric aspects. By incorporating thermodynamic arguments, and considering the $\gamma$ of the different crystallographic planes, a metal nanoparticle would tend to have a shape in which the total surface energy is minimized. From this energetic point of view, a shape exclusively composed by $\{111\}$ facet, that is, an octahedron, would be the most favourable one. However, this shape has a larger surface area than a cube of the same volume. Consequently, the Wulff analysis predicts a shape enclosed by a mixture of $\{111\}$ and $\{100\}$ facets. In fact, the most energetically stable shape would be a truncated octahedron with an optimal truncation fulfilling the condition 
of $\gamma(100) / \gamma(111)=d(100) / d(111)$, where $d$ represents the distance of the facets from the centre of the particle [11]. In addition, kinetic factors also play a key role and, in general, the resulting shape will determine by the relative growth rates along the $\langle 100\rangle$ and $\langle 111\rangle$ directions. The ratio of these two growth rates is generally defined as $R$ and changes from 0.58 to 0.87 and eventually to 1.73 for a cube, cuboctahedron, and octahedron, respectively. Fortunately, this energetic (thermodynamic) and/or dynamic (kinetic) control can be overcome by controlling a number of experimental parameters such as (i) the use of capping agents (to conveniently modify the surface energies and inducing the growth in a specific direction), (ii) reaction temperature, (iii) nature and concentration of reducing agents, (iv) presence of additives, and (v) metal salt precursors, among many others, to prepare nanoparticles with tuned morphologies/shapes.

On the other hand, it is worth noting that these correlations between surface structure and particle shape are based on ideal polyhedral crystals, that is, polyhedra containing perfect surface facets which is, indeed, a nonrealistic situation. In fact, the real surface of a metal nanoparticle, even a very well-defined one (in terms of size and shape), is extremely complex and its surface structure will not only be constituted by well-ordered surface facets of different dimensions but also by a determined amount of surface imperfections (defect, corner, edge, step, and kink sites), all of them contributing in a different extension (depending on the reaction under study) to the electrocatalytic activity. This aspect points out the outstanding important of having tools to characterize in detail the surface structure of a nanoparticle to then establish the correlations between its surface structure and its electrocatalytic activity. At this respect, a forthcoming section of this chapter will be devoted to the application of easy electrochemical experiments to gain both qualitative and quantitative information about the surface structure of the different metal nanoparticles and particularly shaped ones. At this point, it is again relevant to recall that, from an electrocatalytic point of view, the shape of a nanoparticle is not the key point but its surface structure.

With all these previous questions in mind, this chapter will be focused on the application of shape-controlled metal nanoparticles in electrocatalysis but exclusively from a purely electrochemical point of view. From this electrochemical perspective, a first section will be dedicated to one of the most critical requirements to perform any electrochemical analysis, the surface cleaning. As previously stated, the use of capping or additive agents is a standard way of preparing shape-controlled metal nanoparticles. However, the presence of these surface-regulating agents at the surface of the nanoparticles hinders its direct application in electrocatalysis. Therefore, once the nanoparticles are synthetized, it is mandatory to apply specific decontamination protocols to completely remove (without altering the initial surface structure of the nanoparticles) the presence, even in residual amounts, of these agents at the surface of the nanoparticles. This is not a trivial step, and, from our point of view, this aspect has been underestimated in the extensive literature related to the electrocatalytic properties of shape-controlled metal nanoparticles. Clearly, this is an innovative section in comparison with other contributions made by different groups and dealing with the electrocatalysis on shaped metal nanoparticles. Furthermore, an important section will cover the use of different electrochemical approaches to characterize the surface structure of distinct types of nanoparticles. Interestingly, this in situ surface structure characterization is not only statistically representative (thousands of nanoparticles are simultaneously measured) but also performed under similar conditions than those that will be used in the electrochemical reaction. It is worth noting that the existing literature reviewing the catalytic or electrocatalytic properties of different shaped metal nanoparticle consider the particle shape as the key point determining the resulting activity. However, from an electrochemical point of view, it is well-recognized that it is the surface structure the crucial parameter controlling the electrochemical reactivity. Consequently, the description of available electrochemical tools to characterize in detail the surface structure of these shaped metal nanoparticles is an innovative approach in comparison with previous contributions. To conclude this chapter, a last section containing some of the most significant advances/results on the use of these shaped metal nanoparticles in electrocatalysis will be included. This section will not only cover relevant reactions for low-temperature fuel cells such as formic acid, methanol, and ethanol electrooxidation or oxygen reduction but also other interesting reactions such as $\mathrm{CO}_{2}$ electroreduction.

\section{Preparation methods}

\subsection{Synthesis of shape-controlled metal nanoparticles}

To the best of our knowledge, the first examples of preparation of shape-controlled nanoparticles were achieved by using gas chemisorption. In 1985, Wang et al. observed that the shape of Pt nanocrystals (supported on an amorphous $\mathrm{SiO}_{2}$ substrate) was modified in the presence of different gas molecules at $600^{\circ} \mathrm{C}$ for $24 \mathrm{~h} \mathrm{[12]}$. Under these conditions, Pt nanocrystals with a truncated cuboctahedral and cubic shape were obtained when samples were annealed in $\mathrm{N}_{2}$ and $\mathrm{H}_{2}$, respectively. Similarly, Harris in 1986 observed that cubic Pt nanocrystals 
(supported on $\mathrm{Al}_{2} \mathrm{O}_{3}$ ) were obtained when a $\mathrm{H}_{2}$ atmosphere containing a trace amount of $\mathrm{H}_{2} \mathrm{~S}$ at $500{ }^{\circ} \mathrm{C}$ for 16 $\mathrm{h}$ was used [13]. These two examples clearly showed that by using some gaseous species able to be adsorbed in a specific type of facet, the shape of a metal nanocrystal supported on a solid substrate can be effectively modified.

Going from this gas-phase method to a solution-phase one, El-Sayed and co-workers in 1996 reported the first example of shape-controlled synthesis of metal nanocrystals in water solution by preparing cubic and tetrahedral $\mathrm{Pt}$ nanoparticles through a chemical reduction of $\mathrm{K}_{2} \mathrm{PtCl}_{4}$ by $\mathrm{H}_{2}$ in the presence of sodium polyacrylate $(\mathrm{NaPa})$ [14]. Obviously, the employ of solution-phase methods for the synthesis of shape-controlled metal nanoparticles seems more convenient than the gas-phase ones where limited option of gases, as well as the use of elevated temperatures, is required. In contrast, in solution phase, there is not only a great availability of capping agents that can be used for each type of facet, but also the experimental conditions are mild.

From these pioneer contributions and due to the enormous efforts coming from many research groups, the existing literature contains now an incredible number of methodologies for the preparation of shape-controlled metal nanoparticles. In this chapter, we are not going to review the innumerable methodologies for the preparation of shape-controlled metal nanoparticles and the readers interested in this topic are referred to some of the relevant and excellent reviews exclusively focused on synthetic aspects [15-28].

Among all these different methods, the use of colloidal routes is one of the most employed. This method is essentially based on the chemical reduction of a metallic precursor in the presence of a capping agent. The role of this capping agent is extremely important in the process because during the growth step, it can selectively bind to a specific facet on the nanoparticles, thus altering their surface energies and promoting the formation of the nanoparticles with a preferential shape that maximizes the expression of that type of facet. Additionally, the effect of the capping can be also explained from a kinetic perspective, that is, taking into consideration the relative growth rates of different crystallographic structures. When the capping is adsorbed in a facet, the subsequent deposition of new atoms onto this facet will be importantly reduced and, therefore, that facet will have a slower growth rate. Consequently, the resulting nanoparticles will have a preferential shape in which that facet will be preferentially expressed. Xia et al. [23] schematically illustrated the role of two complementary capping agents, one for $\{100\}$ facets and the other for $\{111\}$ facets during the growth of a truncated octahedral seed, Figure 2.

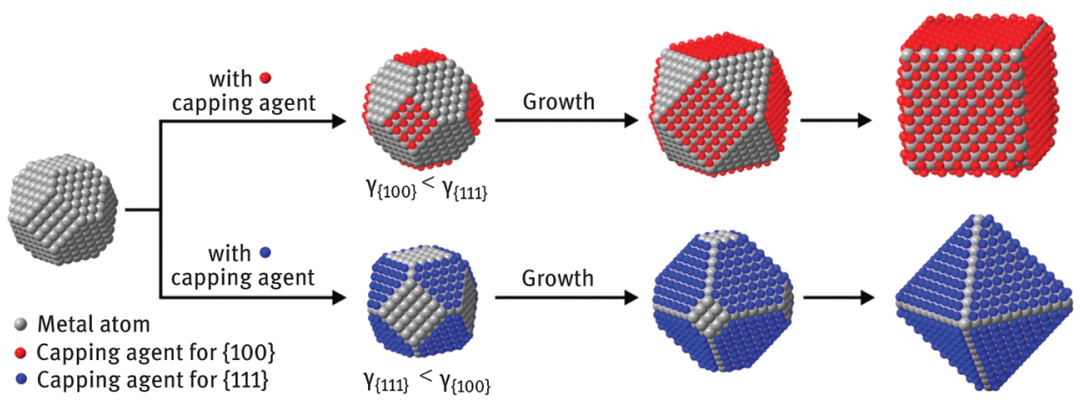

Figure 2: Schematic illustration explaining the role of capping agents during the growth of a single-crystal truncated octahedral seed of an fcc metal. Reprinted with permission from Ref [23]. Copyright (2015) American Chemical Society.

As shown in Figure 2, the resulting shape is determined by the capping agent used. Thus, a cubic crystal is obtained when a capping agent with a preference for the $\{100\}$ facets is employed. Contrarily, when a capping agent with a preference for the $\{111\}$ facets is introduced, a crystal with an octahedral shape is achieved. Under this thermodynamic control, different nanoparticles with well-controlled shapes can be obtained. However, under realistic condition, the capping agent is not the only parameter determining the resulting shape but also other experimental parameters including temperature, electrolyte, reducing time, nature of the reducing agent, and some others will play a significant role in defining the final shape obtained which will be ultimately determined by both thermodynamic and kinetic control [23].

Interestingly, it is also worth noting that some shape-controlled metal nanoparticles can also be prepared using capping-free methodologies. In this regard, the electrochemical approach initially developed by Sun and co-workers is particularly interesting [8,9,29-31]. Other interesting approaches are capping-free solvothermal synthesis [32-35], cathodic corrosion [36], or solid-state chemistry methods [37, 38].

\subsection{Surface cleanness: a key point in electrocatalysis}

It is well-documented that the electrocatalysis on metal single crystals is profoundly marked by the publication in 1980 of the flame annealing treatment by Clavilier et al. [39] This work represents the starting point from 
which it was possible to correctly understand the relationships between surface structure and electrocatalytic surface reactivity. Very briefly, this simple and elegant cleaning treatment allows obtaining clean and wellordered Pt single-crystal surfaces to be used in electrochemical measurements in a reproducible way [40]. For instance, this worldwide adapted treatment allowed correlating the voltammetric profile in the so-called hydrogen adsorption/desorption region of any Pt single-crystal electrode including basal, stepped, and kinked surfaces with their intrinsic surface structure (surface orientation, ordered domains, and population of step and terrace sites). Consequently, to correctly understand the correlations between particles shape, surface structure, and electrocatalytic properties with the shaped metal nanoparticles, this cleaning requirement must be fully satisfied.

This cleaning requirement is obviously faced with the employ of capping or surface-stabilizing agents which are fundamental to obtain shaped nanoparticles. Therefore, once shaped metal nanoparticles are synthesized, these surface-regulating agents must be completely removed from the surface of the shaped nanoparticles [41]. Consequently, the development of effective surface cleaning methodologies capable of removing such capping or surface-regulating agents from the surface of the corresponding nanoparticles becomes an extremely important prerequisite to subsequently evaluate their electrocatalytic properties for any reaction of interest. If the cleaning is not properly performed, the electrocatalytic activity of the nanoparticles will be affected in a noncontrolled way, and non-reproducible and non-comparable results will be obtained. Figure 3 schematically represents the effect of an incomplete removal of the capping or surface-regulating agents from the surface of the shaped nanoparticles on their electrocatalytic properties. In addition, there are some important aspects to mention. The first one deals with the wide variety of capping and/or surface-regulating agents that have been used for the synthesis of shaped nanoparticles. This fact implies that a "common" cleaning methodology is not available, and a specific decontamination must be developed, optimized, and tested for each capping agent. In this way, each specific cleaning will basically depend on two main parameters such as (i) the chemical nature of the capping agent and (ii) its interaction with the surface of the shaped nanoparticles, the latter being also affected by the nature of the metal as well as by their surface structure. On the other hand, it is worth noting that this decontamination method must be able to remove the capping agents but without affecting the intrinsic surface structure of the nanoparticles. This point will be exemplified and discussed in a forthcoming section.

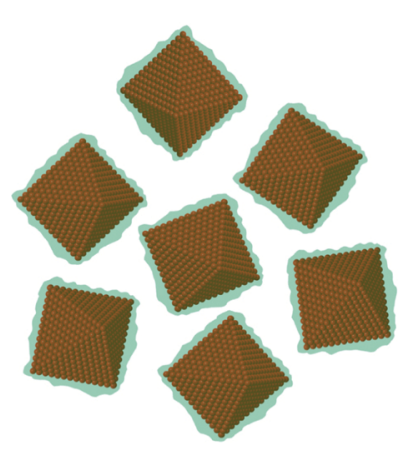

As-prepared shaped metal nanoparticles

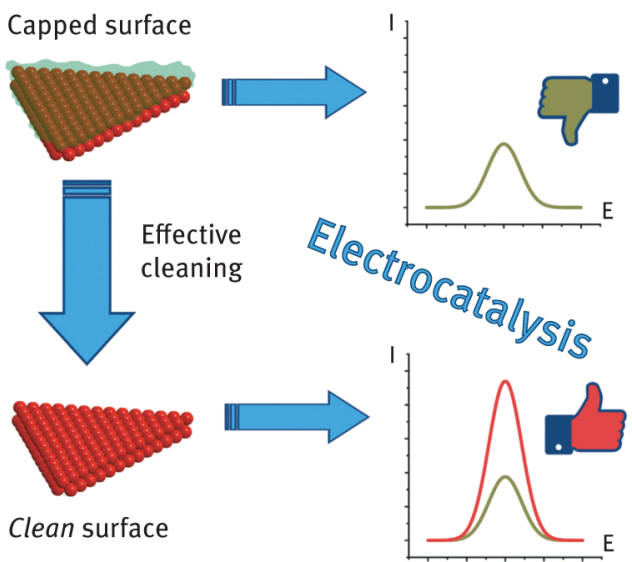

Figure 3: Schematically representation of the importance of having clean shaped metal nanoparticles. Reprinted from Ref. [41], Copyright (2017) with permission from Elsevier.

\subsubsection{Electrochemical probes for surface cleanness evaluation on shaped metal nanoparticles}

Once discussed about the mandatory requirement of having shaped nanoparticles with clean surfaces to properly study their electrochemical properties, the question now is how to evaluate this surface cleanness. Fortunately, and again from previous knowledge of surface electrochemistry on metal single crystals, it is possible to perform some electrochemical analysis to evaluate this surface cleanness. For Pt and Pd surfaces, it is widely accepted that the so-called hydrogen region (involving the hydrogen and anion adsorption-desorption states) obtained in $0.5 \mathrm{M} \mathrm{H}_{2} \mathrm{SO}_{4}$ solution is a very sensitive process for the evaluation of the level of cleanness of the surface [2, 3, 40, 42-45]. Very briefly, if the voltammetric profile displays well-defined contributions, in terms of sharpness and reversibility (between desorption vs. adsorption states), that is an indisputable proof of the adequate cleanness of the surface. To illustrate that, Figure 4 shows the voltammetric profiles in the so-called hydrogen region obtained with (a) clean and [46] (b) contaminated [47] cubic and octahedral/tetrahedral Pt nanoparticles both obtained in $0.5 \mathrm{M} \mathrm{H}_{2} \mathrm{SO}_{4}$. The sharpness and reversibility of the adsorption desorption states 
showed with clean samples clearly contrast with the poor definition of the voltammetric features obtained with contaminated samples are evaluated.
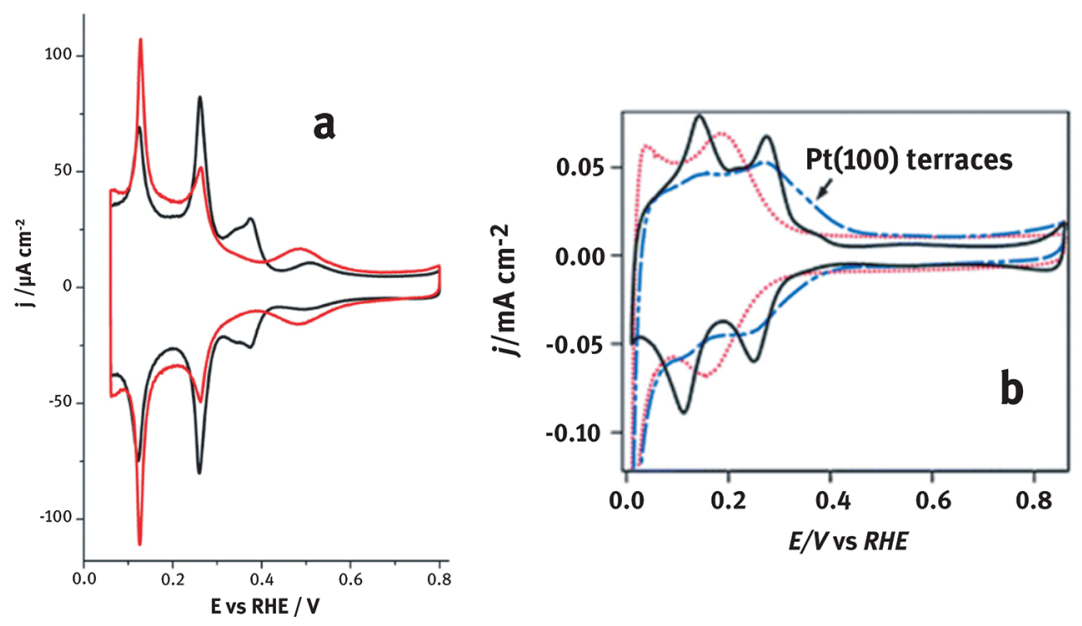

Figure 4: Cyclic voltammograms corresponding with (a) clean cubic (black line) and octahedral (red line) Pt nanoparticles (data taken from Ref. [46]) and with (b) contaminated cubic (blue dashed line) and octahedral (red dotted line) Pt nanoparticles (a commercial Pt black (black solid line) is also included) obtained in $0.5 \mathrm{M} \mathrm{H}_{2} \mathrm{SO}_{4}$ at $50 \mathrm{mV} \mathrm{s}{ }^{-1}$. Reproduced from Ref. [47] with permission from The Royal Society of Chemistry.

Obviously, for shape-controlled Pt and Pd-based alloy and/or core-shell nanoparticles (this is one of the hot topics in electrocatalysis), the evaluation of the surface cleanness through the so-called hydrogen region is much more complex due to the intrinsic complexity of the surfaces [48-50]. However, at least, one should verify that monometallic samples, prepared in similar conditions than those used for the synthesis of the shaped alloy and/or core-shell nanoparticles, display the voltammetric features corresponding to clean surfaces.

For Au surfaces, the situation is little bit more difficult due to the inexistence of hydrogen adsorptiondesorption features on this metal. An interesting alternative is the analysis of the voltammetric profile of the oxide region based on previous contributions by Hamelin et al. [51, 52] with Au single-crystal electrodes. This analysis has been already used with different shape-controlled Au nanoparticles containing both low- and high-index surfaces [53-55]. However, it is important to mention that for all these Au surfaces, the voltammetric profile in the oxide region as well as the charge involved in both the surface oxide process and its subsequent surface reduction should remain stable during the first cycles (about five cycles). In this sense, it is worth recalling that, at these high potential values, the organic impurities can be electrochemical oxidized, thus in situ cleaning the surface. However, this electrochemical surface oxidation/reduction cleaning also perturbs the surface structure and, as shown with gold single-crystal electrodes [56, 57], the well-defined surface structure of the Au single-crystal electrodes is clearly modified with the electrochemical oxidation/reduction cycles. In this way, despite the structural changes induced by a single-oxidation cycle with gold surfaces are remarkably smaller than those observed for Pt electrodes [58], this surface oxidation/reduction cleaning treatment should not be performed.

Also, by using different underpotential deposition (UPD) processes, some information about the surface cleanness can be also deduced. Among others, lead (Pb) UPD on Au [59-65] and copper (Cu) UPD on Pd [66-68] and $\mathrm{Pt}[69,70]$ are particularly interesting. Figure 5 displays the corresponding $\mathrm{Pb}$ UPD features obtained with clean and contaminated Au nanoparticles [63]. As previously discussed, through the analysis of the definition of the different voltammetric features, relevant information about the cleanness of the surface can be extracted. 


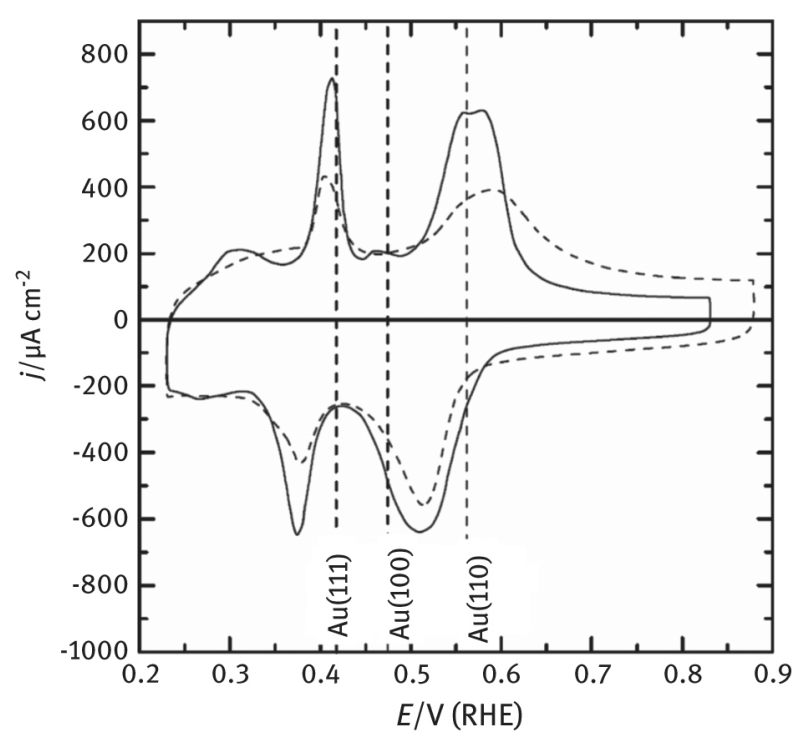

Figure 5: Voltammetric profiles corresponding with clean (solid line) and contaminated (dashed line) Au nanoparticles obtained in $0.1 \mathrm{M} \mathrm{NaOH}+10^{-3} \mathrm{M} \mathrm{Pb}\left(\mathrm{NO}_{3}\right)_{2}$ at $50 \mathrm{mV} \mathrm{s}^{-1}$. Reprinted from Ref. [63], Copyright (2014) with permission from Elsevier.

With all these previous aspects in mind, in the following section, we will summarize some of the most representative decontamination protocols that have been verified, particularly using electrochemical probes, and applied to different shape-controlled metal nanoparticles.

\subsubsection{Surface cleaning methodologies for shape-controlled metal nanoparticles}

The first examples of clean shaped metal nanoparticles were reported by our group in 2004 [71, 72]. In brief, cubic Pt nanoparticles prepared in the presence of NaPA were cleaned by direct addition of some $\mathrm{NaOH}$ pellets to the colloidal suspension. The addition of the $\mathrm{NaOH}$ induces the destabilization of the colloid and the nanoparticles precipitate. The samples are then collected and washed a couple of times with ultrapure water. The clean cubic Pt nanoparticles were finally stored in ultrapure water. The same cleaning protocol was applied later to cuboctahedral and tetrahedral-octahedral Pt nanoparticles prepared using a similar methodology (using NaPA as capping agent). The effectiveness of the cleaning was evidenced by the definition and reversibility of the cyclic voltammograms in the so-called hydrogen region obtained in $0.5 \mathrm{M} \mathrm{H}_{2} \mathrm{SO}_{4}$.

This cleaning protocol was found to be also effective with cubic $\mathrm{Pd}$ nanoparticles prepared in the presence of cetyltrimethylammonium bromide (CTAB) [73-75]. Interestingly, Coutanceau and co-workers also verified that this alkaline cleaning protocol was again effective, in this case, with cubic $\mathrm{Pt}$ nanoparticles prepared using tetradecyltrimethylammonium bromide (TTAB) as capping agent [76,77]. The electrochemical response of these TTAB-prepared cubic Pt nanoparticles in $0.5 \mathrm{M} \mathrm{H}_{2} \mathrm{SO}_{4}$ was essentially similar to that observed with the NaPAprepared cubic Pt nanoparticles [46, 78-80], thus pointing out that, independent of the synthetic methodology used, and particularly the chemical nature of the capping agent employed, similar nanoparticles must display similar voltammetric features if they are properly cleaned.

Unfortunately, this cleaning protocol did not provide satisfactory results with shaped Pt nanoparticles prepared with polyvinylpyrrolidone (PVP) [76, 81-83]. In these contributions, the absence of well-defined voltammetric features denoted the important presence of residual PVP blocking the surface sites. The PVP is one of the most usual capping agents for the preparation of shape-controlled metal nanoparticles [84] and, to the best of our knowledge, the first evidence of clean shape-controlled Pt nanoparticles prepared with PVP was published by Koper and co-workers [85] by employing a $\mathrm{H}_{2} \mathrm{O}_{2} / \mathrm{H}_{2} \mathrm{SO}_{4}$ solution to remove the PVP. They claimed that the PVP removal was physically removed from the surface of the nanoparticles by the oxygen bubbling produced during the decomposition of the $\mathrm{H}_{2} \mathrm{O}_{2}$. Subsequently, Levendorf et al. proposed a new protocol to obtain PVP-free cubic and octahedral/tetrahedral Pt nanoparticles using an adapted liquid phase UV photo-oxidation technique [86]. In this case, after different washing steps with different solvents - acetone, ethanol-hexane mixtures, and ethanol - the ethanolic suspension containing the nanoparticles was initially alkalinized. This basic solution containing the nanoparticles was UV irradiated $(254 \mathrm{~nm})$ for $1 \mathrm{~h}$, while about $1 \mathrm{~mL}$ of $\mathrm{O}_{2}$-saturated $\mathrm{H}_{2} \mathrm{O}_{2}$ solution was added to the solution every $10 \mathrm{~min}$. After that, the solution containing the nanoparticles was finally purified by repetitive centrifugation and precipitation with ethanol. The voltammetric profiles of 
these shaped Pt nanoparticles recorded in $0.5 \mathrm{M} \mathrm{H}_{2} \mathrm{SO}_{4}$ as well as TGA analyses evidenced the effectiveness of the cleaning protocol proposed.

Later, Yang et al. proposed an alternative electrochemical cleaning procedure to obtained clean shapecontrolled Pt nanoparticles prepared with PVP and also with oleylamine/oleic acid [87]. The removal of the different capping agents was achieved by cycling the samples (initially washed with a $\mathrm{NaOH}$ saturated ethanolic solution (PVP samples) or with a hexane/ethanol mixture (oleylamine/oleic acid samples), and finally redispersed in ethanol and hexane, respectively) between 0 and $1.0 \mathrm{~V}$ (vs. RHE) in $0.5 \mathrm{M} \mathrm{NaOH}$ at a scan rate of 0.5 $\mathrm{V} \mathrm{s}^{-} 1$ for at least 100 cycles. The sample was then transferred to an electrochemical cell containing $0.5 \mathrm{M} \mathrm{H}_{2} \mathrm{SO}_{4}$ where, by regarding the so-called hydrogen region, the cleanness of the surface was evaluated. The results obtained indicated that both PVP and oleylamine/oleic acid could be effectively removed from the surface of the nanoparticles.

Very interestingly, Coutanceau and co-workers $[88,89]$ reported a much easier method to clean shaped controlled Pd nanoparticles prepared with PVP. This protocol is essentially similar to the alkaline cleaning one but using a significantly higher $\mathrm{NaOH}$ concentration. Briefly, the PVP-colloidal suspension containing the shaped $\mathrm{Pd}$ nanoparticles was diluted with $\mathrm{H}_{2} \mathrm{O}$ after which $\mathrm{NaOH}$ was added until getting a $1 \mathrm{M} \mathrm{NaOH}$ solution. Under these conditions, the nanoparticles were collected by precipitation and washed with water. The voltammetric profiles obtained in $0.5 \mathrm{M} \mathrm{H}_{2} \mathrm{SO}_{4}$ displayed characteristics features of clean samples.

Neergat and co-workers reported two new cleaning protocol s for the removal of PVP and others surfaceregulating additives such as $\mathrm{Br}^{-}, \mathrm{Cl}^{-}$, and citrate ions employed during the preparation of shape-controlled $\mathrm{Pd}$ nanoparticles [90,91]. After some preliminary cleaning steps for the removal of the excess of chemicals, the samples were subsequently treated with tert-butylamine (TBA) [91] or $\mathrm{NaBH}_{4}$ [90] and finally collected by centrifugation and washed with different solvents (ethanol or water). However, and despite some improvement in terms of cleaning (about a $90 \%$ cleaning was estimated) and consequently on their electrocatalytic properties (enhanced ORR activities), the samples were not completely clean as deduced from CHN analyses.

Luo et al. employed a combined TBA/ $\mathrm{NaBH}_{4}$ cleaning to produce clean Pt-Pd nanocubes obtained in the presence of PVP [92]. The removal of the PVP was carried out by dispersing the nanoparticles in a TBA/ $\mathrm{NaBH}_{4}$ aqueous solution $\left(\mathrm{NaBH}_{4} / \mathrm{TBA} /\right.$ water, $\left.1.9 \mathrm{mg} / 20 \mathrm{~mL} / 5 \mathrm{~mL}\right)$ for $30 \mathrm{~min}$ under continuous stirring and at room temperature. The sample was then collected by centrifugation and washed with an ethanol/acetone mixture. In this case, the surface cleaning was confirmed by Raman and FTIR studies, and the electrocatalytic activity of the clean Pt-Pd nanocubes towards methanol oxidation in $0.1 \mathrm{M} \mathrm{HClO}_{4}$ displayed an evident enhancement.

Arán-Ais et al. presented a new methodology to clean various shape-controlled Pt nanoparticles prepared in oleylamine/oleic acid [93]. The different shaped Pt nanoparticles were initially washed with a hexane/ethanol mixture and then with a methanolic solution containing $\mathrm{NaOH}$. After complete precipitation of the nanoparticles, the sample was washed with acetone. This treatment was repeated at least three times, after which the sample was washed and stored in ultrapure water. Subsequently, the samples were electrochemically characterized in $0.5 \mathrm{M} \mathrm{H}_{2} \mathrm{SO}_{4}$ displaying well-defined voltammetric features. Interestingly, as shown in Figure 6, if the shaped Pt nanoparticles were not conveniently cleaned, they showed a voltammetric profile with a poor definition as a consequence of their insufficient surface cleanness.

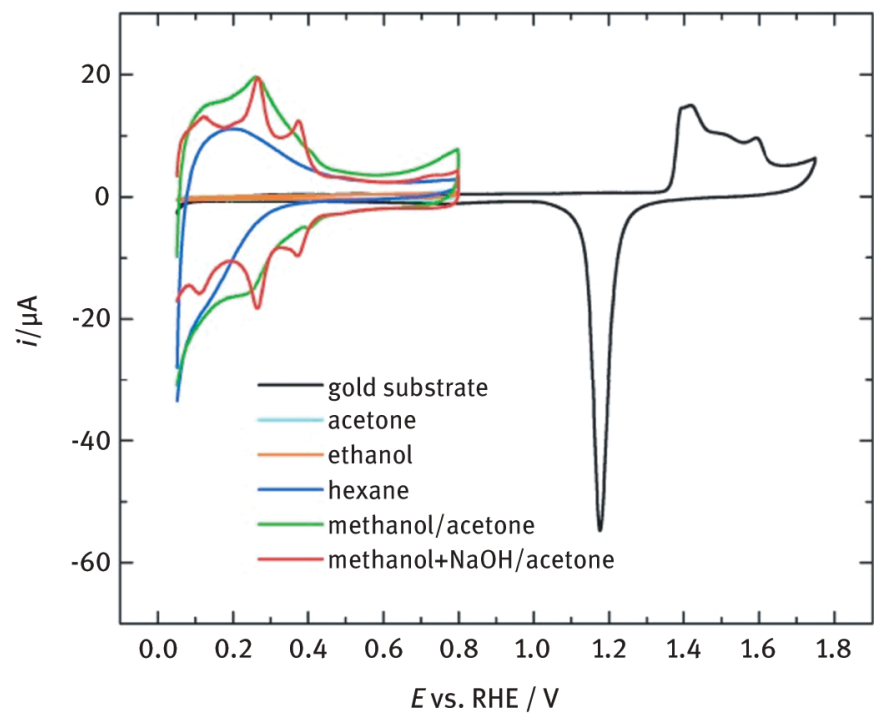

Figure 6: Voltammetric profiles obtained with cubic Pt nanoparticles after being treated with different cleaning protocols. Test solution: $0.5 \mathrm{M} \mathrm{H}_{2} \mathrm{SO}_{4}$. Sweep rate: $50 \mathrm{mV} \mathrm{s}^{-1}$. Reprinted from Ref. [93] with permission from John Wiley and Sons. 
More recently, Oezaslan et al. reported two novel approaches to clean Pt nanocubes obtained in the presence of PVP [94, 95]. In the first case [94], they proposed an electrochemical cleaning for the removal of the capping agent. Thus, after testing different supporting electrolytes $\left(\mathrm{pH} \sim 1,0.1 \mathrm{M} \mathrm{HClO}_{4}\right.$ and $\left.\mathrm{pH} \sim 13,0.1 \mathrm{M} \mathrm{NaOH}\right)$ as well as different upper potential limits $(0.8,1.0,1.2$ and $1.4 \mathrm{~V} / \mathrm{RHE})$, they stated that the electrochemical cycling (200 cycles at a scan rate of $200 \mathrm{mV} \mathrm{s}^{-1}$ ) up to $0.8 \mathrm{~V}$ in $0.1 \mathrm{M} \mathrm{HClO}_{4}$ was the most convenient one in terms of cleaning and particle shape stability. In a subsequent contribution, they incorporated an optimized washing step before electrochemical cleaning [95]. Based on FTIR results, they suggested that a washing process in methanol/ethanol (3:1) significantly removed the physisorbed PVP. After this washing step, the samples were electrochemically treated by cycling between 0.06 and $1.0 \mathrm{~V}$ vs. RHE for 200 cycles in an Ar-saturated $0.1 \mathrm{M}$ $\mathrm{HClO}_{4}$ solution.

For shape-controlled $\mathrm{Au}$ nanoparticles, the electrochemical deposition of a $\mathrm{PbO}_{2}$ film in alkaline solution $[63-65,96]$ has been shown to be a very effective cleaning protocol. In brief, the cleaning consists in cycling the gold nanoparticles in the $\mathrm{Pb}^{2+}$ containing alkaline solution from 0.25 to $1.70 \mathrm{~V}$ (vs. RHE) to form the $\mathrm{PbO}_{2}$ film. The electrocatalytic properties of the $\mathrm{PbO}_{2}$ film for the oxidation of organic species contribute to the removal of the capping agents used. Interestingly, and despite the high potential values, this process preserves the surface structure of the nanoparticles as it was demonstrated with both single-crystal and polyoriented Au electrodes [65]. This cleaning protocol has been successfully used for different shaped Au nanoparticles prepared using very different capping agents including polyethylene glycol dodecyl ether (Brij $\left.{ }^{\circledR} 30\right)$ [63], CTAB [55, 64], and PVP [97], among others. For instance, Hassel et al. applied this cleaning methodology to different Au nanoplates prepared with PVP [97]. As illustrated in Figure 7, the good definition of the voltammetric profile for the $\mathrm{Pb}$ UPD process obtained with the clean samples in comparison with the contaminated ones clearly point out the effectiveness of this cleaning process.

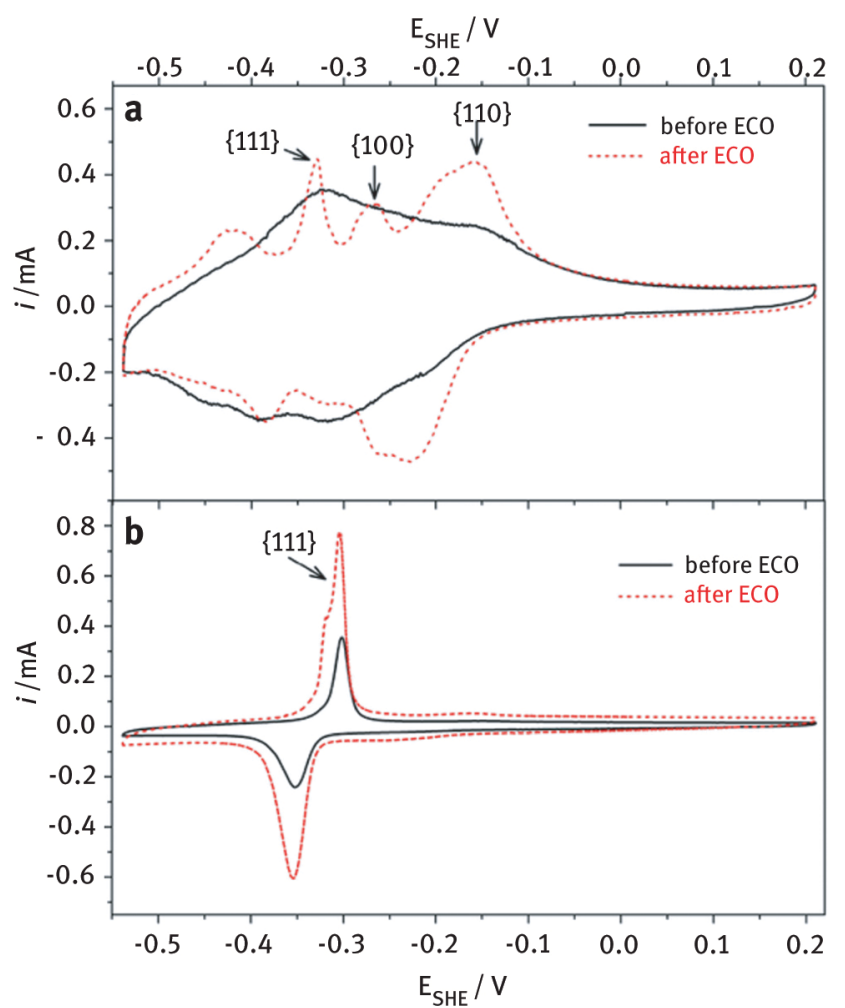

Figure 7: CV profiles of (a) $\{110\}$ and (b) $\{111\}$ Au nanoplates in $0.1 \mathrm{M} \mathrm{NaOH}+10^{-3} \mathrm{M} \mathrm{Pb}\left(\mathrm{NO}_{3}\right)_{2}$ before and after ECO. Scan rate: $20 \mathrm{mV} \mathrm{s}^{-1}$. Reprinted from Ref. [97] with permission from John Wiley and Sons.

Lee and co-workers used an $\mathrm{O}_{2}$ plasma treatment to obtain clean gold nanocrystals prepared with PVP $[54,98]$. After the cleaning, the cubic and octahedral gold nanocrystals displayed well-defined voltammetric features in the surface oxide region which were completely missing before the oxygen plasma treatment. 


\section{Characterization methodologies}

\subsection{Electrochemical probes for the characterization of the surface structure of the shaped metal nanoparticles: qualitative and quantitative approaches}

As discussed in the previous section, by using some electrochemical probes, the surface cleanness of different shaped metal nanoparticles can be easily visualized. However, these electrochemical probes can be also employed to gain relevant and very detailed information about the surface structure of the different nanoparticles. Thus, in this section, we will describe some of the most interesting electrochemical analysis to characterize the surface structure of the shaped metal nanoparticles. For Pt and Pd surfaces, it is well-recognized that the voltammetric profile in the so-called hydrogen region obtained in $0.5 \mathrm{M} \mathrm{H}_{2} \mathrm{SO}_{4}$ solution can be taken as fingerprint of their crystalline surface structure [99]. In this way, by simple inspection of the relative intensity of the different voltammetric features present in this hydrogen region, one can directly obtain qualitative information about the surface structure of the sample, that is, about the different active sites present on the surface. To illustrate the case, and focusing exclusively on Pt, Figure 8 shows the voltammograms obtained in $0.5 \mathrm{M} \mathrm{H}_{2} \mathrm{SO}_{4}$ with quasi-spherical, cubic, octahedral/tetrahedral, and truncated octahedral Pt nanoparticles [46]. First, as mentioned in the previous section, the well-resolved hydrogen peaks point out the correct surface cleanness of the shaped Pt nanoparticles. Then, it is obvious that the voltammetric responses of the samples are clearly different. As extensively described in previous contributions, and based on Pt single-crystal surfaces, the main voltammetric features are (i) the peak at $0.12 \mathrm{~V}$, which is related to $\{110\}$-type sites; (ii) the peak at $0.27 \mathrm{~V}$, which contains two contributions from (100) step sites on $\{111\}$ terraces and the sites close to the steps on the $\{100\}$ terraces; (iii) the signals at $0.35-0.37 \mathrm{~V}$ attributed to ordered $\{100\}$ terraces; and (iv) the signal at $0.5 \mathrm{~V}$, related to the ordered $\{111\}$ terraces. As expected, all these voltammetric profiles can be perfectly correlated with those reported with Pt single-crystal electrodes [99].
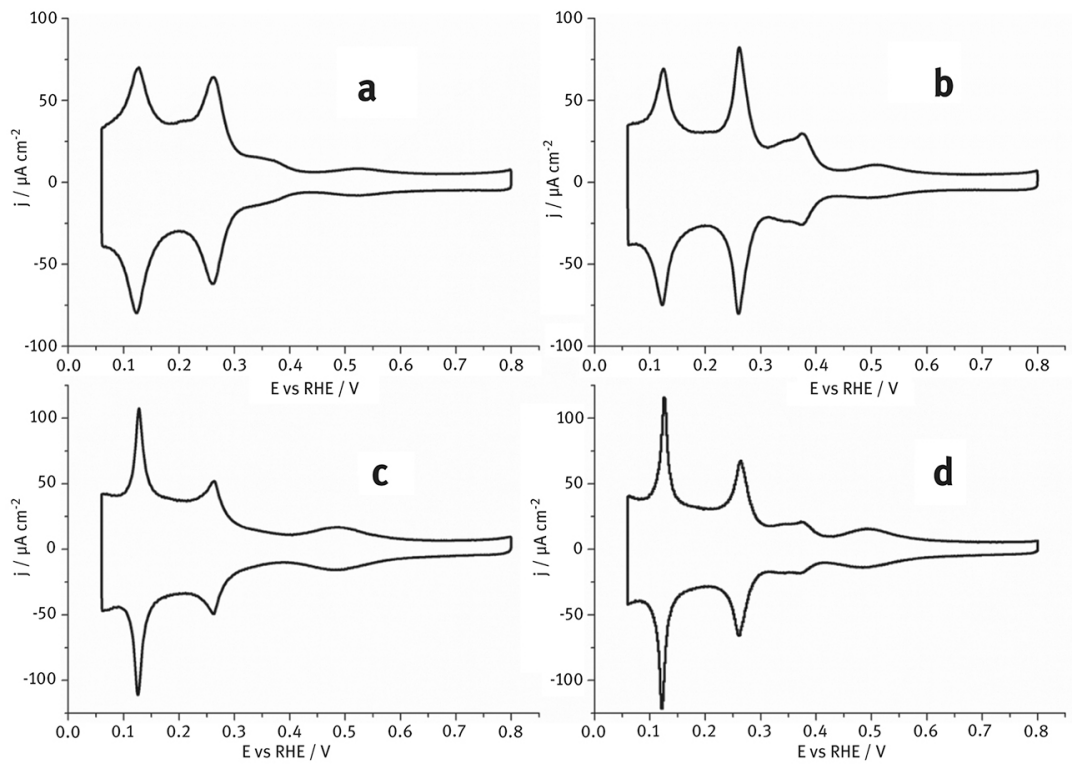

Figure 8: Representative voltammetric profiles of (a) quasi-spherical, (b) cubic, (c) octahedral/tetrahedral, and (d) truncated octahedral Pt nanoparticles obtained in different supporting electrolytes at $50 \mathrm{mV} \mathrm{s}^{-1}$. Data taken from [46].

In this way, and from the relative distribution of charge among the voltammetric peaks, it is possible to get a qualitative analysis of the relative distribution of electrochemically available Pt sites present at the whole surface of each type of sample [99, 100]. Figure 9 schematically illustrates the correspondence between the voltammetric features and the different types of active sites present at the surface of a Pt nanocube. By performing a similar analysis, but using the voltammetric profiles displayed in Figure 8, it is now consistent to correlate the particle shape with the corresponding surface structure and the quasi-spherical, cubic, octahedral/tetrahedral and truncated octahedral Pt nanoparticles present a polyoriented, (100), (111), and (100)-(111) preferentially oriented surface structure, respectively. These results are in good agreement with what it would be expected from the correlation between surface structure and particle shape by using the stereographic triangle of a fcc metal (Figure 1). However, it is also evident that the surface of all these Pt nanoparticles is very complex and it does not contain only a single type of surface sites, but all the adsorption-desorption states are observed but presenting a different contribution as a function of their particular surface structure [101]. In addition, it 
is worth noting that these surfaces always present a determined number of surface defects (corner, edge, step, and kink sites) also contributing to the resulting voltammetric profile.

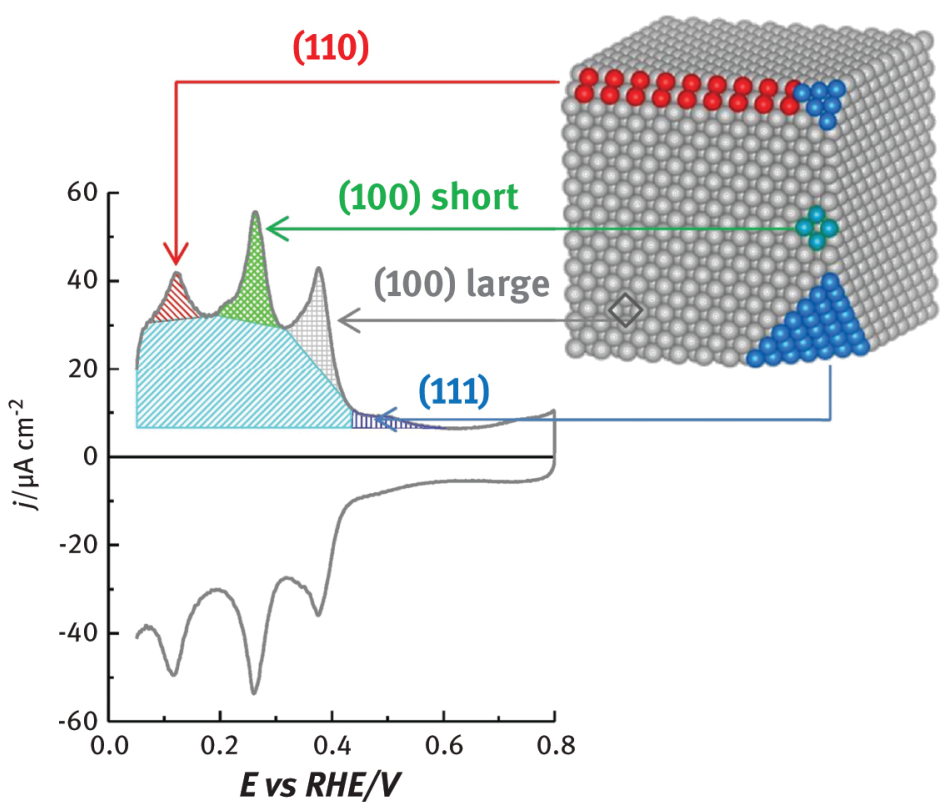

Figure 9: Characteristic voltammetric profile and atomic model of (100)-preferentially oriented Pt nanoparticles in 0.5 $\mathrm{H}_{2} \mathrm{SO}_{4}$, showing bevelled and truncated edges and its correspondence with the different voltammetric features. Reprinted from Ref. [101], Copyright (2018) with permission from Elsevier.

Analogue studies can be also performed in other supporting electrolytes $\left(0.1 \mathrm{M} \mathrm{NaOH}\right.$ and $\left.0.1 \mathrm{M} \mathrm{HClO}_{4}\right)$, although these media are less convenient for the analysis of the surface structure of the shaped Pt nanoparticles due to the overlapping of some voltammetric features [46]. Finally, it is worth mentioning that the overall charge involved in the so-called hydrogen region is also known to be proportional to the total amount of surface Pt atoms and, therefore, the integrated charge obtained from the CV can be used to properly calculate the electroactive surface area of the Pt nanoparticles. A similar analysis between the voltammetric profile in the hydrogen region obtained in different supporting electrolytes and the surfaces structure of the electrodes is also possible for Pd surfaces. Readers interested in this topic are referred to [49].

As previously stated, the surface cleaning must be performed without perturbing the surface structure of the nanoparticles. In this way and considering the high sensitivity of the hydrogen region to the surface structure, this reaction can help to verify if the cleaning protocol alters or not the surface structure of the sample. To exemplify this question, Vidal et al. [102] electrochemically showed that the UV/ozone cleaning protocol strongly perturbed the surface structure of different shaped Pt nanoparticles. However, in agreement with Somorjai and co-workers [103-105] for shaped Pt nanoparticles and with Kiwi-Minsker [106] with PVP-stabilized $\mathrm{Pd}$ nanocubes, neither the shape nor the size of nanoparticles was affected by the treatment. This contribution clearly evidenced that the key parameter determining the resulting catalytic or electrocatalytic activity is the surface structure but not the shape of the nanoparticles, Figure 10.
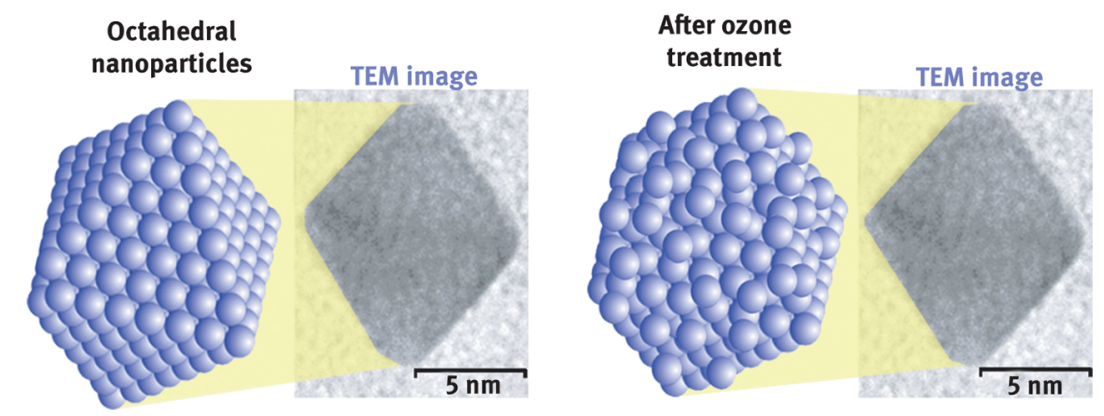

Figure 10: Schematic representation showing two Pt nanoparticles with a similar particle shape (octahedron) but distinct surface structure. Reprinted from Ref. [102], Copyright (2011) with permission from Elsevier.

On the other hand, from the analysis of the oxide formation region, some information about the surface structure can be extracted particularly for Au and Pd surfaces. For Au surfaces, the voltammetric profile of the surface oxide region displayed characteristics features that can be assigned to specific surface structures. This 
analysis is again based on Au single-crystal electrodes [51,52] and it has been already employed for the characterization of the surface structure of different shape-controlled Au nanoparticles [53-55]. For instance, Figure 11 shows the cyclic voltammogram of octahedral, truncated octahedral, and truncated tetrahexahedra (THH) $\mathrm{Au}$ nanocrystals obtained in $0.5 \mathrm{M} \mathrm{H}_{2} \mathrm{SO}_{4}$ solution [53]. From the different voltammetric features appearing in the surface oxide region and based on the previous information gained with Au single-crystal electrodes, the correlations between particle shape and surface structure of the gold nanocrystals can be properly established. Monzó et al. performed a similar analysis of the oxide formation region in alkaline solution [55]. Cubic and octahedral Au nanoparticles displayed distinct voltammetric features in this region which were compared with those previously reported with Au single-crystal electrodes.

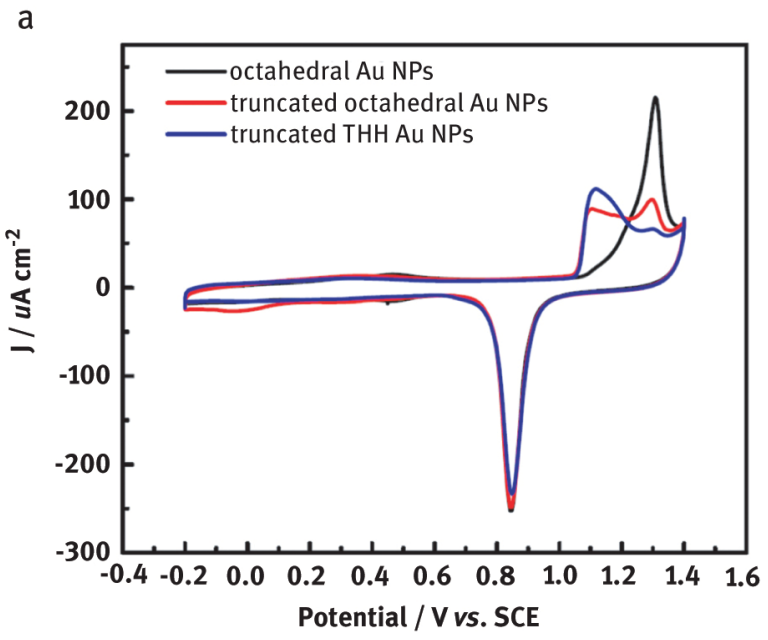

Figure 11: Cyclic voltammogram of octahedral, truncated octahedral and truncated tetrahexahedra (THH) Au nanocrystals obtained in $0.5 \mathrm{M} \mathrm{H}_{2} \mathrm{SO}^{4}$ solution. Sweep rate $50 \mathrm{mV} \mathrm{s}^{-1}$. Reprinted from Ref. [53], Copyright (2014) with permission from Elsevier.

The employ of some UPD processes to characterize the surface structure of different shaped metal nanoparticles is also a very interesting approach because these processes are very sensitive to the surface structure of the electrodes [107, 108]. For instance, Pb UPD has been extensively used on different shaped Au nanoparticles [55, 64, 65, 97, 109-111]. As shown in Figure 12, the Pb UPD displays distinctive features as a function of the surface structure of the shaped Au nanoparticles. The figure clearly shows a preferential $\{100\}$ and $\{111\}$ surface structure for the (C) cubic and (D) octahedral Au samples, respectively. However, for the spherical samples, and independently of their particle size, the Pb UPD profile shows the characteristic response of a polyoriented surface structure.
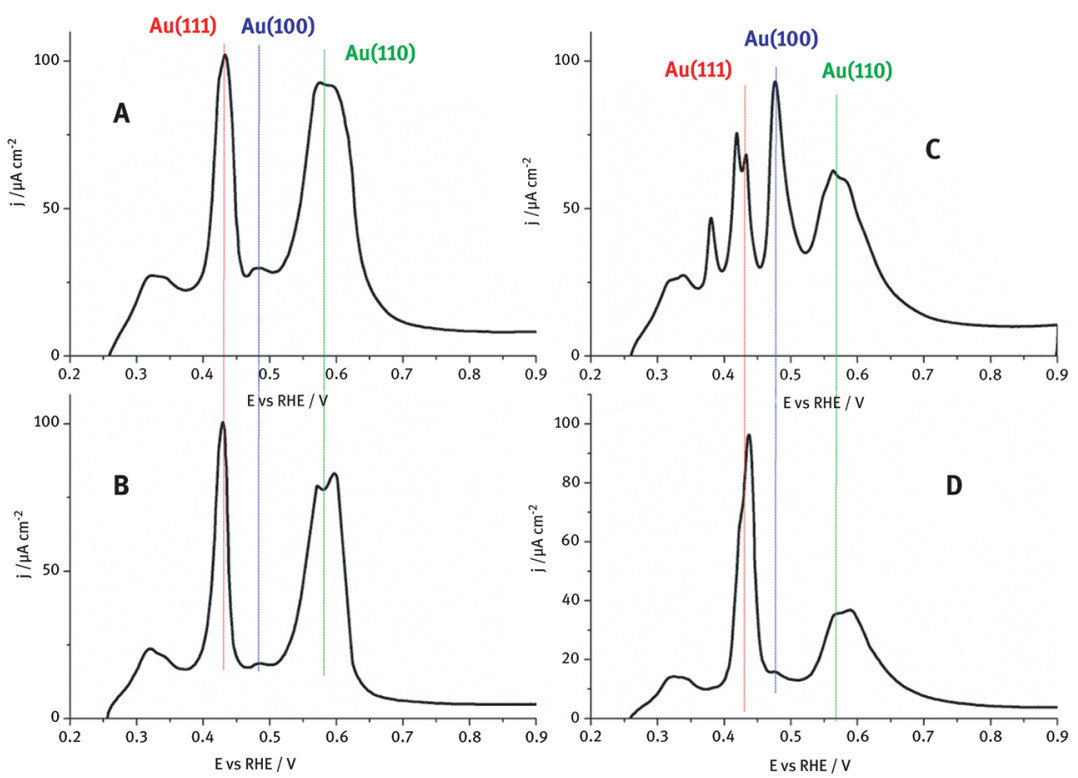

Figure 12: $\mathrm{Pb}$ UPD voltammetric profiles of the unsupported gold nanoparticles in Ar-saturated $0.1 \mathrm{M} \mathrm{NaOH}$ containing $10^{-3} \mathrm{M} \mathrm{Pb}\left(\mathrm{NO}_{3}\right)_{2}:$ (A) spherical $5 \mathrm{~nm}$, (B) spherical $30-40 \mathrm{~nm}$, (C) cubic, and (D) octahedral nanoparticles. Scan rate: 50 $\mathrm{mV} \mathrm{s}^{-1}$. 
Also, $\mathrm{Cu}$ UPD has been used for shaped Pd nanoparticles, particularly for Pd nanocubes [112-114]. In addition, these UPD processes can be also used to calculate the real area of the electrode from the charge involved in the UPD reaction and subsequently normalized with a specific charge density normalization (generally obtained from a polyoriented surface).

Up to now, the different surface structure electrochemical probes discussed are essentially qualitative, that is, from the relative intensity of the characteristic features, it is possible to visualize which surface structure is preferentially present at the surface of the shaped metal nanoparticles. However, it would be much more desirable to have some electrochemical surface probes to quantitatively analyse the surface structure of the nanoparticles. Thus, in the following, we will summarize some of the different approaches proposed for the quantitative characterization of the surface structure of the shaped metal nanoparticles. For Pt surfaces, different alternatives have been reported. Feliu and co-workers proposed a methodology based on the redox behaviour of some adatoms, (Bi, Te, and $\mathrm{Ge})$ spontaneously adsorbed at the surface of different Pt single crystals [99]. They found that $\mathrm{Bi}[115,116]$ and $\mathrm{Te}[117]$ were sensitive to the presence of $\{111\}$ terrace domains, while Ge [118] was sensitive to the $\{100\}$ ordered domains. Interestingly, by using basal planes and stepped surfaces, they observed a linear correlation between the specific response of the adatom and the terrace density of the different Pt electrodes (Figure 13). In this way, for any Pt surface, after performing the Bi and Ge experiments, and using these calibration plots, the quantification of the $\{111\}$ and $\{100\}$ oriented domains present at the surface is possible.
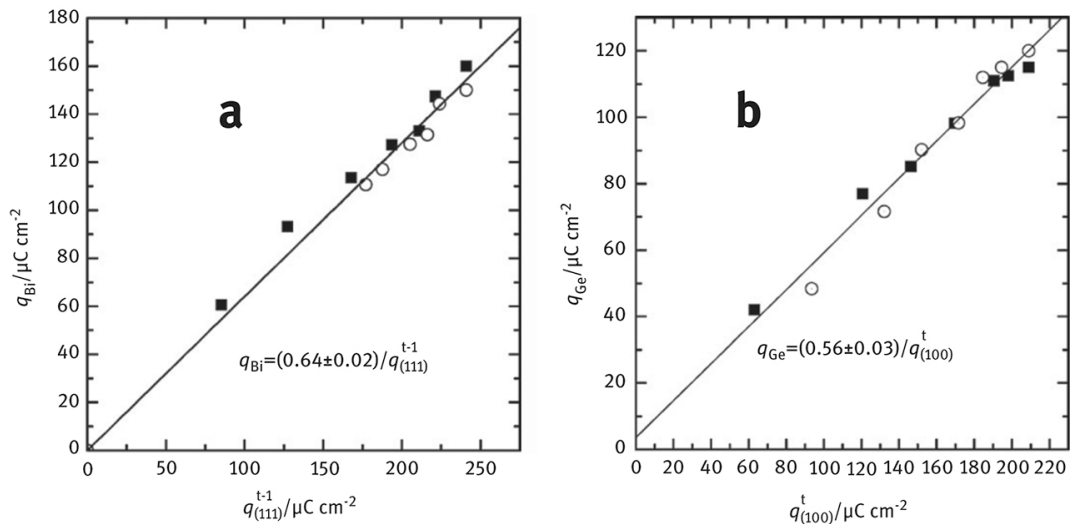

Figure 13: (a) Charge density values of the bismuth redox peak vs. the charge associated with the (111) sites on the terrace. ( $\square) \operatorname{Pt}(n, n, n-2)$ electrodes; (O) $\operatorname{Pt}(n+1, n-1, n-1)$ electrodes. (b) Charge density values of the germanium redox peak vs. the charge associated with the (100) sites on the terrace: $(\boldsymbol{\square}) \operatorname{Pt}(2 n-1,1,1)$ electrodes; (O) $\operatorname{Pt}(n, 1,0)$ electrodes. Reproduced from Ref. [99] with permission from The Royal Society of Chemistry.

This methodology has been satisfactorily applied to quantify the $\{111\}$ and $\{100\}$ oriented domains at the surface of different shape-controlled Pt nanoparticles [99, 116] and it is now worldwide adapted [119-124]. Using a similar concept, other surface structure-sensitive reactions have been proposed including the desorption-adsorption of hydroquinone-derived adlayers (Figure 14) [125] and the reduction of acetaldehyde [126] for $\{111\}$ domains, and the ammonia electrooxidation [127] for $\{100\}$ domains. 


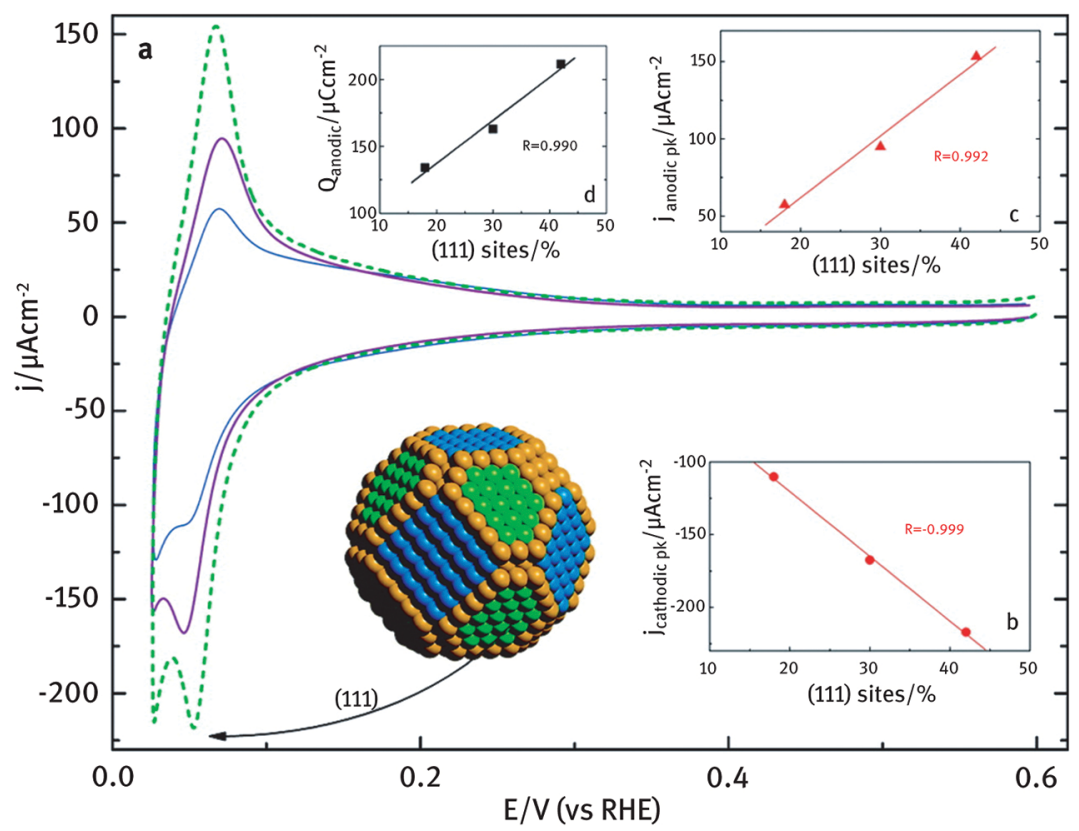

Figure 14: (a) Cyclic voltammograms of different shaped Pt nanoparticle in $2 \mathrm{mM} \mathrm{H}_{2} \mathrm{Q}+0.5 \mathrm{M} \mathrm{H}_{2} \mathrm{SO}_{4}$ solution: (i) thick dashed line (green) for nano-Pt(111)-(110), (ii) medium-thickness solid line (purple) for nano-Pt(111)-(100), and (iii) thin solid line (blue) for nano-Pt(100). Scan rate $50 \mathrm{mV} \mathrm{s}^{-1}$. Insets show (b) the cathodic peak current densities, (c) the anodic peak current densities, and (d) the total anodic charge densities as a function of the density of $\{111\}$ surface sites, expressed as a percent of the electrochemically available surface in each sample. Reprinted with permission from Ref [125]. Copyright (2010) American Chemical Society.

For Au surfaces, El-Deab et al. [128-131] showed that the reductive desorption of different thiol compounds such as cysteine, mercaptoacetic acid, or cystamine can be used to obtain the fraction of the surface sites in various gold nanostructures. In this regard, it is worth noting that although the Pb UPD has been widely used for different shaped $\mathrm{Au}$ nanoparticles, to the best of our knowledge, a quantitative analysis of this Pb UPD voltammetric profile is still missing.

Similarly, but for Pd surfaces, Cu UPD can be used to quantify the $\{100\},\{110\}$, and $\{111\}$ surface domains. To illustrate this analysis, Figure 15 shows the $\mathrm{Cu}$ UPD obtained with Pd nanocubes also including the corresponding fitting using five Lorentzians located at the characteristic contributions of the $\{100\},\{110\}$, and $\{111\}$ surface domains (based on similar experiments on Pd single crystals [132, 133]). The percentage of sites of a given surface is calculated by normalizing the area under its corresponding signal also taking into consideration the calculated charges for a complete Cu monolayer on $\mathrm{Pd}(111), \operatorname{Pd}(100)$, and $\mathrm{Pd}(110)$ single crystals (486, 421, and $297 \mu \mathrm{C} \mathrm{cm}^{-2}$, respectively). In this particular case, the percentage of $\{100\}$ surface sites on the Pd nanocubes was found to be $57 \pm 3 \%$ [113].

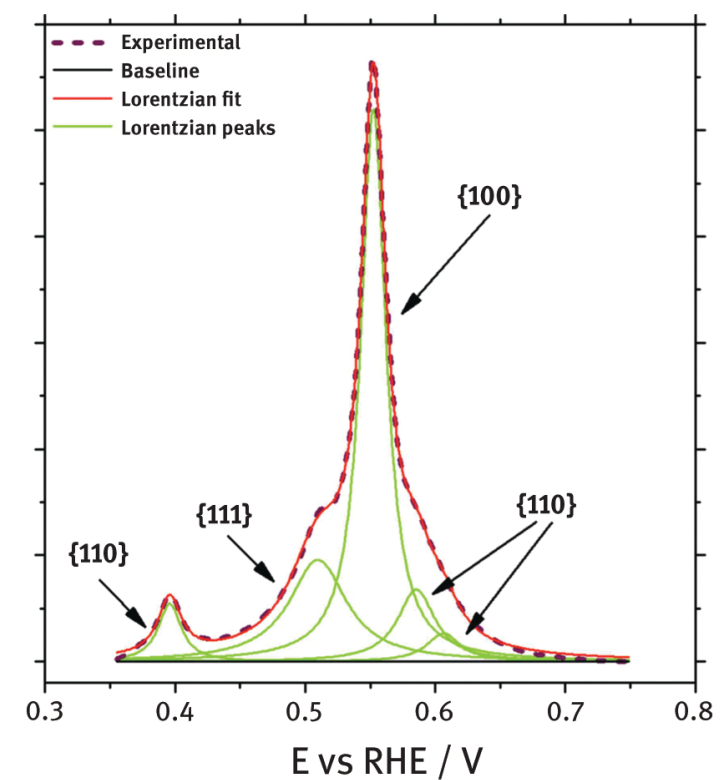


Figure 15: $\mathrm{Cu}$ UPD fitting on the Pd nanocubes. Test solution $0.1 \mathrm{M} \mathrm{H}_{2} \mathrm{SO}_{4}+1 \mathrm{mM} \mathrm{CuSO}_{4}+1 \mathrm{mM} \mathrm{NaCl}$, scan rate $50 \mathrm{mV}$ $\mathrm{s}^{-1}$. Reproduced from [113] with permission of the International Union of Crystallography.

Obviously, it is worth noting that all these previous methodologies are particularly interesting for pure metal nanoparticles ( $\mathrm{Pt}, \mathrm{Pd}$, and $\mathrm{Au}$ ). However, it is also evident that the increasing use of more complex systems including alloys, core-shell, nanoframes, high-index-faceted (convex or concave) and excavated metal nanoparticles, among others, makes this electrochemical surface structure characterization including a correct determination of the electroactive surface area [48-50,134] much more difficult. These are remaining challenges to be overcome in the forthcoming years.

\subsection{Electrocatalysis on shape-controlled metal nanoparticles: representative cases for relevant electrochemical reactions}

In this next section, we will review some of the most relevant advances on the use of shape-controlled metal nanoparticles in electrocatalysis. This section will cover reactions of interest for low-temperature fuel cells such as formic acid, methanol and ethanol electrooxidations and oxygen reduction, and also other relevant electrochemical reactions such as $\mathrm{CO}_{2}$ electroreduction. However, it is worth mentioning that this section will only include contributions that fulfil with the requirement of surface cleanness and that contribute to a better understanding of the correlations between surface structure/shape and electrochemical reactivity.

To the best of our knowledge, the first examples of shape-controlled metal nanoparticles in electrocatalysis were reported by our group in 2004 [71, 72]. In these contributions, we synthetized cubic Pt nanoparticles using a similar methodology to that described in 1996 by El-Sayed and co-workers [14] and based in a chemical reduction of $\mathrm{K}_{2} \mathrm{PtCl}_{4}$ by $\mathrm{H}_{2}$ in the presence of NaPA. As expected from Figure 1, the cubic Pt nanoparticles displayed a preferential $\{100\}$ surface structure as deduced from the voltammetric profile of the nanoparticles obtained in $0.5 \mathrm{M} \mathrm{H}_{2} \mathrm{SO}_{4}$. The cubic Pt nanoparticles were tested towards ammonia electrooxidation in alkaline solution and showed an enhanced activity due to extreme sensitivity of this reaction to the presence of $\{100\}$ sites as illustrated from Pt single-crystal studies $[135,136]$. These results clearly evidenced that by tuning the surface structure of the nanoparticles by means of controlling their particle shape, improved electrocatalytic activity were achieved. From these first examples, many other contributions have been and are being currently published reporting a clear correlation between particle shape and or surface structure and enhanced electrocatalytic activities. In fact, there already exist several and excellent reviews related to the application of shaped metal nanoparticles for different electrocatalytic reactions [6, 7, 20, 137-150].

\section{Formic acid electrooxidation}

Formic acid electrooxidation is a very valuable reaction, not only because it is a model reaction for a twoelectron-transfer reaction, but also for its possible use as fuel in the so-called direct formic acid fuel cells (DFAFCs) [5, 151-153]. On metal surfaces, this process is accepted to proceed through a dual path mechanism [154-156]: a direct pathway via an adsorbed active intermediate (the nature and role of this active intermediate are still under strong discussion at fundamental level) that readily yield to $\mathrm{CO}_{2}$, and a second pathway involving the formation of adsorbed $\mathrm{CO}$, which is considered to be a poisoning intermediate, that is subsequently oxidized to $\mathrm{CO}_{2}$ at high overpotentials. On Pt surfaces, both reaction paths are structure sensitive, and the $\mathrm{Pt}(100)$ surface is the most active but also the most sensitive to the CO poisoning [152]. This surface structure sensitivity was first observed with shape-controlled Pt nanoparticles by Tian and co-workers [29] who reported that tetrahexahedral (THH) Pt nanocrystals displayed, in comparison with polycrystalline Pt nanospheres and commercial Pt/C catalyst (E-TEK Co., Ltd), a clear enhanced activity (about 4 and 3 times higher than for the nanospheres and the commercial catalyst, respectively).

The effect of the presence of $\{100\} \mathrm{Pt}$ domains at the surface of the nanoparticles was subsequently explored by our group by using cubic Pt nanoparticles [78, 157]. In brief, spherical, cubic, cubo-octahedral, and tetrahedral-octahedral Pt nanoparticles were prepared in the presence of NaPA and employed towards formic acid electrooxidation. As expected, we observed clear similarities between the response of the nanoparticles and those obtained with the model Pt surfaces. In particular, the cubic Pt nanoparticles, that is, those nanoparticles having a preferential (100) orientation showed the highest activity as well as the fastest CO poisoning in good agreement with previous single-crystal experiments [152].

Since these initial works, some other contributions have reported enhanced activities towards formic acid electrooxidation by using shaped Pt nanoparticles including concave Pt nanocrystals having $\{411\}$ high-index facets [158], trapezohedral (TPH) Pt nanocrystals enclosed by \{522\} high-index facets [159], highly concave Pt 
nanoframes [160], truncated octahedral, cuboctahedral, and cubic shape-controlled ( $<10 \mathrm{~nm}) \mathrm{Pt}$ nanoparticles [34], and concave Pt nanocubes [161], among others.

Shape-controlled Pd nanoparticles have been also extensively used for formic acid electrooxidation due to their unique properties. On Pd surfaces, the dehydration step is strongly hindered (CO is not spontaneously formed) and, therefore, the reaction directly proceeds through the direct path. In addition, the onset oxidation potential is about $200 \mathrm{mV}$ lower than that observed for Pt [153, 162]. Also, the reaction is structure sensitive and the $\operatorname{Pd}(100)$ was found to be the most active surface among the basal planes, both in sulphuric and in perchloric acid solutions [163, 164]. In this regard, Jin et al. [112] and Zhang et al. [165] reported the first examples of shaped Pd nanoparticles towards formic acid electrooxidation. Jin et al. evaluated a collection of shaped Pd nanoparticles including cubes, truncated cubes, cuboctahedra, truncated octahedra, and octahedral [112]. As expected from single crystals, the activity was found to be dependent on the fraction of $\{100\}$ domains, and the cubic nanoparticles displayed the highest activity, Figure 16. Similarly, Zhang et al. employed rhombic dodecahedra and cubic Pd nanoparticles and again the cubic ones displayed the highest electrocatalytic activity towards formic acid electrooxidation [165].

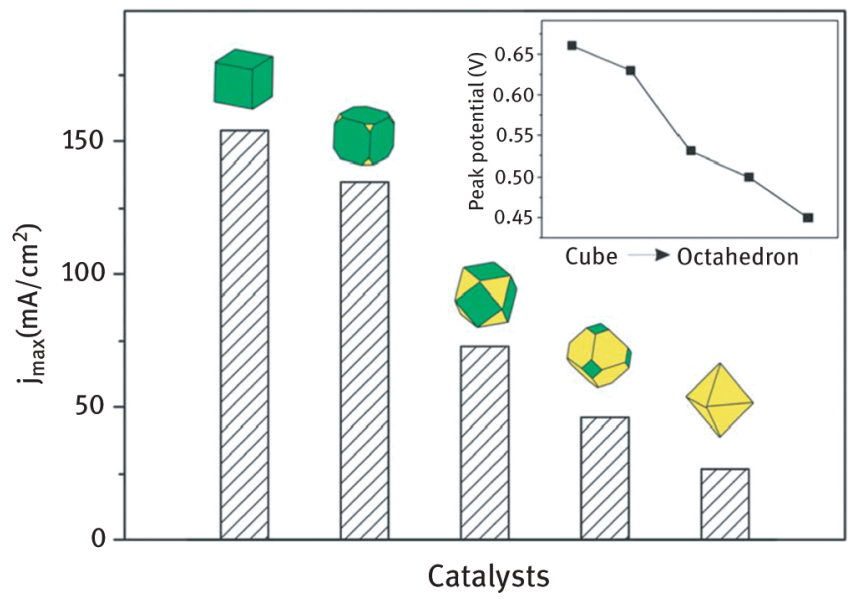

Figure 16: Maximum current densities for formic acid electrooxidation (normalized to the electrochemical surface area) for different shaped $\mathrm{Pd}$ nanoparticles in $0.1 \mathrm{M} \mathrm{HClO}_{4}+2 \mathrm{M} \mathrm{HCOOH}$ at $10 \mathrm{mV} \mathrm{s}^{-1}$. The inset shows their corresponding peak potentials. Reproduced from Ref. [112]with permission from The Royal Society of Chemistry.

Different contributions were subsequently reported using very different shaped Pd samples including, among others, cubic and spherical Pd nanoparticles [75]; different concave Pd nanocrystals (nanocubes, fivefold twinned nanorods and right bipyramids) [166]; twinned icosahedra, cubic and octahedral Pd nanoparticles [167]; cubic, octahedra, icosahedra, and right bipyramids [168-170]; monodispersed (50 nm) palladium nanocrystals, including cubes, octahedral, and rhombic dodecahedra [171]; length tuneable penta-twinned Pd nanorods [172]; tetrahedral Pd nanocrystals [173]; cubes [174]; penta-twinned Pd nanowires [175]; and branched Pd nanoparticles [176].

The incorporation of a second element both on $\mathrm{Pt}$ and on $\mathrm{Pd}$ surfaces is a classical approach to enhance their electrocatalytic properties towards formic acid electrooxidation $[153,177,178]$. The resulting enhancements can be justified by third-body, electronic, and/or bifunctional effects. Third-body effects take place when the second element acts as a mere spectator, blocking certain surface sites, thus effectively inhibiting some undesired reactions. On the other hand, electronic effects happen when the second element changes the electronic properties of the bare surface. In this aspect, the so-called d-band centre model [179] is widely accepted and explains the possible modifications of the electronic properties and binding energies of a transition metal surface, after the incorporation of a second metal, and their correlations with the resulting catalytic activity. Finally, bifunctional effects take place if the second metal facilitates the reaction mechanism by providing some functional groups at low potentials. Obviously, this strategy has been also employed with shape-controlled metal nanoparticles by exploring two different approaches: (a) shape-controlled Pt or Pd alloy-based nanoparticles and (b) decoration of the surface of the shaped Pt and Pd nanoparticles with different adatoms.

Several and outstanding contributions have been reported on the use of shape-controlled $\mathrm{Pt}$ and $\mathrm{Pd}$ alloy nanoparticles towards formic acid electrooxidation [21, 139, 143, 144, 180]. These contributions include PdPt alloy nanocubes with tunable compositions [181], $\mathrm{Pt}_{3}$ Fe nanocubes [182], PtCu nanocubes of different atomic composition $\left(\mathrm{Pt}_{\mathrm{x}} \mathrm{Cu}_{100-\mathrm{x}}(x=54-80\right.$ atomic \%) [183, 184], Pt-Cu nanooctahedra [185, 186], PtMn nanocubes [187], different polyhedral AuPd core-shell structures $(60-80 \mathrm{~nm}$ ) having high-index facets (concave trisoctahedral (TOH) and hexoctahedral (HOH) crystals both with $\{\mathrm{hkl}\}$ facets and THH crystals with $\{\mathrm{hk0}\}$ facets)) [188], HOH AuPd alloy nanoparticles [189], THH PdPt alloy nanocrystals [190], excavated rhombic dodecahedral (ERD) 
$\mathrm{PtCu}_{3}$ alloy nanoparticles[191], cubic Pd-Ni-Pt core-sandwich-shell nanoparticles [192], and PdCu bimetallic tripods [193], among many others. Obviously, the optimal atomic composition and particle shape depends on each specific system.

On the other hand, the use of certain adatoms to selectively decorate the surface of different shape-controlled metal nanoparticles towards formic acid electrooxidation has been also explored in different contributions. Feliu and co-workers employed different adatoms including Bi [194, 195], Pd [196, 197], Sb [198], and more recently $\mathrm{Tl}$ [199] and $\mathrm{Pb}$ [200] as surface modifiers on different shaped Pt nanoparticles. Sun and co-workers also used $\mathrm{Bi}$ [194] and Au [201] with THH Pt nanocrystals. Improved electrocatalytic activities were obtained in all these cases, although the enhancement factor is determined by the surface structure of the substrate and the nature and coverage of each adatom.

Using a similar concept, Yang and Lee reported a very interesting approach by which the surface of gold octahedral nanocrystals was decorated with the epitaxially deposited Pt atoms [54]. In this case, the shaped gold nanoparticles acted as substrate to control the Pt deposition which varied from fully covered multiple overlayers (about 5 monolayers) to atomically dispersed sub-monolayer ( 0.05 monolayer). The electrocatalytic activity of the Pt modified gold nanoparticles was found to be strongly dependent of the Pt coverage and, for a Pt coverage of about 0.05 monolayer, a huge enhancement was observed.

\section{Methanol electrooxidation}

Methanol electrooxidation is also of great interest for fuel cell applications [5, 202]. On metal surfaces, this reaction is also known to be sensitive to the surface structure [203-205] and therefore, many different shaped controlled metal nanoparticles have been evaluated. For Pt nanoparticles, we clearly showed the effect of the shape/surface structure of the nanoparticles by using cubic, octahedral/tetrahedral, and truncated octahedral Pt nanoparticles (about 8-10 nm) prepared with NaPA [78]. The results obtained indicated that the octahedral $\mathrm{Pt}$ nanoparticles displayed the highest activity towards methanol electrooxidation in $0.5 \mathrm{M} \mathrm{H}_{2} \mathrm{SO}_{4}$ solution.

Chen et al. studied very small (about $3.5 \mathrm{~nm}$ ) cubic Pt nanoparticles prepared in the absence of surfactant [206]. The activity of the cubic nanoparticles towards methanol oxidation in $\mathrm{KOH}$ solution was found to be significantly higher than that obtained with a commercial $\mathrm{Pt} / \mathrm{C}$ catalyst.

Interesting contributions have been reported for $\mathrm{Pd}$ nanoparticles due to their intrinsic $\mathrm{CO}$ tolerance. Arjona et al. prepared $10 \mathrm{~nm}$ cubic Pd nanoparticles and reported an enhanced activity towards methanol oxidation in alkaline solution in comparison with a commercial Pd catalyst [207]. Cubic Pd nanoparticles were also evaluated toward methanol electrooxidation by Kannan et al. who reported a clear enhancement (about 11 times) in comparison with a commercial Pd/C catalyst in $\mathrm{HClO}_{4}$ solution [208]. Qin et al. employed star-like and concave Pd nanoparticles which also showed an enhanced activity (more than three times) in comparison with commercial Pd/C in alkaline medium [209].

As previously discussed for formic acid electrooxidation, the use of shaped controlled $\mathrm{Pt}$ and $\mathrm{Pd}$ alloy nanoparticles and the adatom surface decoration of different shaped metal nanoparticles are widely accepted strategies for obtaining better electrocatalysts for methanol electrooxidation. Two contributions will be briefly discussed to exemplify these two different approaches, both dealing with the Pt-Ru system which has been widely considered for this reaction and where the incorporation of Ru promotes the formation of oxygen species at low potential, thus facilitating the reaction through a bifunctional mechanism [210,211] On the one hand, Sun and co-workers reported that Ru-decorated THH Pt nanoparticles displayed a high tolerance to CO poisoning and an onset oxidation potential shift of about $100 \mathrm{mV}$ towards more negative potentials [212]. In comparison with Ru-decorated Pt/C and with commercial PtRu alloy nanoparticle catalyst, these Ru-decorated THH Pt NPs showed a higher activity in the low potential range.

On the other hand, very recently, Huang et al. reported the preparation of shaped controlled PtRu alloy nanoparticles and their use for methanol electrooxidation in $\mathrm{HClO}_{4}$ solution [213]. In more detail, $\mathrm{PtRu}$ nanowires, nanocubes, and nanorods were synthetized through a one-step solvothermal method. The results indicated that the $\{111\}$-terminated PtRu nanowires displayed higher activity and stability than those obtained with the $\{100\}$-terminate PtRu nanocubes.

Obviously, many other systems have been evaluated for methanol electrooxidation including PtPd nanocubes [214]; PtPd nanotetrahedra [215]; octahedral PtPd alloy nanoparticles [216]; multiply twinned PtPd nanoicosahedra [217]; concave PtPd nanocubes [218]; cubic, rod-like, quasi spherical, concave cubic, and TOH $\mathrm{Au}$ nanoparticles [219]; plate-like and truncated octahedral $\mathrm{Cu}$ nanocrystals [220]; concave PtCu [221] and PtPdCu [222] nanocubes; Pt3Co nanoflowers and nanocubes [223]; PtZn nanocubes [224]; platinum-copper nanowires [225]; deeply excavated Pt3Co nanocubes [226]; excavated octahedral Pt-Co alloy nanocrystals [227]; 
platinum-copper rhombic dodecahedral (RD) nanoframes [228]; platinum-silver alloyed octahedral nanocrystals [229]; screw-like PdPt nanowires [230]; and Cu-rich PtCu octahedral alloy nanocrystals [231], among others.

\section{Ethanol electrooxidation}

Ethanol electrooxidation has been also the subject of innumerable studies. Due to difficulties in C-C bond cleavage, the ethanol oxidation, however, is often incomplete, resulting in a number of by-products other than $\mathrm{CO}_{2}$ such as acetaldehyde and acetic acid. As previously stated for methanol, on metal surfaces, this reaction is also surface structure sensitive [232-234] and, consequently, susceptible of being studied on shaped controlled metal nanoparticles. Tian and co-workers [29] reported that THH Pt nanocrystals displayed an enhanced activity towards ethanol electrooxidation in comparison with polycrystalline Pt nanospheres and commercial $\mathrm{Pt} / \mathrm{C}$ catalyst (E-TEK Co.). Wei et al. also reported that concave THH Pt nanocrystals (bounded by $\{910\}$ faces) prepared in deep eutectic solvents were more active (about 2 times) than a Pt black catalyst in $\mathrm{HClO}_{4}$ solution [235]. Also, Huang et al. showed that concave polyhedral Pt nanoparticles having $\{411\}$ high-index faces exhibited an enhanced electrocatalytic activity over commercial $\mathrm{Pt}$ catalysts towards ethanol oxidation reaction in $\mathrm{HClO}_{4}$ [158]. Similarly, Zhang et al. also reported a higher ethanol oxidation activity (in $\mathrm{HClO}_{4}$ ) with multipod and concave Pt nanocrystals, mainly exposing $\{211\}$ and $\{411\}$ high-index facets, respectively, than that observed with Pt/C and Pt nanocubes [236]. Interestingly, Zhou et al. showed that THH Pt nanoparticles, prepared from an electrochemical perturbation of Pt nanocubes $(10 \mathrm{~nm})$ in $\mathrm{H}_{2} \mathrm{SO}_{4}$, displayed a remarkable increase in activity towards ethanol oxidation, in comparison with the original $\mathrm{Pt}$ nanocubes and commercial $\mathrm{Pt}$ nanoparticles [237]. All these previous contributions clearly point out the benefits of having nanoparticles containing high-index surfaces for ethanol electrooxidation. However, "low-index" metal nanoparticles have been also evaluated and, for instance, Busó-Rogero et al. systematically evaluated the ethanol oxidation on different shaped Pt nanoparticles including spherical, cubic, and octahedral Pt nanoparticles both in acidic and in alkaline solution [238-240]. The results obtained clearly agreed with those previously found with Pt single-crystal electrodes [232-234].

Apart from $\mathrm{Pt}$, shaped controlled $\mathrm{Pd}$ and $\mathrm{Rh}$ nanoparticles have also been employed for ethanol electrooxidation including cubic Pd nanoparticles [207], concave Pd nanocubes [241], and THH Rh nanocrystals [242]. Obviously, several shaped metal alloy nanoparticles have been considered for this reaction. Among others, Chen et al. used different shaped PtPd alloys supported in graphene for ethanol electrooxidation [243]. Higher electrocatalytic activities and better tolerance to poisoning were observed for all shapes in comparison with Pt nanoflowers, Pd nanoparticles, and unshaped PtPd nanoparticles supported on carbon black. Rao et al. employed different PtRh alloy nanocubes supported on graphene [244]. In this case, a Pt:Rh atomic ratio = 9:1 was found to be the most convenient for ethanol oxidation. High-index PtRh nanocrystals including \{830\}-bounded THH and \{311\}-bounded trapezohedron (TPH) have been also tested [30]. Hong et al. evaluated AuPd octapodal nanoparticles in comparison with flower-like AuPd alloy nanoparticles and a commercial $\mathrm{Pd} / \mathrm{C}$ catalyst [245]. Also, Zhang et al. reported the preparation of monodisperse $\mathrm{Au}-\mathrm{Pd}$ alloy nanoparticles with systematic shape evolution from $\mathrm{RD}$ to $\mathrm{TOH}$, and $\mathrm{HOH}$ structures by varying the concentration of surfactant in the surfactant-mediated synthesis [246]. The catalytic activities toward ethanol electrooxidation were in the order of $\mathrm{HOH}>\mathrm{RD}>\mathrm{TOH}$, following the same order of their corresponding surface energies. More recently, Rizo et al. studied the ethanol electrooxidation on PtSn nanocubes [247]. They observed that the electrochemical activity of the cubic Pt-Sn nanoparticles was found to be about three times higher than that obtained with unshaped $\mathrm{Pt}-\mathrm{Sn}$ nanoparticles and six times higher than that of Pt nanocubes. Very interestingly, Erini et al. have recently explored the use of octahedral PtNiRh nanoparticles, having a fixed Pt:Ni ratio and different Rh contents (between 1 and 6 atomic \%), towards ethanol electrooxidation [248]. These multimetallic samples displayed a good electrochemical activity and stability towards ethanol oxidation in alkaline solution.

On the other hand, ethanol electrooxidation is also suitable of being optimized by using adatom-decorated shaped metal nanoparticles. For instance, Wang et al. studied Bi-decorated THH Pd nanoparticles towards ethanol oxidation in alkaline medium [249]. The activity of the Bi-decorated samples was found to be about 3 and 12 times higher than that of bare THH Pd nanoparticles and a Pd/C catalyst, respectively, at the optimum Bi coverage. Similarly, Busó-Rogero et al. employed different adatoms ( $\mathrm{Sn}, \mathrm{Rh}, \mathrm{Ru}$, and $\mathrm{Pb}$ ) to systematically decorate the surface of different shaped Pt nanoparticles and studied their electrocatalytic properties for ethanol electrooxidation both in acidic and in alkaline solutions [250]. However, in all cases, the enhancement was very limited. 


\section{Oxygen reduction reaction}

Oxygen reduction reaction (ORR) is, without any doubt, one of the most studied reactions in electrocatalysis $[139,143,251-255]$. Among pure metals, Pt presents the highest electrocatalytic activity for the $4 \mathrm{e}^{-}$pathway to water [256-259]. ORR is also known to be sensitive not only to the surface structure of the electrode but also to the used electrolyte [260]. This surface structure sensitivity has been also demonstrated on different shaped metal nanoparticles. Thus, for instance, Inaba et al. used cubic Pt nanoparticles prepared in the presence of $\mathrm{NaPA}$ which displayed a high activity for ORR in $\mathrm{H}_{2} \mathrm{SO}_{4}$ solution [261]. Similarly, Sánchez-Sánchez et al. also evaluated the ORR activity of different shape-controlled Pt nanoparticles by using the scanning electrochemical microscope (SECM) [262]. The results pointed out the good agreement between the ORR activity of the shaped $\mathrm{Pt}$ nanoparticles and the results obtained with Pt single-crystal electrodes. In this regard, Tripkovic et al. [263] reported a density functional theory (DFT) study dealing with the effect of the shape of Pt nanoparticles on the ORR activity in a non-adsorbing electrolyte. The results showed that the tetrahedral Pt nanoparticles provided the highest activities and the cubic Pt nanoparticles the least actives. These findings are in good agreement with previous experimental observations on shape-controlled Pt nanoparticles [262] in a non-adsorbing electrolyte such as $\mathrm{HClO}_{4}$. In addition, taking into account that stepped Pt single-crystal surfaces usually displayed higher ORR catalytic activity than that observed for the basal surfaces [256-259], several high-index Pt nanoparticles have been explored towards ORR. Readers interested in this topic are referred to some relevant reviews [141, 147-149].

Shaped Pd nanoparticles have been also extensively tested for ORR. Xiao et al. used Pd nanorods and observed a specific activity about 10 times higher than that of Pd spherical nanoparticles [264]. Shao et al. [265], Erikson et al. [73, 74], and Lee et al. [266] also reported enhanced ORR activities by using Pd nanocubes containing a preferential $\{100\}$ surface structure. Relevant reviews about the electrocatalysis of Pd-based nanomaterials for ORR are already available in the literature $[267,268]$.

Among the vast literature existing dealing with the use of shaped metal alloy nanoparticles towards ORR $[32,33,37,139,143,146,150,180,253,255,262,269-272]$, the system Pt-Ni is particularly relevant. Stamenkovic et al. published that the ORR-specific activity at $0.9 \mathrm{~V}$ of a single-crystal $\mathrm{Pt}_{3} \mathrm{Ni}(111)$ surface was about 90 times higher than that of a commercial $\mathrm{Pt} / \mathrm{C}$ in $0.1 \mathrm{M} \mathrm{HClO}_{4}$ solution [273]. This finding clearly marked the efforts towards the preparation of octahedral $\mathrm{Pt}_{3} \mathrm{Ni}$ nanoparticles and their use for ORR [274]. To the best of our knowledge, the first example of shape-controlled $\mathrm{Pt}_{3} \mathrm{Ni}$ nanoparticles was reported by Zhang et al. [275] who prepared $\mathrm{Pt}_{3} \mathrm{Ni}$ octahedra and nanocubes and observed that the ORR-specific activity of the $\mathrm{Pt}_{3} \mathrm{Ni}$ octahedra was about five times higher than that obtained with $\mathrm{Pt}_{3} \mathrm{Ni}$ nanocubes, Figure 17. In addition, the specific and mass activities of $\mathrm{Pt}_{3} \mathrm{Ni}$-octahedra/C were about seven and four times higher than that of a commercial $\mathrm{Pt} / \mathrm{C}$ electrocatalysts.
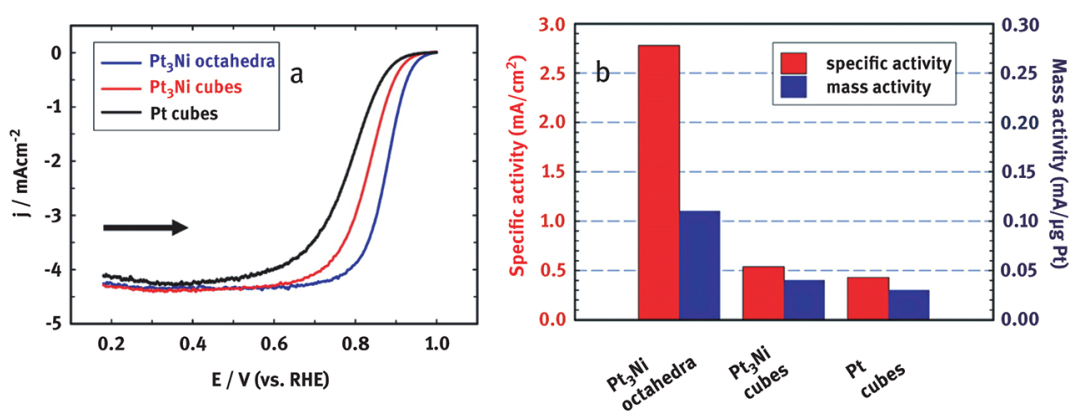

Figure 17: (a) ORR behaviour of $\mathrm{Pt}_{3} \mathrm{Ni}$ nanoctahedra, $\mathrm{Pt}_{3} \mathrm{Ni}$ nanocubes, and $\mathrm{Pt}$ nanocubes supported on a rotating GC disk electrode in $\mathrm{O}_{2}$ saturated $0.1 \mathrm{M} \mathrm{HClO}_{4}$ solution at $20 \mathrm{mV} \mathrm{s}^{-1}$ and at $295 \mathrm{~K}$; rotation rate, $900 \mathrm{rpm}$. (b) Comparison of the ORR activities at $0.9 \mathrm{~V}$ vs. RHE at $295 \mathrm{~K}$. Reprinted with permission from Ref [275]. Copyright (2010) American Chemical Society.

Since this first contribution, many other approaches have been reported and for the readers' interests in this particular topic, we refer to some recent reviews by Strasser et al. in which the most relevant advances are visually summarized (Figure 18) in terms of ORR Pt mass activities [150, 276, 277]. 


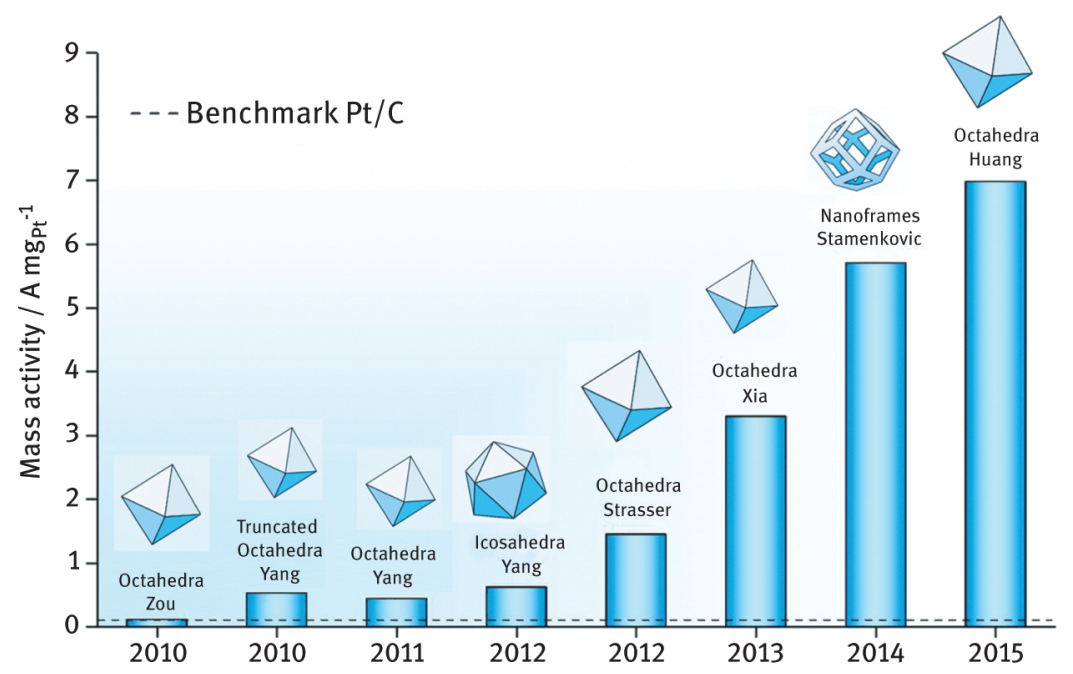

Figure 18: Schematic representation of the performance of the most relevant shaped PtNi nanoparticles (given in A $\mathrm{mg}^{-1}(\mathrm{Pt})$ at $+0.9 \mathrm{~V}$ vsRHE (iR-corrected electrode potential)) towards ORR. Reproduced from Ref. [150] with permission from The Royal Society of Chemistry.

\section{$8 \mathrm{CO}_{2}$ electroreduction}

The electrochemical reduction of $\mathrm{CO}_{2}$ is being the subject of numerous efforts basically with two main objectives: (i) decreasing the $\mathrm{CO}_{2}$ concentration at the atmosphere (this high concentration strongly contributes to the greenhouse effect and climate change) and (ii) transforming this $\mathrm{CO}_{2}$ into valuable chemicals and/or fuels. However, this reaction presents a very complicated chemical reaction mechanism, and multiple electrontransfer processes with manifold coupled consecutives intermediates can take place [278-282]. In addition, and due to its particular structure, $\mathrm{CO}_{2}$ is quite thermodynamically and kinetically stable, requiring usually high overpotentials in order to produce $\mathrm{C} 1, \mathrm{C} 2$, and even $\mathrm{C} 3$ compounds. Also, the hydrogen evolution reaction (HER) is its serious competitive reaction in aqueous solution which give rises to low faradaic efficiencies. Nevertheless, on the other hand, it is also well-established that the product selectivity during the $\mathrm{CO}_{2}$ electroreduction is strongly determined by the nature of the metal and also by the surface structure [283-288].

Therefore, and due to this surface structure sensitivity, different shape-controlled metal nanoparticles have been evaluated. Among others, $\mathrm{Cu}$ is particularly the most interesting metal due its unique properties to convert $\mathrm{CO}_{2}$ into different hydrocarbons, mainly methane $\left(\mathrm{CH}_{4}\right)$ and ethylene $\left(\mathrm{C}_{2} \mathrm{H}_{4}\right)$ [150, 289-292]. For instance, Chen et al. [293] showed that on Cu mesocrystals having $\{100\}$ oriented facets, the faradaic efficiency towards ethylene formation was about 18 times larger than that of methane. Similarly, Nilsson and co-workers used Cu nanocubes to selectively produce ethylene over methane due to their preferential $\{100\}$ surface structure [294, 295]. Also, Kwon et al. [296] reported a selective production of ethylene on $\mathrm{Cu}$ nanocubes prepared using a halide based electrochemical treatment. On these nanocubes, the faradaic efficiency towards ethylene was found to be 1.5 higher than that for polycrystalline copper (at $-1.0 \mathrm{~V}$ vs. RHE). Interestingly, the faradaic efficiency for ethanol was also remarkably high (about $8 \%$ ). Gao et al. tested the behaviour of $\mathrm{Cu}$ nanocubes with tunable $\{100\}$ facet and oxygen/chlorine ion content by using a low-pressure plasma pre-treatment towards $\mathrm{CO}_{2}$ electroreduction [297]. The results obtained displayed high selectivity for ethylene, ethanol, and $n$-propanol and a maximum faradaic efficiency of about $73 \%$ for $\mathrm{C}_{2}$ and $\mathrm{C}_{3}$ compounds. Additionally, $\mathrm{Cu}$ nanocubes with different edge length (24, 44, and $63 \mathrm{~nm}$ ) were also evaluated by Loiudice et al. [298]. Among the samples, the $44 \mathrm{~nm}$ cubic samples displayed the highest faradaic efficiency for ethylene (about $41 \%$ ). More recently, Jeon et al. reported that prism-shaped $\mathrm{Cu}$ catalysts displayed a high $\mathrm{C}_{2} \mathrm{H}_{4}$ production (about four times higher) in comparison with that observed with a planar $\mathrm{Cu}$ electrode (figure 19) [299].

Other shapes such as $\mathrm{Cu}$ nanowires [300-302], hierarchical $\mathrm{Cu}$ pillar [303], and $\mathrm{Cu}$ nanofoam [304] have been also explored.

Different shaped silver (Ag) nanoparticles were also evaluated for $\mathrm{CO}_{2}$ electroreduction. For instance, Liu et al. showed that triangular Ag nanoplates having $\{100\}$ domains exhibited improved electrochemical properties towards CO in comparison with similarly sized Ag nanoparticles (SS-Ag-NPs) and bulk Ag [305].

Lately, Peng et al. employed a simple anodization treatment to prepare preferentially oriented metallic Ag nanoparticles with high activity and high selectivity for the reduction of $\mathrm{CO}_{2}$ to $\mathrm{CO}$ [306]. The $\{110\}$ and $\{100\}$ preferentially oriented $\mathrm{Ag}$ nanocrystals displayed faradaic efficiency of about $97 \%$ (at $-0.69 \mathrm{~V}$ vs. RHE), which 
was significantly higher than that obtained with polycrystalline $\mathrm{Ag}$ ( $60 \%$ at $-0.87 \mathrm{~V}$ vs. RHE). Other systems such as Ag nanocorals [307] and Ag nanoporous electrodes [308] have also exhibited high selectivity towards CO.

Shape-controlled Pd nanoparticles are particularly interesting to produce formate. Klinkova et al. [309] employed different shaped $\mathrm{Pd}$ nanoparticles for the electroreduction of $\mathrm{CO}_{2}$ to formate. The results obtained suggested that the presence of high-index surfaces enhances the electrocatalytic performance (Figure 20). Thus, the high-index samples displayed a faradaic efficiency of about $97 \%$ towards formate at a low overpotential $(-0.2$ V vs. RHE).

Interestingly, Gao et al. have recently published a perspective summarizing the behaviour of different Pdand Pd-based nanostructures towards $\mathrm{CO}_{2}$ electroreduction mainly towards formate [310]. Moreover, the electrochemical reduction of $\mathrm{CO}_{2}$ towards $\mathrm{CO}$ is also possible on Pd surfaces. In this regard, Huang et al. reported the $\mathrm{CO}$ production on octahedral and icosahedral Pd nanoparticles. The results obtained showed that the faradaic efficiency for CO production on Pd icosahedra (about $91 \%$ at $0.8 \mathrm{~V}$ vs. RHE) was found to be almost two times higher than that observed on Pd octahedra [311].

Some zinc ( $\mathrm{Zn})$-shaped nanostructures have been also studied. In particular, nanodendrites [312] and hexagonal [313] Zn electrodes have demonstrated remarkable activities for the selective production of CO.

Besides, different Bi nanostructures have been also considered for the selective production of formate. Thus, Bi nanodendrites $[314,315]$ and Bi nanoflakes [316] have displayed very high faradic efficiencies towards formate $(96.4 \%$ and about $100 \%$ respectively). On the other hand, in acetonitrile solution, shaped Bi nanoparticles have displayed very high faradaic efficiency (about 96.1\%) towards CO production [317].

Furthermore, some shaped Au nanoparticles have been also explored including nanowires [318, 319], concave RD [320], pore-like or pillar-like structures [321], and nanocubes [322], among others. In all these cases, a selective conversion of $\mathrm{CO}_{2}$ to $\mathrm{CO}$ is observed.

To complete this section, it is worth noting some relevant contributions dealing with the use of metal alloy, core-shell, and adatom-decorated shaped electrocatalysts for $\mathrm{CO}_{2}$ electroreduction. Very briefly, Zhao et al. employed $\mathrm{Cu}-\mathrm{Pt}$ alloy nanocube [323] with different $\mathrm{Pt}: \mathrm{Cu}$ atomic compositions. The reduction potential becomes more positive with increasing $\mathrm{Cu}$ content, and $\mathrm{Cu}_{85} \mathrm{Pt}_{15}$ nanocubes were found to be the most convenient system for $\mathrm{CO}_{2}$ reduction.

On the other hand, Monzó et al. [322] tested $\mathrm{Au}-\mathrm{Cu}$ core-shell nanoparticles in which Au nanocubes were systematically covered with increasing $\mathrm{Cu}$ layers. As illustrated in Figure 21, the product selectivity for the $\mathrm{CO}_{2}$ reduction was clearly dependent on the thickness of the $\mathrm{Cu}$ shells.

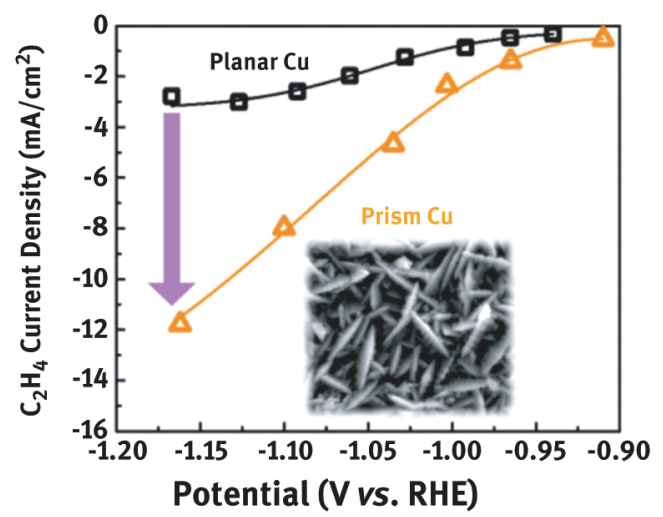

Figure 19: Partial current densities vs. applied potential for $\mathrm{C}_{2} \mathrm{H}_{4}$ of a planar $\mathrm{Cu}$ foil and a Prism Cu electrode. Reprinted with permission from Ref [299]. Copyright (2018) American Chemical Society. 


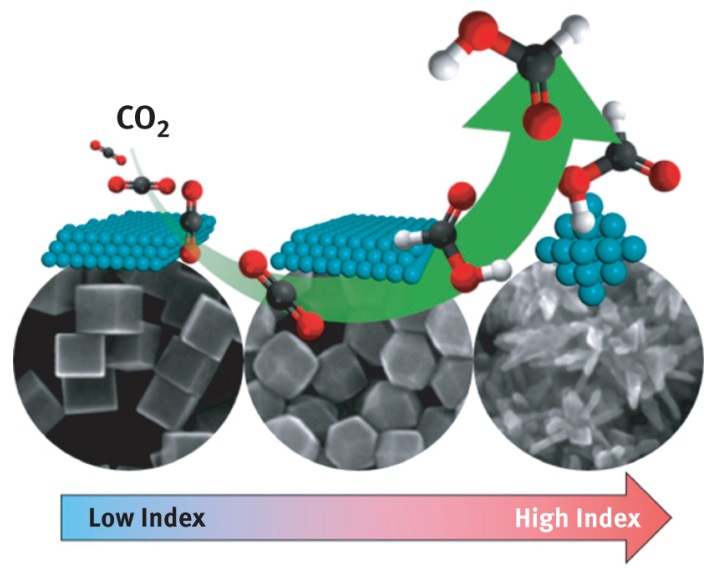

Figure 20: Schematic representation displaying the beneficial effect of the abundance of high-index Pd surfaces to maximize the electrocatalytic reduction of $\mathrm{CO}_{2}$ towards formate. Reprinted with permission from Ref [309]. Copyright (2016) American Chemical Society.

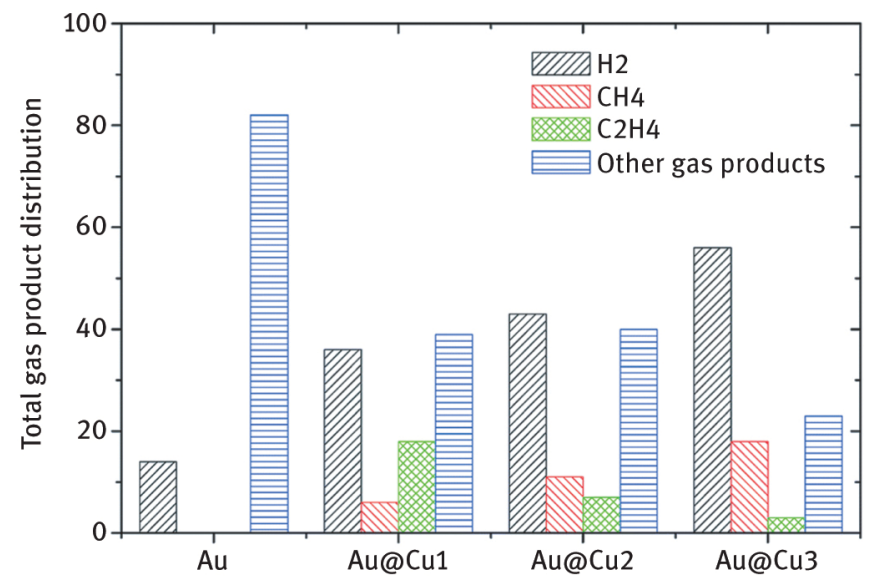

Figure 21: Total gas product distribution of $\mathrm{H}_{2}, \mathrm{CH}_{4}$, and $\mathrm{C}_{2} \mathrm{H}_{4}$ on $\mathrm{Au}$ nanocubes covered with increasing $\mathrm{Cu}$ layers during $\mathrm{CO}_{2}$ electroreduction in PBS $(\mathrm{pH}=8)$ at $-0.6 \mathrm{~V}$ vs. RHE. Reproduced from Ref. [322] with permission from The Royal Society of Chemistry.

Finally, Sun and co-workers evaluated the electrochemical reduction $\mathrm{CO}_{2}$ on $\mathrm{Cu}$ decorated THH and $\{111\}-$ faceted Pd nanoparticles [324]. In this case, both the $\mathrm{Cu}$ coverage and the surface structure of the substrate strongly determined the resulting selectivity towards methanol and ethanol.

For those readers particularly interested in this reaction, we refer to some of the recent and excellent reviews widely covering this topic [150, 277, 325-327].

\section{Critical safety considerations}

Due to the increasing number of applications using different types of nanomaterials, and in particular metal nanoparticles, a great emphasis is nowadays focused on risk assessment of these nanoscale metal materials. To the best of our knowledge, there is no specific international protocols dealing with the possible risks of nanoparticles and safety aspects to face during the various stages of preparation, manipulation, and use. However, relevant contributions have been already reported on the literature about the potential toxicological effects associated with the employment of nanomaterials in human environments [328-330] and in agriculture and food [331].

Additionally, some safety considerations for the employ of nanomaterials have been also reported [332-336].

Very recently, Jeevanandam et al. have discussed about the types of toxic reactions associated with different types of nanomaterials and the regulations implemented by different countries to reduce the associated risks [337].

It is worth noting that the toxicity degree of these metal nanoparticles is affected by a number of factors including size, shape, agglomeration state, surface functionalization, atomic composition, and dose, among 
others [338-345]. Consequently, their interaction with biological environment and living systems will be determined by their physicochemical properties.

In conclusion, and despite some considerations and recommendations being reported, there is no common international legislation dealing with their manipulation and hazard assessment. More work is still required to properly understand the toxic effects and safety aspects for short- and/or long-term exposition.

\section{Conclusions and future perspective}

Understanding the correlations between the shape and the surface structure of metal nanoparticles and their resulting electrocatalytic properties necessarily requires the use of clean nanoparticles. In this contribution, we have reviewed and discussed the most significant protocols and procedures for producing clean shaped metal nanoparticles. In addition, a section focused on the employ of easy-to-do electrochemical experiments to evaluate the cleanness of the nanoparticles have been also included. Interestingly, the use of electrochemical surface structure-sensitive reaction allows obtaining a detailed characterization of the surface structure of the shaped nanoparticles both from qualitative and quantitative points of view. Finally, a collection of relevant studies on the use of different shaped metal nanoparticles for relevant electrochemical reaction including electrooxidation of formic acid, methanol and ethanol, and electroreduction of oxygen and $\mathrm{CO}_{2}$ have been also reported.

Despite the significant improvements due to the use of shaped metal nanoparticle in the field of the Electrocatalysis, there are still a number of issues waiting to be explored including, (i) systematic long-term stability measurements, particularly in-operando conditions, (ii) selectivity issues still need more systematic studies, and (iii) the incorporation of these shaped metal electrocatalysts on practical electrochemical devices such as fuel cells, electrolysers, or filter press-type reactors is still scarce.

\section{Funding}

The authors acknowledge financial support from the Spanish Ministry of Economy and Competitiveness (MINECO) (project CTQ2016-76231-C2-2-R (AEI/FEDER, UE)). J.S-G. also acknowledges financial support from VITC (Vicerrectorado de Investigación y Transferencia de Conocimiento) of the University of Alicante (UATALENTO16-02).

\section{References}

[1] Wieckowski A. Interfacial electrochemistry: theory, experiment, and applications. New York: Marcel Dekker, 1999.

[2] Climent V, Feliu JM. Thirty years of platinum single crystal electrochemistry. J Solid State Electrochem. 2011;15:1297-315.

[3] Martínez-Hincapié R, Sebastián-Pascual P, Climent V, Feliu JM. Investigating interfacial parameters with platinum single crystal electrodes. Russ ] Electrochem. 2017;53:227-36.

[4] Wieckowski A, Savinova ER, Vayenas CG. Catalysis and electrocatalysis at nanoparticle surfaces. New York: CRC Press, 2003.

[5] Koper MT. Fuel cell catalysis: a surface science approach. electrocatalysis and electrochemistry. Hoboken, New Jersey: John Wiley \& Sons, 2009.

[6] Koper MT. Structure sensitivity and nanoscale effects in electrocatalysis. Nanoscale. 2001;3:2054-73.

[7] Solla-Cullón ], Vidal-Iglesias F], Feliu JM. Shape dependent electrocatalysis, annual reports on the progress of chemistry. Section C: Phys Chem. 2011;107:263-97.

[8] Tian N, Zhou ZY, Sun SC. Platinum metal catalysts of high-index surfaces: from single-crystal planes to electrochemically shapecontrolled nanoparticles. J Phys Chem C. 2008;112:19801-17.

[9] Zhou Z-Y, Tian N, Huang -Z-Z, Chen D-J, Sun S-G. Nanoparticle catalysts with high energy surfaces and enhanced activity synthesized by electrochemical method. Faraday Discuss. 2009;140:81-92.

[10] Proussevitch AA, Sahagian DL. Recognition and separation of discrete objects within complex 3D voxelized structures. Comput Ceosci. 2001;27:441-54.

[11] Baletto F, Ferrando R. Structural properties of nanoclusters: energetic, thermodynamic, and kinetic effects. Rev Mod Phys. 2005;77:371423.

[12] Wang T, Lee C, Schmidt LD. Shape and orientation of supported platinum particles. Surf Sci. 1985;163:181-97.

[13] Harris P]. Sulphur-induced faceting of platinum catalyst particles. Nature. 1986;323:792-4.

[14] Ahmadi TS, Wang ZL, Green TC, Henglein A, El-Sayed MA. Shape-controlled synthesis of colloidal platinum nanoparticles. Science. 1996;272:1924-6.

[15] Burda C, Chen X, Narayanan R, El-Sayed MA. Chemistry and properties of nanocrystals of different shapes. Chem Rev. 2005;105:1025102. (Washington, DC, United States).

[16] Tao AR, Habas S, Yang P. Shape control of colloidal metal nanocrystals. Small. 2008;4:310-25. 
[17] Xia Y, Xiong Y, Lim B, Skrabalak SE. Shape-controlled synthesis of metal nanocrystals: simple chemistry meets complex physics? Angew Chem Int Ed. 2009;48:60-103.

[18] Sau K, Rogach AL. Non-spherical noble metal nanoparticles: colloid-chemical synthesis and morphology control. Adv Mater. 2010;22:1781-804. (Weinheim, Germany).

[19] Gu ], Zhang YW, Tao F. Shape control of bimetallic nanocatalysts through well-designed colloidal chemistry approaches. Chem Soc Rev. 2012;41:8050-65.

[20] You H, Yang S, Ding B, Yang H. Synthesis of colloidal metal and metal alloy nanoparticles for electrochemical energy applications. Chem Soc Rev. 2013;42:2880-904.

[21] Quan Z, Wang Y, Fang ]. High-index faceted noble metal nanocrystals. Acc Chem Res. 2013;46:191-202.

[22] Leong G], Schulze MC, Strand MB, Maloney D, Frisco SL, Dinh HN, et al. Shape-directed platinum nanoparticle synthesis: nanoscale design of novel catalysts. Appl Organomet Chem. 2014;28:1-17.

[23] Xia Y, Xia X, Peng HC. Shape-controlled synthesis of colloidal metal nanocrystals: thermodynamic versus kinetic products. ] Am Chem Soc. 2015;137:7947-66.

[24] Gilroy KD, Ruditskiy A, Peng HC, Qin D, Xia Y. Bimetallic nanocrystals: syntheses, properties, and applications. Chem Rev. 2016;116:10414-72.

[25] Chen Q, Jia Y, Xie S, Xie Z. Well-faceted noble-metal nanocrystals with nonconvex polyhedral shapes. Chem Soc Rev. 2016;45:3207-20.

[26] Yang TH, Gilroy KD, Xia Y. Reduction rate as a quantitative knob for achieving deterministic synthesis of colloidal metal nanocrystals. Chem Sci. 2017;8:6730-49.

[27] Xia Y, Gilroy KD, Peng H-C, Xia X. Seed-mediated growth of colloidal metal nanocrystals. Angew Chem Int Ed. 2017;56:60-95.

[28] Gilroy KD, Yang X, Xie S, Zhao M, Qin D, Xia Y. Shape-controlled synthesis of colloidal metal nanocrystals by replicating the surface atomic structure on the seed. Adv Mater. 2018;1706312. DOI: 10.1002/adma.201706312

[29] Tian N, Zhou Z-Y, Sun S-G, Ding Y, Wang ZL. Synthesis of tetrahexahedral platinum nanocrystals with high-index facets and high electro-oxidation activity. Science. 2007;316:732-35.

[30] Tian N, Xiao ], Zhou Z-Y, Liu H-X, Deng Y-], Huang L, et al. Pt-group bimetallic nanocrystals with high-index facets as high performance electrocatalysts. Faraday Discuss. 2013;162:77-89.

[31] Wei L, Tian N, Zhou ZY, Sun SC. Electrochemically shape-controlled nanoparticles in advances in electrochemical science and engineering:nanopatterned and nanoparticle-modified electrode. In: Alkire RC, Bartlett PN, Lipkowski ], editors. Wiley-VCH, 2017:59-95.

[32] Carpenter MK, Moylan TE, Kukreja RS, Atwan MH, Tessema MM. Solvothermal synthesis of platinum alloy nanoparticles for oxygen reduction electrocatalysis. J Am Chem Soc. 2012;134:8535-42.

[33] Cui C, Gan L, Li HH, Yu SH, Heggen M, Strasser P. Octahedral PtNi nanoparticle catalysts: exceptional oxygen reduction activity by tuning the alloy particle surface composition. Nano Lett. 2012;12:5885-9.

[34] Cumeci C, Marathe A, Behrens RL, Chaudhuri ], Korzeniewski C. Solvothermal synthesis and electrochemical characterization of shapecontrolled Pt nanocrystals. J Phys Chem C. 2014;118:14433-40.

[35] Zhang N, Tsao KC, Pan YT, Yang H. Control of the composition of Pt-ni electrocatalysts in surfactant-free synthesis using neat Nformylpiperidine. Nanoscale. 2016;8:2548-53.

[36] Duca M, Rodriguez P, Yanson Al, Koper MT. Selective electrocatalysis on platinum nanoparticles with preferential (100) orientation prepared by cathodic corrosion. Top Catal. 2014;57:255-64.

[37] Zhang C, Hwang SY, Trout A, Peng Z. Solid-state chemistry-enabled scalable production of octahedral Pt-Ni alloy electrocatalyst for oxygen reduction reaction. J Am Chem Soc. 2014;136:7805-8.

[38] Choi J, Jang JH, Roh CW, Yang S, Kim J, Lim J, et al. Gram-scale synthesis of highly active and durable octahedral PtNi nanoparticle catalysts for proton exchange membrane fuel cell. Appl Catal B: Environ. 2018;225:530-7.

[39] Clavilier ], Faure R, Guinet G, Durand R. Preparation of monocrystalline Pt microelectrodes and electrochemical study of the plane surfaces cut in the direction of the $\{111\}$ and $\{110\}$ planes. J Electroanalytical Chem. 1980;107:205-9.

[40] Clavilier ]. Flame-annealing and cleaning technique in interfacial electrochemistry. Interfacial Electrochemistry: Theory: Experiment, and Applications. In: Wieckowski A, editor. Marcel Dekker, 1999:231-48.

[41] Montiel MA, Vidal-Iglesias F], Montiel V, Solla-Cullón ]. Electrocatalysis on shape-controlled metal nanoparticles: progress in surface cleaning methodologies. Curr Opin Electrochem. 2017;1:34-9.

[42] Climent V, Feliu JM. Surface electrochemistry with Pt single-crystal electrodes. In: Alkire RC, Bartlett PN, Lipkowski ], editors. Advances in electrochemical science and engineering: nanopatternedand nanoparticle-modified electrode. Wiley-VCH, 2017:1-57.

[43] Hoshi N, Kagaya K, Hori Y. Voltammograms of the single-crystal electrodes of palladium in aqueous sulfuric acid electrolyte: pd(S)$[\mathrm{n}(111) \times(111)]$ and $\mathrm{Pd}(\mathrm{S})-[\mathrm{n}(100) \times(111)]$. ] Electroanalytical Chem. 2000;485:55-60.

[44] Hoshi N, Kuroda M, Hori Y. Voltammograms of stepped and kinked stepped surfaces of palladium: $\mathrm{pd}(\mathrm{S})-[\mathrm{n}(111) \times(100)]$ and $\mathrm{Pd}(\mathrm{S})-$ [n(100)×(110)]. J Electroanalytical Chem. 2002;521:155-60.

[45] Hara M, Linke U, Wandlowski T. Preparation and electrochemical characterization of palladium single crystal electrodes in $0.1 \mathrm{M} \mathrm{H}_{2} \mathrm{SO}_{4}$ and $\mathrm{HClO}_{4}$ part I.Low-index phases. Electrochim Acta. 2007;52:5733-48.

[46] Vidal-Iglesias F], Arán-Ais RM, Solla-Cullon ], Herrero E, Feliu ]M. Electrochemical characterization of shape-controlled pt nanoparticles in different supporting electrolytes. ACS Catal. 2012;2:901-10.

[47] Susut C, Chapman CB, Samjeské G, Osawa M, Tong Y. An unexpected enhancement in methanol electro-oxidation on an ensemble of Pt(111) nanofacets: A case of nanoscale single crystal ensemble electrocatalysis. Phys Chem Chem Phys. 2008;10:3712-21.

[48] Van Der Vliet DF, Wang C, Li D, Paulikas AP, Greeley ], Rankin RB, et al. Unique electrochemical adsorption properties of Pt-skin surfaces. Angew Chem Int Ed. 2012;51:3139-42.

[49] Shao M, Odell ]H, Choi SI, Xia Y. Electrochemical surface area measurements of platinum- and palladium-based nanoparticles. Electrochem commun. 2013;31:46-8.

[50] Rudi S, Cui C, Gan L, Strasser P. Comparative study of the electrocatalytically active surface areas (ECSAs) of Pt alloy nanoparticles evaluated by hupd and CO-stripping voltammetry. Electrocatalysis. 2014;5:408-18. 
[51] Hamelin A. Cyclic voltammetry at gold single-crystal surfaces 0.1. Behaviour at low-index faces. ] Electroanalytical Chem. 1996;407:1-11.

[52] Hamelin A. Cyclic voltammetry at gold single-crystal surfaces 0.2. Behaviour of high-index faces. J Electroanalytical Chem. 1996;407:1321.

[53] Ke F-S, Solomon B, Ding Y, Xu G-L, Sun S-G, Wang ZL, et al. Enhanced electrocatalytic activity on gold nanocrystals enclosed by highindex facets for oxygen reduction. Nano Energy. 2014;7:179-88.

[54] Yang S, Lee H. Atomically dispersed platinum on gold nano-octahedra with high catalytic activity on formic acid oxidation. ACS Catal. 2013;3:437-43.

[55] Monzó ], Malewski Y, Vidal-Iglesias F], Solla-Gullón ], Rodriguez P. Electrochemical oxidation of small organic molecules on Au nanoparticles with preferential surface orientation. Chem Electro Chem. 2015;2:958-62.

[56] Nichols R], Magnussen OM, Hotlos ], Twomey T, Behm R], Kolb DM. An in-situ STM study of potential-induced changes in the surface topography of $\mathrm{Au}(100)$ electrodes. J Electroanalytical Chem. 1990;290:21-31.

[57] Schneeweiss MA, Kolb DM. Oxide formation on Au(111) - An in situ STM study. Solid State lonics. 1997;94:171-9.

[58] Itaya K, Sugawara S, Sashikata K, Furuya N. In situ scanning tunneling microscopy of platinum (111) surface with the observation of monatomic steps. J Vac Sci Technol, A-Vacuum Surf Films. 1990;8:515-9.

[59] Hamelin A. Lead adsorption on gold single crystal stepped surfaces. ] Electroanalytical Chem. 1979;101:285-90.

[60] Hamelin A, Katayama A. Lead underpotential deposition on gold single-crystal surfaces: the (100) face and its vicinal faces. ] Electroanalytical Chem. 1981;117:221-32.

[61] Hamelin A. Underpotential deposition of lead on single crystal faces of gold. Part I. The influence of crystallographic orientation of the substrate. J Electroanalytical Chem. 1984;165:167-80.

[62] Hamelin A, Lipkowski ]. Underpotential deposition of lead on gold single crystal faces. Part II. Ceneral discussion. ] Electroanalytical Chem. 1984;171:317-30.

[63] Hernández ], Solla-Gullón ], Herrero E. Cold nanoparticles synthesized in a water-in-oil microemulsion: electrochemical characterization and effect of the surface structure on the oxygen reduction reaction. J Electroanalytical Chem. 2004;574:185-96.

[64] Hernández ], Solla-Gullón ], Herrero E, Aldaz A, Feliu JM. Electrochemistry of shape-controlled catalysts: oxygen reduction reaction on cubic gold nanoparticles. J Phys Chem C. 2007;111:14078-83.

[65] Hernández ], Solla-Gullón ], Herrero E, Feliu JM, Aldaz A. In situ surface characterization and oxygen reduction reaction on shapecontrolled gold nanoparticles. ] Nanosci Nanotechnol. 2009;9:2256-73.

[66] Chierchie T, Mayer C. Voltammetric study of the underpotential deposition of copper on polycrystalline and single crystal palladium surfaces. Electrochim Acta. 1988;33:341-5.

[67] Cuesta A, Kibler LA, Kolb DM. A method to prepare single crystal electrodes of reactive metals: application to Pd(hkl). ] Electroanalytical Chem. 1999;466:165-8.

[68] Fang LL, Tao Q, Li MF, Liao LW, Chen D, Chen YX. Determination of the real surface area of palladium electrode. Chin ] Chem Phys. 2010;23:543-8.

[69] Francke R, Climent V, Baltruschat H, Feliu JM. Electrochemical deposition of copper on stepped platinum surfaces in the 01 (1)overbarzone vicinal to the (100) plane. J Electroanalytical Chem. 2008;624:228-40.

[70] Danilov AI, Molodkina EB, Rudnev AV, Polukarov YM, Feliu JM. Kinetics of copper deposition on Pt(111) and Au(111) electrodes in solutions of different acidities. Electrochim Acta. 2005;50:5032-43.

[71] Vidal-Iglesias F], Solla-Cullón ], Rodríguez P, Herrero E, Montiel V, Feliu JM, et al. Shape-dependent electrocatalysis: ammonia oxidation on platinum nanoparticles with preferential (100) surfaces. Electrochem commun. 2004;6:1080-4.

[72] Solla-Cullón ], Vidal-Iglesias F], Rodríguez P, Herrero E, Feliu JM, Clavilier ], et al. In situ surface characterization of preferentially oriented platinum nanoparticles by using electrochemical structure sensitive adsorption reactions.] Phys Chem B. 2004;108:13573-5.

[73] Erikson H, Sarapuu A, Tammeveski K, Solla-Cullón ], Feliu JM. Enhanced electrocatalytic activity of cubic Pd nanoparticles towards the oxygen reduction reaction in acid media. Electrochem commun. 2011;13:734-7.

[74] Erikson H, Sarapuu A, Alexeyeva N, Tammeveski K, Solla-Cullón ], Feliu JM. Electrochemical reduction of oxygen on palladium nanocubes in acid and alkaline solutions. Electrochim Acta. 2012;59:329-35.

[75] Vidal-Iglesias F], Arán-Ais RM, Solla-Gullon ], Garnier E, Herrero E, Aldaz A, et al. Shape-dependent electrocatalysis: formic acid electrooxidation on cubic Pd nanoparticles. Phys Chem Chem Phys. 2012;14:10258-65.

[76] Coutanceau C, Urchaga P, Brimaud S, Baranton S. Colloidal syntheses of shape- and size-controlled Pt nanoparticles for electrocatalysis. Electrocatalysis. 2012;3:75-87.

[77] Urchaga P, Baranton S, Napporn TW, Coutanceau C. Selective syntheses and electrochemical characterization of platinum nanocubes and nanotetrahedrons/octahedrons. Electrocatalysis. 2010;1:3-6.

[78] Solla-Cullón ], Vidal-Iglesias F], López-Cudero A, Garnier E, Feliu JM, Aldaz A. Shape-dependent electrocatalysis: methanol and formic acid electrooxidation on preferentially oriented Pt nanoparticles. Phys Chem Chem Phys. 2008;10:3689-98.

[79] Brimaud S, Jusys Z, Behm R]. Controlled surface structure for in situ ATR-FTIRS studies using preferentially shaped Pt nanocrystals. Electrocatalysis. 2011;2:69-74.

[80] Brimaud S, Jusys Z, Behm R]. Shape-selected nanocrystals for in situ spectro-electrochemistry studies on structurally well-defined surfaces under controlled electrolyte transport: A combined in situ ATR-FTIR/online DEMS investigation of CO electrooxidation on Pt, Beilstein. J Nanotechnol. 2014;5:735-46.

[81] Susut C, Nguyen TD, Chapman GB, Tong Y. Shape and size stability of Pt nanoparticles for MeOH electro-oxidation. Electrochim Acta. 2008;53:6135-42.

[82] Susut C, Tong Y. Size-dependent methanol electro-oxidation activity of Pt nanoparticles with different shapes. Electrocatalysis. 2011;2:75-81.

[83] Levendorf A, Sun SC, Tong Y. In situ FT-IR investigation of methanol and CO electrooxidation on cubic and octahedral/tetrahedral Pt nanoparticles having residual PVP. Electrocatalysis. 2014;5:248-55. 
[84] Koczkur KM, Mourdikoudis S, Polavarapu L, Skrabalak SE. Polyvinylpyrrolidone (PVP) in nanoparticle synthesis. Dalton Trans. 2015;44:17883-905.

[85] Monzó ], Koper MT, Rodriguez P. Removing polyvinylpyrrolidone from catalytic Pt nanoparticles without modification of superficial order. Chem Phys Chem. 2012;13:709-15.

[86] Levendorf AM, Chen D-], Rom CL, Liu Y, Tong Y]. Electrochemical and in situ ATR-SEIRAS investigations of methanol and CO electrooxidation on PVP-free cubic and octahedral/tetrahedral Pt nanoparticles. RSC Adv. 2014;4:21284-93.

[87] Yang H, Tang Y, Zou S. Electrochemical removal of surfactants from Pt nanocubes. Electrochem commun. 2014;38:134-7.

[88] Zalineeva A, Baranton S, Coutanceau C. Bi-modified palladium nanocubes for glycerol electrooxidation. Electrochem commun. 2013;34:335-8.

[89] Zalineeva A, Baranton S, Coutanceau C, Jerkiewicz G. Electrochemical behavior of unsupported shaped palladium nanoparticles. Langmuir. 2015;31:1605-9.

[90] Nalajala N, Gooty Saleha WF, Ladewig BP, Neergat M. Sodium borohydride treatment: A simple and effective process for the removal of stabilizer and capping agents from shape-controlled palladium nanoparticles. Chem Commun. 2014;50:9365-8.

[91] Naresh N, Wasim FC, Ladewig BP, Neergat M. Removal of surfactant and capping agent from Pd nanocubes (Pd-NCs) using tertbutylamine: its effect on electrochemical characteristics. J Mater Chem. 2013;1:8553-9.

[92] Luo M, Hong Y, Yao W, Huang C, Xu Q, Wu Q. Facile removal of polyvinylpyrrolidone (PVP) adsorbates from Pt alloy nanoparticles. ] Mater Chem. 2015;3:2770-5.

[93] Arán-Ais RM, Vidal-Iglesias F], Solla-Cullon ], Herrero E, Feliu JM. Electrochemical characterization of clean shape-controlled Pt nanoparticles prepared in presence of oleylamine/oleic acid. Electroanalysis. 2014;27:945-56.

[94] Safo IA, Oezaslan M. Electrochemical Cleaning of Polyvinylpyrrolidone-capped Pt Nanocubes for the Oxygen Reduction Reaction. Electrochim Acta. 2017;241:544-52.

[95] Safo IA, Dosche C, Oezaslan M. TEM, FTIR and Electrochemistry Study: desorption of PVP from Pt Nanocubes. Zeitschrift für Physikalische Chemie. 2018. DOI: 10.1515/zpch-2018-1147

[96] Hernández ], Solla-Cullón ], Herrero E, Aldaz A, Feliu JM. Characterization of the surface structure of gold nanoparticles and nanorods using structure sensitive reactions. J Phys Chem B. 2005;109:12651-4.

[97] Chen Y, Milenkovic S, Hassel AW. \{110\}-Terminated square-shaped gold nanoplates and their electrochemical surface reactivity. Chem Electro Chem. 2017;4:557-64.

[98] Yang S, Park N-Y, Han JW, Kim C, Lee S-C, Lee H. A distinct platinum growth mode on shaped gold nanocrystals. Chem Commun. 2012;48:257-9.

[99] Solla-Cullón ], Rodríguez P, Herrero E, Aldaz A, Feliu JM. Surface characterization of platinum electrodes. Phys Chem Chem Phys. 2008;10:1359-73.

[100] Solla-Cullón ], Vidal-Iglesias F], Herrero E, Feliu JM, Aldaz A. Electrocatalysis on shape-controlled Pt nanoparticles in polymer electrolyte fuel cells: science, applications and challenges. Polymer Electrolyte Fuel Cells: Science, Applications, and Challenges. In: Franco AA, editor. Pan Stanford, 2013:93-151.

[101] Arán-Ais RM, Solla-Cullón ], Herrero E, Feliu JM. On the quality and stability of preferentially oriented (100) Pt nanoparticles: an electrochemical insight. ] Electroanalytical Chem. 2018;808:433-8.

[102] Vidal-Iglesias F], Solla-Cullón ], Herrero E, Montiel V, Aldaz A, Feliu JM. Evaluating the ozone cleaning treatment in shape-controlled Pt nanoparticles: evidences of atomic surface disordering. Electrochem commun. 2011;13:502-5.

[103] Park JY, Aliaga C, Renzas ]R, Lee H, Somorjai CA. The role of organic capping layers of platinum nanoparticles in catalytic activity of CO oxidation. Catal Lett. 2009;129:1-6.

[104] Aliaga C, Park JY, Yamada Y, Lee HS, Tsung CK, Yang P, et al. Sum frequency generation and catalytic reaction studies of the removal of organic capping agents from Pt nanoparticles by UV-Ozone treatment. J Phys Chem C. 2009;113:6150-5.

[105] Krier ]M, Michalak WD, Baker LR, An K, Komvopoulos K, Somorjai CA. Sum frequency generation vibrational spectroscopy of colloidal platinum nanoparticle catalysts: disordering versus removal of organic capping. J Phys Chem C. 2012;116:17540-6.

[106] Crespo-Quesada M, Andanson JM, Yarulin A, Lim B, Xia Y, Kiwi-Minsker L. UV-ozone cleaning of supported poly(vinylpyrrolidone)stabilized palladium nanocubes: effect of stabilizer removal on morphology and catalytic behaviour. Langmuir. 2011;27:7909-16.

[107] Herrero E, Buller L], Abruña HD. Underpotential deposition at single crystal surfaces of Au, Pt, Ag and other materials. Chem Rev. 2001;101:1897-930. (Washington, DC, United States).

[108] Oviedo OA, Vélez P, Macagno VA, Leiva EP. Underpotential deposition: from planar surfaces to nanoparticles. Surf Sci. 2015;631:22-34.

[109] Jiang Q, Jiang Z, Zhang L, Lin H, Yang N, Li H, et al. Synthesis and high electrocatalytic performance of hexagram shaped gold particles having an open surface structure with kinks. Nano Res. 2011;4:612-22.

[110] Hebié S, Kokoh KB, Servat K, Napporn TW. Shape-dependent electrocatalytic activity of free gold nanoparticles toward glucose oxidation. Gold Bulletin. 2013;46:311-8.

[111] Jeyabharathi C, Zander M, Scholz F. Underpotential deposition of lead on quasi-spherical and faceted gold nanoparticles. ] Electroanalytical Chem. 2018. DOI: 10.1016/j.jelechem.2017.10.011

[112] Jin M, Zhang H, Xie Z, Xia Y. Palladium nanocrystals enclosed by $\{100\}$ and $\{111\}$ facets in controlled proportions and their catalytic activities for formic acid oxidation. Energy Environ Sci. 2012;5:6352-7.

[113] Solla-Cullón ], Carnier E, Feliu JM, Leoni M, Leonardi A, Scardi P. Structure and morphology of shape-controlled Pd nanocrystals. ] of. Appl Crystallogr. 2015;48:1534-42.

[114] Higuchi E, Kawai M, Chiku M, Inoue H. Synthesis and electrochemical characterization of palladium crystals enclosed by (100) facets by seed-mediated fabrication. Int ] Electrochem. 2018. Article ID 7138638. DOI: 10.1155/2018/7138638.

[115] Rodríguez P, Solla-Cullón J, Vidal-Iglesias F], Herrero E, Aldaz A, Feliu JM. Determination of (111) ordered domains on platinum electrodes by irreversible adsorption of bismuth. Anal Chem. 2005;77:5317-23.

[116] Rodríguez P, Herrero E, Solla-Gullón ], Vidal-Iglesias F], Aldaz A, Feliu JM. Specific surface reactions for identification of platinum surface domains - Surface characterization and electrocatalytic tests. Electrochim Acta. 2005;50:4308-17. 
[117] Rodríguez P, Herrero E, Aldaz A, Feliu JM. Tellurium adatoms as an in-situ surface probe of (111) two-dimensional domains at platinum surfaces. Langmuir. 2006;22:10329-37.

[118] Rodríguez P, Herrero E, Solla-Cullón ], Vidal-Iglesias F], Aldaz A, Feliu JM. Electrochemical characterization of irreversibly adsorbed germanium on platinum stepped surfaces vicinal to Pt(100). Electrochim Acta. 2005;50:3111-21.

[119] Brimaud S, Pronier S, Coutanceau C, Léger ]M. New findings on CO electrooxidation at platinum nanoparticle surfaces. Electrochem commun. 2008;10:1703-7.

[120] Devivaraprasad R, Ramesh R, Naresh N, Kar T, Singh RK, Neergat M. Oxygen reduction reaction and peroxide generation on shapecontrolled and polycrystalline platinum nanoparticles in acidic and alkaline electrolytes. Langmuir. 2014;30:8995-9006.

[121] Bertin E, Garbarino S, Guay D. Formic acid oxidation on Bi covered Pt electrodeposited thin films: influence of the underlying structure. Electrochim Acta. 2014;134:486-95.

[122] Devivaraprasad R, Kar T, Chakraborty A, Singh RK, Neergat M. Reconstruction and dissolution of shape-controlled Pt nanoparticles in acidic electrolytes. Phys Chem Chem Phys. 2016;18:11220-32.

[123] Liu ], Fan X, Liu X, Song Z, Deng Y, Han X, et al. Synthesis of cubic-shaped Pt particles with (100) preferential orientation by a quick, onestep and clean electrochemical method. ACS Appl Mater Interfaces. 2017;9:18856-64.

[124] Liu Z, Ma C, Liu ], Chen X, Song Z, Hu W, et al. Studies on the electrochemical stability of preferentially (100)-oriented Pt prepared through three different methods. Chem Electro Chem. 2017;4:66-74.

[125] Rodriguez-Lopez M, Solla-Gullón ], Herrero E, Tuñon P, Feliu ]M, Aldaz A, et al. Electrochemical reactivity of aromatic molecules at nanometer-sized surface domains: from $\mathrm{Pt}(\mathrm{hkl})$ single crystal electrodes to preferentially oriented platinum nanoparticles. J Am Chem Soc. 2010;132:2233-42.

[126] Rodríguez P, Herrero E, Solla-Cullón ], Feliu ]M, Aldaz A. Selective electrocatalysis of acetaldehyde oxime reduction on (111) sites of platinum single crystal electrodes and nanoparticles surfaces. J Solid State Electrochem. 2008;12:575-81.

[127] Martínez-Rodríguez RA, Vidal-Iglesias F], Solla-Cullón ], Cabrera CR, Feliu JM. synthesis and electrocatalytic properties of $\mathrm{H}_{2} \mathrm{SO}_{4}$ induced (100) Pt nanoparticles prepared in water-in-oil microemulsion. Chem Phys Chem. 2014;15:1997-2001.

[128] El-Deab MS. On the preferential crystallographic orientation of Au nanoparticles: effect of electrodeposition time. Electrochim Acta. 2009;54:3720-5.

[129] El-Deab MS, Sotomura T, Ohsaka T. Size and crystallographic orientation controls of gold nanoparticles electrodeposited on CC electrodes. J Electrochem Soc. 2005;152:C1-C6.

[130] El-Deab MS, Arihara K, Ohsaka T. Fabrication of Au(111)-like polycrystalline gold electrodes and their applications to oxygen reduction. ] Electrochem Soc. 2004;151:E213-E218.

[131] Arihara K, Ariga T, Takashima N, Arihara K, Okajima T, Kitamura F, et al. Multiple voltammetric waves for reductive desorption of cysteine and 4-mercaptobenzoic acid monolayers self-assembled on gold substrates. Phys Chem Chem Phys. 2003;5:3758-61.

[132] Cuesta A, Kibler LA, Kolb DM. A method to prepare single crystal electrodes of reactive metals: application to Pd(hkl). ] Electroanalytical Chem. 1999;46:165-8.

[133] Vidal-Iglesias F], Al-Akl A, Watson D], Attard CA. A new method for the preparation of PtPd alloy single crystal surfaces. Electrochem commun. 2006;8:1147-50.

[134] Moniri S, Cleve TV, Linic S. Pitfalls and best practices in measurements of the electrochemical surface area of platinum-based nanostructured electro-catalysts. ] Catal. 2017;345:1-10.

[135] Vidal-Iglesias F], García-Aráez N, Montiel V, Feliu JM, Aldaz A. Selective electrocatalysis of ammonia oxidation on Pt (1 0 0) sites in alkaline medium. Electrochem Commun. 2003;5:22-6.

[136] Vidal-Iglesias F], Solla-Gullón ], Montiel V, Feliu JM, Aldaz A. Ammonia selective oxidation on Pt (100) sites in an alkaline medium. ] Phys Chem B. 2005;109:12914-9.

[137] Peng Z, Yang H. Designer platinum nanoparticles: control of shape, composition in alloy, nanostructure and electrocatalytic property. Nano Today. 2009;4:143-64.

[138] Chen ], Lim B, Lee EP, Xia Y. Shape-controlled synthesis of platinum nanocrystals for catalytic and electrocatalytic applications. Nano Today. 2009;4:81-95.

[139] Bing Y, Liu H, Zhang L, Chosh D, Zhang ]. Nanostructured Pt-alloy electrocatalysts for PEM fuel cell oxygen reduction reaction. Chem Soc Rev. 2010;39:2184-202.

[140] Wu B, Zheng N. Surface and interface control of noble metal nanocrystals for catalytic and electrocatalytic applications. Nano Today. 2013;8:168-97.

[141] Sanchez-Sanchez CM, Solla-Cullon ], Montiel V. Electrocatalysis at nanoparticles in Electrochemistry: nanosystems Electrochemistry. Royal Soc Chem. 2013;11:34-70.

[142] Kleijn SE, Lai SC, Koper MT, Unwin PR. Electrochemistry of nanoparticles. Angew Chem Int Ed. 2014;53:3558-86.

[143] Wang Y], Zhao N, Fang B, Li H, Bi XT, Wang H. Carbon-supported Pt-based alloy electrocatalysts for the oxygen reduction reaction in polymer electrolyte membrane fuel cells: particle size, shape, and composition manipulation and their impact to activity. Chem Rev. 2015;115:3433-67.

[144] Vidal-Iglesias F], Solla-Cullón ], Feliu JM. Recent advances in the use of shape-controlled metal nanoparticles in electrocatalysis. In Ozoemena IK, Chen S, editors. Nanomaterials for fuel cell catalysis. Springer International Publishing, 2016:31-92.

[145] Cao S, Tao FF, Tang Y, Li Y, Yu ]. Size-and shape-dependent catalytic performances of oxidation and reduction reactions on nanocatalysts. Chem Soc Rev. 2016;45:4747-65.

[146] Kang Y, Yang P, Markovic NM, Stamenkovic VR. Shaping electrocatalysis through tailored nanomaterials. Nano Today. 2016;11:587-600.

[147] Hong JW, Kim Y, Kwon Y, Han SW. Noble metal nanocrystals with controlled facets for electrocatalysis. Chem: Asian ]. 2016;11:2224-39.

[148] Liu X, Li W, Zou S. Electrocatalysis of Facet-controlled Noble Metal Nanomaterials for Low-temperature Fuel Cells. In: Maiyalagan T, Saji VS, editors. Electrocatalysts for low temperature fuel cells: fundamentals and recent trends. Wiley-VCH, 2017:373-99.

[149] Tian N, Lu B-A, Yang X-D, Huang R, Jiang Y-X, Zhou Z-Y, et al. Rational design and synthesis of low-temperature fuel cell electrocatalysts. Electrochem Energy Rev. 2018;1:54-83. 
[150] Strasser P, Cliech M, Kuehl S, Moeller T. Electrochemical processes on solid shaped nanoparticles with defined facets. Chem Soc Rev. 2018;47:715-35.

[151] Shao M. Electrocatalysis in fuel cells: a non- and low- platinum approach. London: Springer, 2013.

[152] Feliu JM, Herrero E. Formic acid oxidation in handbook of fuel cells - Fundamentals, technology and applications, vol. 2. Vielstich W, Gasteiger H, Lamm A editors, John Wiley \& Sons, 2003:625-34.

[153] ]iang K, Zhang HX, Zou S, Cai WB. Electrocatalysis of formic acid on palladium and platinum surfaces: from fundamental mechanisms to fuel cell applications. Phys Chem Chem Phys. 2014;16:20360-76.

[154] Capon A, Parsons R. The oxidation of formic acid at noble metal electrodes: I. Review of previous work. ] Electroanalytical Chem. 1973;44:1-7.

[155] Capon A, Parsons R. The oxidation of formic acid on noble metal electrodes: II. Comp Behav Pure Electrodes ] Electroanal Chem. 1973;44:239-54.

[156] Capon A, Parsons R. The oxidation of formic acid at noble metal electrodes Part III. Intermediates and mechanism on platinum electrodes. J Electroanalytical Chem. 1973;45:205-31.

[157] Grozovski V, Solla-Cullon ], Climent V, Herrero E, Feliu JM. Formic acid oxidation on shape-controlled Pt nanoparticles studied by pulsed voltammetry. ] Phys Chem C. 2010;114:13802-12.

[158] Huang X, Zhao Z, Fan ], Tan Y, Zheng N. Amine-assisted synthesis of concave polyhedral platinum nanocrystals having $\{411\}$ high-index facets. J Am Chem Soc. 2011;133:4718-21.

[159] Li Y, Jiang Y, Chen M, Liao H, Huang R, Zhou Z, et al. Electrochemically shape-controlled synthesis of trapezohedral platinum nanocrystals with high electrocatalytic activity. Chem Commun. 2012;48:9531-3. (Cambridge, United Kingdom).

[160] Xia BY, Wu HB, Wang X, Lou XW. Highly concave platinum nanoframes with high-index facets and enhanced electrocatalytic properties. Angew Chem Int Ed. 2013;52:12337-40.

[161] Lu B-A, Du J-H, Sheng T, Tian N, Xiao ], Liu L, et al. Hydrogen adsorption-mediated synthesis of concave Pt nanocubes and their enhanced electrocatalytic activity. Nanoscale. 2016;8:11559-64.

[162] Rice C, Ha S, Masel RI, Wieckowski A. Catalysts for direct formic acid fuel cells. ] Power Sources. 2003;115:229-35.

[163] Hoshi N, Kida K, Nakamura M, Nakada M, Osada K. Structural effects of electrochemical oxidation of formic acid on single crystal electrodes of palladium. J Phys Chem B. 2006;110:12480-4.

[164] Baldauf M, Kolb DM. Formic acid oxidation on ultrathin Pd films on Au(hkl) and Pt(hkl) electrodes. ] Phys Chem. 1996;100:11375-81.

[165] Zhang H-X, Wang H, Re Y-S, Cai W-B. Palladium nanocrystals bound by $\{110\}$ or $\{100\}$ facets: from one pot synthesis to electrochemistry. Chem Commun. 2012;48:8362-4.

[166] Shao Z, Zhu W, Wang H, Yang Q, Yang S, Liu X, et al. Controllable synthesis of concave nanocubes, right bipyramids, and 5-fold twinned nanorods of palladium and their enhanced electrocatalytic performance. J Phys Chem C. 2013;117:14289-94.

[167] Kuo CH, Lamontagne LK, Brodsky CN, Chou LY, Zhuang ], Sneed BT, et al. The effect of lattice strain on the catalytic properties of Pd nanocrystals. Chem Sus Chem. 2013;6:1993-2000.

[168] Xia X, Choi SI, Herron JA, Lu N, Scaranto ], Peng HC, et al. Facile synthesis of palladium right bipyramids and their use as seeds for overgrowth and as catalysts for formic acid oxidation. J Am Chem Soc. 2013;135:15706-9.

[169] Lv T, Wang Y, Choi SI, Chi M, Tao ], Pan L, et al. Controlled synthesis of nanosized palladium icosahedra and their catalytic activity towards formic-acid oxidation. Chem Sus Chem. 2013;6:1923-30.

[170] Shao M, Odell ], Humbert M, Yu T, Xia Y. Electrocatalysis on shape-controlled palladium nanocrystals: oxygen reduction reaction and formic acid oxidation. J Phys Chem C. 2013;117:4172-80.

[171] Zhang X, Yin H, Wang ], Chang L, Gao Y, Liu W, et al. Shape-dependent electrocatalytic activity of monodispersed palladium nanocrystals toward formic acid oxidation. Nanoscale. 2013;5:8392-7.

[172] Tang Y, Edelmann RE, Zou S. Length tunable penta-twinned palladium nanorods: seedless synthesis and electrooxidation of formic acid. Nanoscale. 2014;6:5630-3.

[173] Chen Q-S, Xu Z-N, Peng S-Y, Chen Y-M, Lv D-M, Wang Z-Q, et al. One-step electrochemical synthesis of preferentially oriented (111) Pd nanocrystals supported on graphene nanoplatelets for formic acid electrooxidation. J Power Sources. 2015;282:471-8.

[174] Zheng W, Qu ], Hong X, Tedsree K, Tsang SC. Probing the size and shape effects of cubic- and spherical-shaped palladium nanoparticles in the electrooxidation of formic acid. Chem Cat Chem. 2015;7:3826-31.

[175] Huang H, Ruditskiy A, Choi S, Zhang L, Liu ], Ye Z, et al. One-pot synthesis of penta-twinned palladium nanowires and their enhanced electrocatalytic properties. ACS Appl Mater Interfaces. 2017;9:31203-12.

[176] Klinkova A, Luna PD, Sargent EH, Kumacheva E, Cherepanov PV. Enhanced electrocatalytic performance of palladium nanoparticles with high energy surfaces in formic acid oxidation. J Mater Chem. 2017;5:11582-5.

[177] Sheng T, Xu Y-F, Jiang Y-X, Huang L, Tian N, Zhou Z-Y, et al. Structure design and performance tuning of nanomaterials for electrochemical energy conversion and storage. Acc Chem Res. 2016;49:2569-77.

[178] Boronat-Conzalez A, Herrero E, Feliu JM. Fundamental aspects of $\mathrm{HCOOH}$ oxidation at platinum single crystal surfaces with basal orientations and modified by irreversibly adsorbed adatoms. J Solid State Electrochem. 2014;18:1181-93.

[179] Nørskov JK, Abild-Pedersen F, Studt F, Bligaard T. Density functional theory in surface chemistry and catalysis. Proc Natl Acad Sci. 2011;108:937-43.

[180] Porter NS, Wu H, Quan Z, Fang ]. Shape-control and electrocatalytic activity-enhancement of Pt-based bimetallic nanocrystals. Acc Chem Res. 2013;46:1867-77.

[181] Yuan Q, Zhou Z, Zhuang ], Wang X. Pd-Pt random alloy nanocubes with tunable compositions and their enhanced electrocatalytic activities. Chem Commun. 2010;46:1491-3.

[182] Zhang ], Yang H, Yang K, Fang ], Zou S, Luo Z, et al. Monodisperse Pt ${ }_{3} F e$ nanocubes: synthesis, characterization, self-assembly, and electrocatalytic activity. Adv Funct Mater. 2010;20:3727-33.

[183] Yang H, Dai L, Xu D, Fang ], Zou S. Electrooxidation of methanol and formic acid on PtCu nanoparticles. Electrochim Acta. 2010;55:8000-4. 
[184] Xu D, Bliznakov S, Liu Z, Fang ], Dimitrov N. Composition-dependent electrocatalytic activity of Pt-Cu nanocube catalysts for formic acid oxidation. Angew Chem Int Ed. 2010;49:1282-5.

[185] Zhang ], Yang H, Martens B, Luo Z, Xu D, Wang Y, et al. Pt-Cu nanoctahedra: synthesis and comparative study with nanocubes on their electrochemical catalytic performance. Chem Sci. 2012;3:3302-6.

[186] Bromberg L, Fayette M, Martens B, Luo ZP, Wang Y, Xu D, et al. Catalytic performance comparison of shape-dependent nanocrystals and oriented ultrathin films of $\mathrm{Pt}_{4} \mathrm{Cu}$ alloy in the formic acid oxidation process. Electrocatalysis. 2013;4:24-36.

[187] Kang Y, Murray CB. Synthesis and electrocatalytic properties of cubic Mn-Pt nanocrystals (nanocubes). ] Am Chem Soc. 2010;132:75689.

[188] Yu Y, Zhang Q, Liu B, Lee JY. Synthesis of nanocrystals with variable high-index Pd facets through the controlled heteroepitaxial growth of trisoctahedral Au templates. J Am Chem Soc. 2010;132:18258-65.

[189] Zhang L, Zhang ], Kuang Q, Xie S, Jiang Z, Xie Z, et al. $\mathrm{Cu}^{2+}$-assisted synthesis of hexoctahedral Au-Pd alloy nanocrystals with high-index facets. J Am Chem Soc. 2011;133:17114-7.

[190] Deng Y], Tian N, Zhou ZY, Huang R, Liu ZL, Xiao ], et al. Alloy tetrahexahedral Pd-Pt catalysts: enhancing significantly the catalytic activity by synergy effect of high-index facets and electronic structure. Chem Sci. 2012;3:1157-61.

[191] Jia Y, Jiang Y, Zhang J, Zhang L, Chen Q, Xie Z, et al. Unique excavated rhombic dodecahedral PtCuz alloy nanocrystals constructed with ultrathin nanosheets of high-energy \{110\} facets. J Am Chem Soc. 2014;136:3748-51.

[192] Sneed BT, Young AP, Jalalpoor D, Golden MC, Mao S, Jiang Y, et al. Shaped Pd-ni-pt core-sandwich-shell nanoparticles: influence of Ni sandwich layers on catalytic electrooxidations. ACS Nano. 2014;8:7239-50.

[193] Zhang L, Choi S-I, Tao ], Peng H-C, Xie S, Zhu Y, et al. Pd-Cu bimetallic tripods: a mechanistic understanding of the synthesis and their enhanced electrocatalytic activity for formic acid oxidation. Adv Funct Mater. 2014;24:7520-9.

[194] López-Cudero A, Vidal-Iglesias F], Solla-Gullón ], Herrero E, Aldaz A, Feliu ]M. Formic acid electrooxidation on Bi-modified polyoriented and preferential (111) Pt nanoparticles. Phys Chem Chem Phys. 2009;11:416-24.

[195] Chen QS, Zhou ZY, Vidal-Iglesias F], Solla-Cullón ], Feliu JM, Sun SG. Significantly enhancing catalytic activity of tetrahexahedral Pt nanocrystals by Bi adatom decoration. J Am Chem Soc. 2011;133:12930-3.

[196] Vidal-Iglesias F], Solla-Gullón ], Herrero E, Aldaz A, Feliu JM. Pd adatom decorated (100) preferentially oriented Pt nanoparticles for formic acid electrooxidation. Angew Chem Int Ed. 2010;49:6998-7001.

[197] Vidal-Iglesias F], Lopez-Cudero A, Solla-Cullón ], Aldaz A, Feliu JM. Pd-modified shape-controlled Pt nanoparticles towards formic acid electrooxidation. Electrocatalysis. 2012;3:313-23.

[198] Vidal-Iglesias F], López-Cudero A, Solla-Cullón ], Feliu JM. Towards more active and stable electrocatalysts for formic acid electrooxidation: antimony-decorated octahedral platinum nanoparticles. Angew Chem Int Ed. 2013;52:964-7.

[199] Busó-Rogero C, Perales-Rondón JV, Farias M], Vidal-Iglesias F], Solla-Gullón ], Herrero E, et al. Formic acid electrooxidation on thalliumdecorated shape-controlled platinum nanoparticles: an improvement in electrocatalytic activity. Phys Chem Chem Phys. 2014;16:1361624.

[200] Perales-Rondón JV, Solla-Cullón ], Herrero E, Sánchez-Sánchez CM. Enhanced catalytic activity and stability for the electrooxidation of formic acid on lead modified shape controlled platinum nanoparticles. Appl Catal B: Environ. 2017;201:48-57.

[201] Liu HX, Tian N, Brandon MP, Pei ], Huangfu ZC, Zhan C, et al. Enhancing the activity and tuning the mechanism of formic acid oxidation at tetrahexahedral Pt nanocrystals by Au decoration. Phys Chem Chem Phys. 2012;14:16415-23.

[202] Li M, Adzic R. Low-platinum-content electrocatalysts for methanol and ethanol electrooxidation. In: Shao M, editor. Electrocatalysis in fuel cells, vol. 9. Lecture Notes in Energy. London: Springer, 2013:1-25.

[203] Lamy C, Leger JM, Clavilier], Parsons R. Structural effects in electrocatalysis: a comparative study of the oxidation of $\mathrm{CO}, \mathrm{HCOOH}$ and $\mathrm{CH}_{3} \mathrm{OH}$ on single crystal Pt electrodes. J Electroanalytical Chem. 1983;150:71-7.

[204] Xia XH, Iwasita T, Ge F, Vielstich W. Structural effects and reactivity in methanol oxidation on polycrystal line and single crystal platinum. Electrochim Acta. 1996;41:711-8.

[205] Housmans TH, Wonders AH, Koper MT. Structure sensitivity of methanol electrooxidation pathways on platinum: an on-line electrochemical mass spectrometry study. J Phys Chem B. 2006;110:10021-31.

[206] Chen ], Mao ], Zhao ], Ren M, Wei M. Surfactant-free platinum nanocubes with greatly enhanced activity towards methanol/ethanol electrooxidation. RSC Adv. 2014;4:28832-5.

[207] Arjona N, Guerra-Balcázar M, Ortiz-Frade L, Osorio-Monreal G, Álvarez-Contreras L, Ledesma-Carcía ], et al. Electrocatalytic activity of well-defined and homogeneous cubic-shaped Pd nanoparticles. J Mater Chem. 2013;1:15524-9.

[208] Kannan P, Maiyalagan T, Opallo M. One-pot synthesis of chain-like palladium nanocubes and their enhanced electrocatalytic activity for fuel-cell applications. Nano Energy. 2013;2:677-87.

[209] Qin Y-L, Zhang X-B, Wang ], Wang L-M. Rapid and shape-controlled synthesis of "clean" star-like and concave Pd nanocrystallites and their high performance toward methanol oxidation. J Mater Chem. 2012;22:14861-3.

[210] Waszczuk P, Solla-Cullon ], Kim H-S, Tong YY, Montiel V, Aldaz A, et al. Methanol electrooxidation on platinum/ruthenium nanoparticle catalysts. J Catal. 2001;203:1-6

[211] Maillard F, Lu G-Q, Wieckowski A, Stimming U. Ru-decorated Pt surfaces as model fuel cell electrocatalysts for CO electrooxidation. ] Phys Chem B. 2005;109:16230-43.

[212] Liu H-X, Tian N, Brandon MP, Zhou Z-Y, Lin J-L, Hardacre C, et al. Tetrahexahedral Pt nanocrystal catalysts decorated with ru adatoms and their enhanced activity in methanol electrooxidation. ACS Catal. 2012;2:708-15

[213] Huang L, Zhang X, Wang Q, Han Y, Fang Y, Dong S. Shape-control of Pt-ru nanocrystals: tuning surface structure for enhanced electrocatalytic methanol oxidation. J Am Chem Soc. 2018;140:1142-7.

[214] Chen X, Cai Z, Chen X, Oyama M. Synthesis of bimetallic PtPd nanocubes on graphene with N,N-dimethylformamide and their direct use for methanol electrocatalytic oxidation. Carbon. 2014;66:387-94.

[215] Yin AX, Min XQ, Zhang YW, Yan CH. Shape-selective synthesis and facet-dependent enhanced electrocatalytic activity and durability of monodisperse Sub-10 nm Pt-Pd tetrahedrons and cubes. J Am Chem Soc. 2011;133:3816-9. 
[216] Lee Y-W, Ko AR, Han S-B, Kim H-S, Park K-W. Synthesis of octahedral Pt-Pd alloy nanoparticles for improved catalytic activity and stability in methanol electrooxidation. Phys Chem Chem Phys. 2011;13:5569-72.

[217] Yin A-X, Min X-Q, Zhu W, Wu H-S, Zhang Y-W, Yan C-H. Multiply twinned Pt-Pd nanoicosahedrons as highly active electrocatalysts for methanol oxidation. Chem Commun. 2012;48:543-5.

[218] Zhan F, Bian T, Zhao W, Zhang H, Jin M, Yang D. Facile synthesis of Pd-Pt alloy concave nanocubes with high-index facets as electrocatalysts for methanol oxidation. Cryst Eng Comm. 2014;16:2411-6.

[219] Zhang ], Xi C, Feng C, Xia H, Wang D, Tao X. High yield seedless synthesis of high-quality gold nanocrystals with various shapes. Langmuir. 2014;30:2480-9.

[220] Venkatasubramanian R, He ], Johnson MW, Stern I, Kim DH, Pesika NS. Additive-mediated electrochemical synthesis of platelike copper crystals for methanol electrooxidation. Langmuir. 2013;29:13135-9.

[221] Qi Y, Bian T, Choi S-I, Jiang Y, Jin C, Fu M, et al. Kinetically controlled synthesis of Pt-Cu alloy concave nanocubes with high-index facets for methanol electro-oxidation. Chem Commun. 2014;50:560-2.

[222] Yin A-X, Min X-Q, Zhu W, Liu W-C, Zhang Y-W, Yan C-H. Pt-Cu and Pt-Pd-Cu concave nanocubes with high-index facets and superior electrocatalytic activity. Chem - Eur ]. 2012;18:777-82.

[223] Luo X, Liu Y, Zhang H, Yang B. Shape-selective synthesis and facet-dependent electrocatalytic activity of CoPt3 nanocrystals. Cryst Eng Comm. 2012;14:3359-62.

[224] Kang Y, Pyo ]B, Ye X, Gordon TR, Murray CB. Synthesis, shape control, and methanol electro-oxidation properties of Pt-Zn alloy and Pt 3Zn intermetallic nanocrystals. ACS Nano. 2012;6:5642-7.

[225] Zhang N, Bu L, Guo S, Guo ], Huang X. Screw thread-like platinum-copper nanowires bounded with high-index facets for efficient electrocatalysis. Nano Lett. 2016;16:5037-43.

[226] Du H, Luo S, Wang K, Tang M, Sriphathoorat R, Jin Y, et al. High-quality and deeply excavated $\mathrm{Pt}_{3}$ Co nanocubes as efficient catalysts for liquid fuel electrooxidation. Chem Mater. 2017;29:9613-7.

[227] Chen Q, Cao Z, Du G, Kuang Q, Huang ], Xie Z, et al. Excavated octahedral Pt-Co alloy nanocrystals built with ultrathin nanosheets as superior multifunctional electrocatalysts for energy conversion applications. Nano Energy. 2017;39:582-9.

[228] Sun X, Huang B, Cui X, Feng BE, Huang X. Platinum-copper rhombic dodecahedral nanoframes with tunable channels as efficient bifunctional electrocatalysts for fuel-cell reactions. Chem Cat Chem. 2018;10:931-5.

[229] Li ], Rong H, Tong X, Wang P, Chen T, Wang Z. Platinum-silver alloyed octahedral nanocrystals as electrocatalyst for methanol oxidation reaction. J Colloid Interface Sci. 2018;513:251-7.

[230] Tang ]-X, Chen Q-S, You L-X, Liao H-G, Sun S-G, Zhou S-C, et al. Screw-like PdPt nanowires as highly efficient electrocatalysts for methanol and ethylene glycol oxidation. ] Mater Chem. 2018;6:2327-36.

[231] Li C, Liu T, He T, Ni B, Yuan Q, Wang X. Composition-driven shape evolution to Cu-rich PtCu octahedral alloy nanocrystals as superior bifunctional catalysts for methanol oxidation and oxygen reduction reaction. Nanoscale. 2018;10:4670-4.

[232] Colmati F, Tremiliosi-Filho G, Conzalez ER, Berná A, Herrero E, Feliu JM. Surface structure effects on the electrochemical oxidation of ethanol on platinum single crystal electrodes. Faraday Discuss. 2009;140:379-97.

[233] Koper MT, Lai SC, Herrero E. Mechanisms of the oxidation of carbon monoxide and small organic molecules at metal electrodes in fuel cell catalysis, a surface science approach. Fuel Cell Catalysis: A Surface Science Approach. In: Koper MT, editor. John Wiley \& Sons, 2009:159-208.

[234] Busó-Rogero C, Herrero E, Feliu JM. Ethanol oxidation on pt single-crystal electrodes: surface-structure effects in alkaline medium. Chem Phys Chem. 2014;15:2019-28.

[235] Wei L, Zhou ZY, Chen SP, Xu CD, Su D, Schuster ME, et al. Electrochemically shape-controlled synthesis in deep eutectic solvents: triambic icosahedral platinum nanocrystals with high-index facets and their enhanced catalytic activity. Chem Commun. 2013;49:11152-4.

[236] Zhang L, Chen D, Jiang Z, Zhang ], Xie S, Kuang Q, et al. Facile syntheses and enhanced electrocatalytic activities of Pt nanocrystals with $\{$ hkk\} high-index surfaces. Nano Res. 2012;5:181-9.

[237] Zhou ZY, Shang S], Tian N, Wu BH, Zheng NF, Xu BB, et al. Shape transformation from Pt nanocubes to tetrahexahedra with size near 10 $\mathrm{nm}$. Electrochem commun. 2012;22:61-4.

[238] Buso-Rogero C, Crozovski V, Vidal-Iglesias F], Solla-Cullon ], Herrero E, Feliu JM. Surface structure and anion effects in the oxidation of ethanol on platinum nanoparticles. ] Mater Chem. 2013;1:7068-76.

[239] Busó-Rogero C, Solla-Gullón ], Vidal-Iglesias F], Herrero E, Feliu JM. Oxidation of ethanol on platinum nanoparticles: surface structure and aggregation effects in alkaline medium. J Solid State Electrochem. 2016;20:1095-106.

[240] Busó-Rogero C, Brimaud S, Solla-Cullón ], Vidal-Iglesias F], Herrero E, Behm R], et al. Ethanol oxidation on shape-controlled platinum nanoparticles at different $\mathrm{pHs}$ : A combined in situ IR spectroscopy and online mass spectrometry study. J Electroanalytical Chem. 2016;763:116-24.

[241] Zhang ], Zhang L, Xie S, Kuang Q, Han X, Xie Z, et al. Synthesis of concave palladium nanocubes with high-index surfaces and high electrocatalytic activities. Chem Eur ]. 2011;17:9915-9.

[242] Yu N-F, Tian N, Zhou Z-Y, Huang L, Xiao ], Wen Y-H, et al. Electrochemical synthesis of tetrahexahedral rhodium nanocrystals with extraordinarily high surface energy and high electrocatalytic activity. Angew Chem Int Ed. 2014;53:5097-101.

[243] Chen X, Cai Z, Chen X, Oyama M. Green synthesis of graphene-PtPd alloy nanoparticles with high electrocatalytic performance for ethanol oxidation. ] Mater Chem. 2014;2:315-20.

[244] Rao L, Jiang Y-X, Zhang B-W, Cai Y-R, Sun S-G. High activity of cubic PtRh alloys supported on graphene towards ethanol electrooxidation. Phys Chem Chem Phys. 2014;16:13662-71.

[245] Hong JW, Lee YW, Kim M, Kang SW, Han SW. One-pot synthesis and electrocatalytic activity of octapodal Au-Pd nanoparticles. Chem Commun. 2011;47:2553-5. (Cambridge, United Kingdom).

[246] Zhang ], Hou C, Huang H, Zhang L, Jiang Z, Chen G, et al. Surfactant-concentration-dependent shape evolution of Au-pd alloy nanocrystals from rhombic dodecahedron to trisoctahedron and hexoctahedron. Small. 2013;9:538-44. 
[247] Rizo R, Arán-Ais RM, Padgett E, Muller DA, Lázaro M], Solla-Cullón ], et al. Pt-richcore/Sn-richsubsurface/Ptskin nanocubes as highly active and stable electrocatalysts for the ethanol oxidation reaction. J Am Chem Soc. 2018;140:3791-7.

[248] Erini N, Beermann V, Cocyla M, Cliech M, Heggen M, Dunin-Borkowski RE, et al. The effect of surface site ensembles on the activity and selectivity of ethanol electrooxidation by octahedral PtNiRh nanoparticles. Angew Chem Int Ed. 2017;56:6533-8.

[249] Wang P, Lin X, Yang B, Jin JM, Hardacre C, Yu NF, et al. Activity enhancement of tetrahexahedral Pd nanocrystals by Bi decoration towards ethanol electrooxidation in alkaline media. Electrochim Acta. 2015;162:290-9.

[250] Busó-Rogero C, Solla-Cullón ], Vidal-Iglesias F], Herrero E, Feliu JM. Adatom modified shape-controlled platinum nanoparticles towards ethanol oxidation. Electrochim Acta. 2016;196:270-9.

[251] Xing W, Yin G, Zhang ]. Rotating electrode methods and oxygen reduction electrocatalysts. Elsevier Science, 2014.

[252] Katsounaros I, Cherevko S, Zeradjanin AR, Mayrhofer K]. Oxygen electrochemistry as a cornerstone for sustainable energy conversion. Angew Chem Int Ed. 2014;53:102-21.

[253] Guo S, Zhang S, Sun S. Tuning nanoparticle catalysis for the oxygen reduction reaction. Angew Chem Int Ed. 2013;52:8526-44.

[254] Lee ], Jeong B, Ocon JD. Oxygen electrocatalysis in chemical energy conversion and storage technologies. Curr Appl Phys. 2013;13:30921.

[255] Wu ], Yang H. Platinum-based oxygen reduction electrocatalysts. Acc Chem Res. 2013;46:1848-57.

[256] Maciá MD, Campina JM, Herrero E, Feliu JM. On the kinetics of oxygen reduction on platinum stepped surfaces in acidic media. ] Electroanalytical Chem. 2004;564:141-50.

[257] Kuzume A, Herrero E, Feliu JM. Oxygen reduction on stepped platinum surfaces in acidic media.] Electroanalytical Chem. 2007;599:333-43.

[258] Cómez-Marín AM, Rizo R, Feliu JM. Oxygen reduction reaction at Pt single crystals: a critical overview. Catalysis Sci Technol. 2014;4:1685-98

[259] Bandarenka AS, Hansen HA, Rossmeis I], Stephens IE. Elucidating the activity of stepped Pt single crystals for oxygen reduction. Phys Chem Chem Phys. 2014;16:13625-9.

[260] Briega-Martos V, Herrero E, Feliu ]M. Effect of pH and water structure on the oxygen reduction reaction on platinum electrodes. Electrochim Acta. 2017:241:497-509.

[261] Inaba M, Ando M, Hatanaka A, Nomoto A, Matsuzawa K, Tasaka A, et al. Controlled growth and shape formation of platinum nanoparticles and their electrochemical properties. Electrochim Acta. 2006;52:1632-8.

[262] Sanchez-Sanchez CM, Solla-Gullon ], Vidal-Iglesias F], Aldaz A, Montiel V, Herrero E. Imaging structure sensitive catalysis on different shape-controlled platinum nanoparticles. ] Am Chem Soc. 2010;132:5622-4.

[263] Tripković V, Cerri I, Bligaard T, RossmeisI ]. The influence of particle shape and size on the activity of platinum nanoparticles for oxygen reduction reaction: A density functional theory study. Catal Lett. 2014;144:380-8.

[264] Xiao L, Zhuang L, Liu Y, Lu ], Abruña HD. Activating Pd by morphology tailoring for oxygen reduction. ] Am Chem Soc. 2008;131:602-8.

[265] Shao M, Yu T, Odell JH, Jin M, Xia Y. Structural dependence of oxygen reduction reaction on palladium nanocrystals. Chem Commun. 2011;47:6566-8.

[266] Lee C-L, Chiou H-P, Liu C-R. Palladium nanocubes enclosed by (100) planes as electrocatalyst for alkaline oxygen electroreduction. Int ] Hydrogen Energy. 2012;37:3993-7.

[267] Zhang H, Jin M, Xiong Y, Lim B, Xia Y. Shape-controlled synthesis of Pd nanocrystals and their catalytic applications. Acc Chem Res. 2012;46:1783-94.

[268] Shao M. Palladium-based electrocatalysts for oxygen reduction reaction in electrocatalysis in fuel cells, vol. 9. Lecture Notes in Energy Shao M. editor. Springer, 2013:513-31.

[269] Lim B, Jiang M, Camargo PH, Cho EC, Tao ], Lu X, et al. Pd-Pt bimetallic nanodendrites with high activity for oxygen reduction. Science. 2009;324:1302-5.

[270] Oezaslan M, Hasché F, Strasser P. Pt-based core-shell catalyst architectures for oxygen fuel cell electrodes.] Phys Chem Lett. 2013;4:3273-91.

[271] Can L, Cui C, Rudi S, Strasser P. Core-shell and nanoporous particle architectures and their effect on the activity and stability of Pt ORR electrocatalysts. Top Catal. 2014;57:236-44.

[272] Li D, Wang C, Strmcnik DS, Tripkovic DV, Sun X, Kang Y, et al. Functional links between Pt single crystal morphology and nanoparticles with different size and shape: the oxygen reduction reaction case. Energy Environ Sci. 2014.

[273] Stamenkovic VR, Fowler B, Mun BS, Wang CF, Ross PN, Lucas CA, et al. Improved oxygen reduction activity on Pt ${ }_{3} \mathrm{Ni}(111)$ via increased surface site availability. Science. 2007;315:493-7.

[274] Casteiger HA, Markovic NM. Just a dream or future reality? Science. 2009;324:48-9.

[275] Zhang ], Yang H, Fang ], Zou S. Synthesis and oxygen reduction activity of shape-controlled Pt ${ }_{3}$ Ni nanopolyhedra. Nano Lett. 2010;10:638-44.

[276] Strasser P. Catalysts by platonic design. Science. 2015;349:379-80.

[277] Mistry H, Varela AS, Kühl S, Strasser P, Cuenya BR. Nanostructured electrocatalysts with tunable activity and selectivity. Nat Rev Mater. 2016;1. Article number: 16009. DOI: 10.1038/natrevmats.2016.9.

[278] Hori Y, Murata A, Takahashi R. Formation of hydrocarbons in the electrochemical reduction of carbon dioxide at a copper electrode in aqueous solution. J Chem Soc, Faraday Trans I: Phys Chem Condens Phases. 1989;85:2309-26.

[279] Kortlever R, Shen ], Schouten K], Calle-Vallejo F, Koper MT. Catalysts and reaction pathways for the electrochemical reduction of carbon dioxide. J Phys Chem Lett. 2015;6:4073-82.

[280] Gupta N, Cattrell M, Macdougall B. Calculation for the cathode surface concentrations in the electrochemical reduction of $\mathrm{CO}_{2}$ in $\mathrm{KHCO}_{3}$ solutions. J Appl Electrochemistry. 2006;36:161-72.

[281] Varela AS, Ju W, Reier T, Strasser P. Tuning the catalytic activity and selectivity of $\mathrm{Cu}$ for $\mathrm{CO}_{2}$ eletroreduction in the presence of halides. ACS Catal. 2016;6:2136-44. 
[282] Varela AS, Kroschel M, Reier T, Strasser P. Controlling the selectivity of $\mathrm{CO}_{2}$ electroreduction on copper: the effect of the electrolyte concentration and the importance of the local pH. Catalysis Today. 2016;260:8-13.

[283] Azuma M, Hashimoto K, Hiramoto M, Watanabeand M, Sakata T. Electrochemical reduction of carbon dioxide on various metal electrodes in low-temperature aqueous $\mathrm{KHCO}_{3}$ media. J Electrochem Soc. 1990;137:1772-8.

[284] Hori Y, Wakebe H, Tsukamoto T, Koga O. Electrocatalytic process of $\mathrm{CO}$ selectivity in electrochemical reduction of $\mathrm{CO}_{2}$ at metal electrodes in aqueous media. Electrochim Acta. 1994;39:1833-9.

[285] Hori Y. Electrochemical $\mathrm{CO}_{2}$ reduction on metal electrodes. In: Modern aspects of electrochemistry, vol. 42. New York: Springer, 2008:89-189.

[286] Durand W], Peterson AA, Studt F, Abild-Pedersen F, Nørskov ]K. Structure effects on the energetics of the electrochemical reduction of $\mathrm{CO}_{2}$ by copper surfaces. Surf Sci. 2011;605(15-16:1354-9.

[287] Kuhl KP, Hatsukade T, Cave ER, Abram DN, Kibsgaard ], Jaramillo TF. Electrocatalytic conversion of carbon dioxide to methane and methanol on transition metal surfaces. ] Am Chem Soc. 2014;136:14107-13.

[288] Qiao ], Liu Y, Zhan ]. Electrochemical reduction of carbon dioxide: fundamentals and technologies. CRC Press, Taylor and Francis Croup, 2016.

[289] Kuhl KP, Cave ER, Abram DN, Jaramillo TF. New insights into the electrochemical reduction of carbon dioxide on metallic copper surfaces. Energy Environ Sci. 2012;5:7050-9.

[290] Reske R, Mistry H, Behafarid F, Cuenya BR, Strasser P. Particle size effects in the catalytic electroreduction of $\mathrm{CO}_{2}$ on Cu nanoparticles. ] Am Chem Soc. 2014;136:6978-86.

[291] Kas R, Kortlever R, Milbrat A, Koper MT, Mul G, Baltrusaitis ]. Electrochemical $\mathrm{CO}_{2}$ reduction on $\mathrm{Cu}_{2} \mathrm{O}$-derived copper nanoparticles: controlling the catalytic selectivity of hydrocarbons. Phys Chem Chem Phys. 2014;16:12194-201.

[292] Huang Y, Handoko AD, Hirunsit P, Yeo BS. Electrochemical reduction of $\mathrm{CO}_{2}$ using copper single-crystal surfaces: effects of $\mathrm{CO}^{*}$ coverage on the selective formation of ethylene. ACS Catal. 2017;7:1749-1756.

[293] Chen CS, Handoko AD, Wan JH, Ma L, Ren D, Yeo BS. Stable and selective electrochemical reduction of carbon dioxide to ethylene on copper mesocrystals. Catal Sci Technol. 2015;5:161-8.

[294] Roberts FS, Kuhl KP, Nilsson A. High selectivity for ethylene from carbon dioxide reduction over copper nanocube electrocatalysts. Angew Chem Int Ed. 2015;54:5179-82.

[295] Eilert A, Roberts FS, Friebel D, Nilsson A. Formation of copper catalysts for $\mathrm{CO}_{2}$ reduction with high ethylene/methane product ratio investigated with in situ $X$ ray absorption spectroscopy. ] Phys Chem Lett. 2016;7:1466-70.

[296] Kwon Y, Lum Y, Clark EL, Ager JW, Bell AT. CO ${ }_{2}$ electroreduction with enhanced ethylene and ethanol selectivity by nanostructuring polycrystalline copper. Chem Electro Chem. 2016;3:1012-9.

[297] Gao D, Zegkinoglou I, Divins N], Scholten F, Sinev I, Grosse P, et al. Plasma-activated copper nanocube catalysts for efficient carbon dioxide electroreduction to hydrocarbons and alcohols. ACS Nano. 2017;11:4825-31.

[298] Loiudice A, Lobaccaro P, Kamali EA, Thao T, Huang BH, Ager JW, et al. Tailoring copper nanocrystals towards C2 products in electrochemical $\mathrm{CO}_{2}$ reduction. Angew Chem Int Ed. 2016;55:5789-92.

[299] Jeon HS, Kunze S, Scholten F, Cuenya BR. Prism-shaped Cu nanocatalysts for electrochemical $\mathrm{CO}_{2}$ reduction to ethylene. ACS Catal. 2018;8:531-5.

[300] Ma M, Djanashvili K, Smith WA. Selective electrochemical reduction of $\mathrm{CO}_{2}$ to $\mathrm{CO}$ on CuO-derived Cu nanowires. Phys Chem Chem Phys. 2015;17:20861-7.

[301] Raciti D, Livi K], Wang C. Highly dense Cu nanowires for low-overpotential $\mathrm{CO}_{2}$ reduction. NanoLett. 2015;15:6829-35.

[302] Ma M, Djanashvili K, Smith WA. Controllable hydrocarbon formation from the electrochemical reduction of $\mathrm{CO}_{2}$ over $\mathrm{Cu}$ nanowire arrays. 2016;55:6680-4

[303] Chung ], Won DH, Koh ], Kim E-H, Woo SI. Hierarchical Cu pillar electrodes for electrochemical $\mathrm{CO}_{2}$ reduction to formic acid with low overpotential. Phys Chem Chem Phys. 2016;18:6252-8.

[304] Sen S, Liu D, Palmore GT. Electrochemical reduction of $\mathrm{CO}_{2}$ at copper nanofoams. ACS Catal. 2014;4:3091-5.

[305] Liu S, Tao H, Zeng L, Liu Q, Xu Z, Liu Q, et al. Shape-dependent electrocatalytic reduction of $\mathrm{CO}_{2}$ to $\mathrm{CO}$ on triangular silver nanoplates. ] Am Chem Soc. 2017;139:2160-3.

[306] Peng X, Karakalos SC, Mustain WE. Preferentially oriented Ag nanocrystals with extremely high activity and faradaic efficiency for $\mathrm{CO}_{2}$ electrochemical reduction to CO. ACS Appl Mater Interfaces. 2018;10:1734-42.

[307] Hsieh Y-C, Senanayake SD, Zhang Y, Xu W, Polyansky DE. Effect of chloride anions on the synthesis and enhanced catalytic activity of silver nanocoral electrodes for $\mathrm{CO}_{2}$ electroreduction. ACS Catal. 2015;5:5349-56.

[308] Lu Q, Rosen ], Zhou Y, Hutchings CS, Kimmel YC, Chen JC, et al. A selective and efficient electrocatalyst for carbon dioxide reduction. Nat Commun. 2014;5:3242.

[309] Klinkova A, De Luna P, Dinh C-T, Voznyy O, Larin EM, Kumacheva E, et al. Rational design of efficient palladium catalysts for electroreduction of carbon dioxide to formate. ACS Catal. 2016;6:8115-20.

[310] Gao D, Zhou H, Cai F, Wang ], Wang G, Bao X. Pd-Containing nanostructures for electrochemical $\mathrm{CO}_{2}$ reduction reaction. ACS Catal. 2018;8:1510-9.

[311] Huang H, Jia H, Liu Z, Gao P, Zhao ], Luo Z, et al. Understanding of strain effects in the electrochemical reduction of $\mathrm{CO}_{2}$ : using Pd Nanostructures as an Ideal Platform. Angew Chem Int Ed. 2017;56:3594-8.

[312] Rosen ], Hutchings CS, Lu Q, Forest RV, Moore A, Jiao F. Electrodeposited Zn dendrites with enhanced CO selectivity for electrocatalytic $\mathrm{CO}_{2}$ reduction. ACS Catal. 2015;5:4586-91.

[313] Won DH, Shin H, Koh ], Chung ], Lee HS, Kim H, et al. Highly efficient, selective, and stable $\mathrm{CO}_{2}$ electroreduction on a hexagonal Zn catalyst. Angew Chem Int Ed. 2016;55:9297-300.

[314] Zhong H, Qiu Y, Zhang T, Li X, Zhang H, Chen X. Bismuth nanodendrites as a high performance electrocatalyst for selective conversion of $\mathrm{CO}_{2}$ to formate. ] Mater Chem. 2016;4:13746-53. 
[315] Koh JH, Won DH, Eom T, Kim N-K, Jung KD, Kim H, et al. Facile $\mathrm{CO}_{2}$ electro-reduction to formate via oxygen bidentate intermediate stabilized by high-index planes of Bi dendrite catalyst. ACS Catal. 2017;7:5071-7.

[316] Kim S, Dong W], Gim S, Sohn W, Park JY, Yoo C], et al. Shape-controlled bismuth nanoflakes as highly selective catalysts for electrochemical carbon dioxide reduction to formate. 2017;39:44-52.

[317] Zhang Z, Chi M, Veith CM, Zhang P, Lutterman DA, Rosenthal ], et al. Rational design of Bi nanoparticles for efficient electrochemical $\mathrm{CO}_{2}$ reduction: the elucidation of size and surface condition effects. ACS Catal. 2016;6:6255-64.

[318] Zhu W, Zhang Y-J, Zhang H, Lv H, Li Q, Michalsky R, et al. Active and selective conversion of $\mathrm{CO}_{2}$ to CO on ultrathin Au nanowires. ] Am Chem Soc. 2014;136:16132-5.

[319] Back S, Yeom MS, ]ung Y. Understanding the effects of Au morphology on $\mathrm{CO}_{2}$ electrocatalysis. ] Phys Chem C. 2018;122:4274-80.

[320] Lee H-E, Yang KD, Yoon SM, Ahn H-Y, Lee YY, Chang H, et al. Concave rhombic dodecahedral Au nanocatalyst with multiple high-index facets for $\mathrm{CO}_{2}$ reduction. ACS Nano. 2015;9:8384-93.

[321] Kim ], Song JT, Ryoo H, Kim ]-G, Chung S-Y, Oh ]. Morphology-controlled Au nanostructures for efficient and selective electrochemical $\mathrm{CO}_{2}$ reduction. J Mater Chem. 2018;6:5119-28.

[322] Monzó ], Malewski Y, Kortlever R, Vidal-Iglesias F], Solla-Cullón ], Koper MT, et al. Enhanced electrocatalytic activity of Au@Cu core@shell nanoparticles towards $\mathrm{CO}_{2}$ reduction. J Mater Chem. 2015;3:23690-8.

[323] Zhao X, Luo B, Long R, Wang C, Xiong Y. Composition-dependent activity of Cu-pt alloy nanocubes for electrocatalytic $\mathrm{CO}_{2}$ reduction. ] Mater Chem. 2015;3:4134-8.

[324] Zhang F-Y, Sheng T, Tian N, Liu L, Xiao C, Lu B-A, et al. Cu overlayers on tetrahexahedral Pd nanocrystals with high-index facets for $\mathrm{CO}_{2}$ electroreduction to alcohols. Chem Commun. 2017;53:8085-8.

[325] Zhang L, Zhao Z-J, Gong ]. Nanostructured materials for heterogeneous electrocatalytic $\mathrm{CO}_{2}$ reduction and related reaction mechanisms. Angew Chem Int Ed. 2017;56:11326-53.

[326] Zhang W, Hu Y, Ma L, Zhu G, Wang Y, Xue X, et al. Progress and perspective of electrocatalytic $\mathrm{CO}_{2}$ reduction for renewable carbonaceous fuels and chemicals. Adv Sci. 2018;5:1700275-99.

[327] Yang W, Dastafkan K, Jia C, Zhao C. Design of electrocatalyst and electrochemical cells for carbon dioxide reduction reactions. Adv Mate Technol. 2018;1700377-97.

[328] Sahu SC, Hayes AW. Toxicity of nanomaterials found in human environment: A literature review. Toxicol Res Appl. 2017;1:1-13.

[329] Handy RD, Shaw B]. Toxic effects of nanoparticles and nanomaterials: implications for public health, risk assessment and the public perception of nanotechnology. ] Health, Risk Soc. 2007;9:125-44.

[330] Buzea C, Pacheco II, Robbie K. Nanomaterials and nanoparticles: sources and toxicity. Biointerphases. 2007;2:MR17-MR71.

[331] Kaphle A, Navya PN, Umapathi A, Daima HK. Nanomaterials for agriculture, food and environment: applications, toxicity and regulation. Environ Chem Lett. 2018;16:43-58.

[332] Lee ], Mahendra S, Alvarez P]. Nanomaterials in the construction industry: a review of their applications and environmental health and safety considerations. ACS Nano. 2010;4:3580-90.

[333] Benson JM. Safety considerations when handling metal powders. ] South Afr Inst Mining Metallurgy. 2012;112:563-75.

[334] Bussy C, Ali-Boucetta H, Kostarelos K. Safety considerations for graphene: lessons learnt from carbon nanotubes. Acc Chem Res. 2013;46:692-701.

[335] Park MV, Bleeker EA, Brand W, Cassee FR, Van Elk M, Cosens I, et al. Considerations for safe innovation: the case of graphene. ACS Nano. 2017;11:9574-93.

[336] Al-Mubaddel FS, Haider S, Al-Masry WA, Al-Zeghayer Y, Imran M, Haider A, et al. Engineered nanostructure: a review of their synthesis, characterization and toxic hazard considerations. Arabian ] Chem. 2017;10:S376-S388.

[337] Jeevanandam ], Barhoum A, Chan YS, Dufresne A, Danquah MK. Review on nanoparticles and nanostructured materials: history, sources, toxicity and regulations. Beilstein ] Nanotechnol. 2018;9:1050-74.

[338] Lee CC, Mackay JA, Fréchet JM, Szoka FC. Designing dendrimers for biological applications. Nat Biotechnol. 2005;23:1517-26.

[339] Carlson C, Hussain SM, Schrand AM, Braydich-Stolle LK, Hess KL, Jones RL, et al. Unique cellular interaction of silver nanoparticles: sizedependent generation of reactive oxygen species. J Phys Chem B. 2008;112:13608-19.

[340] El Badawy AM, Silva RC, Morris B, Scheckel KC, Suidan MT, Tolaymat TM. Surface charge-dependent toxicity of silver nanoparticles. Environ Sci Technol. 2011;45:283-7.

[341] Albanese A, Tang PS, Chan WC. The effect of nanoparticle size, shape, and surface chemistry on biological systems. Annu Rev Biomed Eng. 2012;14:1-16.

[342] Daima HK, Towards fine-tuning the surface corona of inorganic and organic nanomaterials to control their properties at nano-bio interface, 2013, PhD thesis, School of Applied Sciences RMIT.

[343] Daima HK, Selvakannan PR, Shukla R, Bhargava SK, Bansal V. Fine-tuning the antimicrobial profile of biocompatible gold nanoparticles by sequential surface functionalization using polyoxometalates and lysine. PLoS One. 2013;8:e79676-90.

[344] Chen LQ, Fang L, Ling ], Ding CZ, Kang B, Huang CZ. Nanotoxicity of silver nanoparticles to red blood cells: size-dependent adsorption, uptake, and hemolytic activity. Chem Res Toxicol. 2015;28:501-9.

[345] Navya PN, Daima HK. Rational engineering of physicochemical properties of nanomaterials for biomedical applications with nanotoxicological perspectives. Nano Convergence. 2016;3:1-14.

\section{Bionotes}




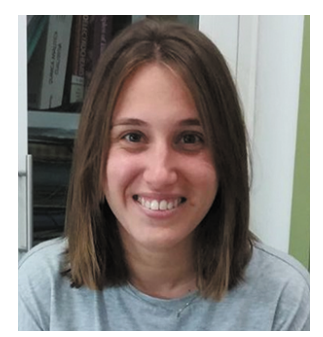

Leticia García Cruz received her Ph.D. from University of Alicante in 2016. She is currently senior research in Institute of Electrochemistry of University of Alicante. She has over 7 years of experience in the field of development of electrocatalysts, nanoparticle synthesis, and carbon materials, for electrochemical oxidation process. Her research covers manufacture of gas diffusion electrodes, design of electrochemical reactors and optimization of electrochemical process, and ion-exchange membrane synthesis. More recently, her research is focused on the development of electrocatalysts for electrochemical reduction of $\mathrm{CO}_{2}$.

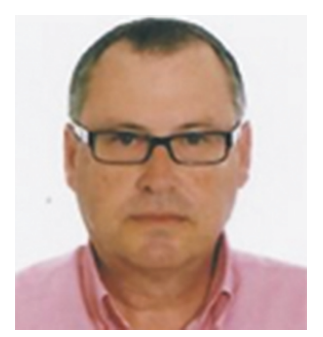

Vicente Montiel Leguey is full professor of Physical Chemistry at the University of Alicante and current director of the Institute of Electrochemistry. His research is mainly focused on Applied Electrochemistry (electrosynthesis; wastewater treatment by electrochemical methods without and with photovoltaic energy coupling; sensors and biosensors; preparation, characterization, and use of nanoparticulated materials; and design and optimization of redox flow batteries). His experience covers the development of different electrochemical processes, from laboratory scale to pre-industrial pilot plant. Several processes have been transferred to the industry. He was President of the Electrochemistry Group of the Spanish Society of Chemistry (since 2012 to 2016). Currently, he is President of the Division of Chemistry at Alicante territory.

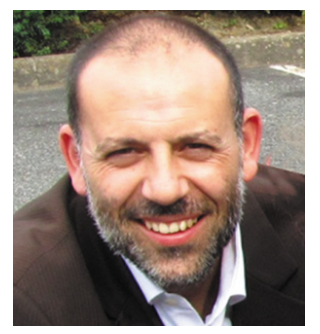

Jose Solla Gullón studied at the Universidad de Santiago de Compostela, and carried out his PhD at the Universidad de Alicante (awarded in 2003). Currently, he is senior researcher in the Institute of Electrochemistry at the University of Alicante. His research is focused on the synthesis, characterization, and electrocatalytic properties of size- and shape-controlled metal nanoparticles. 